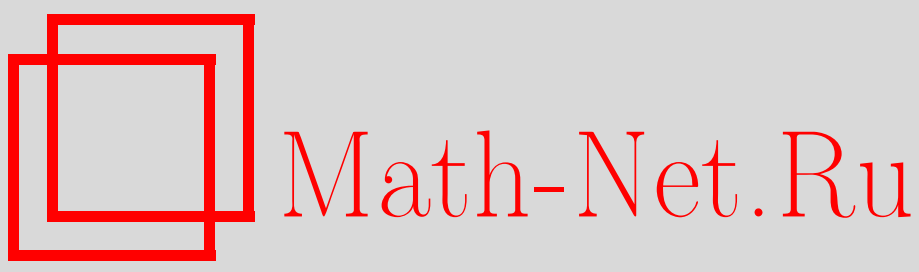

А. Ю. Ольшанский, Об искривлении подгрупп конечно-определенных групп, $M a$ тем. сб., 1997, том 188, номер 11, 51-98

DOI: https://doi.org/10.4213/sm276

Использование Общероссийского математического портала Math-Net.Ru подразумевает, что вы прочитали и согласны с пользовательским соглашением

http://www.mathnet.ru/rus/agreement

Параметры загрузки:

IP : 52.90 .164 .192

26 апреля 2023 г., $17: 24: 42$ 
УДК 512

\title{
А.Ю. Ольшанский
}

\section{Об искривлении подгрупп конечно-определенных групп}

\begin{abstract}
Доказано, что всякая вычислимая функция $G \rightarrow \mathbb{N}=\{0,1, \ldots\}$ на группе $G$ (с некоторыми необходимыми ограничениями) может быть с точностью до эквивалентности реализована как функция длины элементов посредством вложения группы $G$ в подходящую конечно-определенную группу. Например, длина степени $g^{n}$ элемента $g$ конечно-определенной групшы может расти, как $n \theta$, для любого вычислимого числа $\theta \in(0,1]$. Тем самьм дается ответ на вопрос М. Громова. Основньм инструментом является доказанньй в статье усиленный вариант вложения Хигмэна, при котором сохраняются длины элементов.

Библиографрия: 10 названий.
\end{abstract}

\section{§1. Введение}

В группе $G$ с конечным множеством порождающих $\mathscr{A}=\left\{a_{1}, \ldots, a_{m}\right\}$ каждый элемент $g$ имеет длину $|g|=|g|_{\mathscr{A}}$, равную длине записи $\|W\|$ кратчайшего слова $W$ в алфавите $\mathscr{A}^{ \pm 1}$, представляюшего элемент $g$. При изоморфном вложении группы $G$ в другую группу $H$ с системой порождающих $\mathscr{B}=\left\{b_{1}, \ldots, b_{n}\right\}$ справедливо очевидное неравенство

$$
|g|_{\mathscr{B}} \leqslant c|g|_{\mathscr{A}}
$$

где константа $c=\max \left\{\left|a_{1}\right|_{\mathscr{B}}, \ldots,\left|a_{m}\right|_{\mathscr{B}}\right\}$ не зависит от выбора элемента $g$. В частности, для двух систем порождающих $\mathscr{A}$ и $\mathscr{B}$ одной группы $G$ можно подобрать положительные константы $c_{1}$ и $c_{2}$, такие что для любого $g \in G$

$$
c_{1}|g|_{\mathscr{A}} \leqslant|g|_{\mathscr{B}} \leqslant c_{2}|g|_{\mathscr{A}} .
$$

В статье [1] дан ответ на следующий вопрос. Какие функции $l: G \rightarrow \mathbb{N}=$ $\{0,1,2, \ldots\}$ могут быть реализованы с точностью до эквивалентности для данной счетной группы $G$ как функции длины $g \mapsto|g|_{\mathscr{B}}$ при вложении группы $G$ в некоторую групту $H$ с конечной системой порождающих $\mathscr{B}$. Понятие эквивалентности обусловлено при этом неравенствами (1.2), а именно, две функции $l_{1}, l_{2}: G \rightarrow \mathbb{N}$ называются әквивалентными, если сушествуют положительные константы $c_{1}, c_{2}$ такие, что

$$
c_{1} l_{1}(g) \leqslant l_{2}(g) \leqslant c_{2} l_{1}(g)
$$

для любого элемента $g \in G$.

В следующей теореме автора нетривиальной является “достаточность”.

Работа вьполнена при частичной поддержке Российского фонда фундаментальных исследований (грант № 96-01-420). 
Tеорема 1 [1]. Пусть $l: G \rightarrow \mathbb{N}$ - функиия, определенная вложением группь $G$ в некоторую группу $R$ с конечной системой порождающих $\mathscr{B}=$ $\left\{b_{1}, \ldots, b_{n}\right\}$, m.e. $l(g)=|g|_{\mathscr{B}}$. Тогдa

(D1) $l(g)=l\left(g^{-1}\right)$ для каждого $g \in G$, и $l(g)=0$ в том и только в том случае, когда $g=1$

(D2) $l(g h) \leqslant l(g)+l(h) \partial \Omega я g, h \in G$;

(D3) существует полоэительное число а такое, что саrd $\{g \in G \mid l(g) \leqslant r\}$ $\leqslant a^{r}$ для любого $r \in \mathbb{N}$.

Наоборот, для любой группь $G$ и любой функции $l: G \rightarrow \mathbb{N}$, удовлетворяющей условиям (D1)-(D3) (коротко - D-условию), существует изоморфное вложсение группь $G$ в некоторую 2-порожденную группу $R$ с порождающим множеством $\mathscr{B}=\left\{b_{1}, b_{2}\right\}$ такое, что функиия $g \mapsto|g|_{\mathscr{B}}$ әквивалентна функuиul.

В частном случае бесконечной циклической группы $G=\langle g\rangle$ получаются, например, группы $R_{\alpha} \geqslant G$, в которых длина степени $g^{i}$ растет как $i^{\alpha}$ для любого заранее фиксированного $\alpha \in(0,1]$. Тем самьм дается ответ на соответствуюший вопрос Громова [2].

Однако разнообразные эффекты искривления циклических подгрупп встречаются уже в конечно-определенных группах и даже в группах с одним соотношением. K примеру, в группе $\langle a, b, c \mid[a, b]=c,[a, c]=[b, c]=1\rangle$ функция $\left|c^{i}\right|$ растет как $\sqrt{i}$, иначе говоря, центральная циклическая подгруппа $\langle c\rangle$ имеет квадратичное "искривление" (distortion). (За характеристику искривления подгруппы $G$ в группе $H$ принимается функция $\operatorname{disto}(r)=\max _{|g|_{\mathscr{B}} \leqslant r}|g|_{\mathscr{A}}$, хотя по ней и нельзя восстановить класс эквивалентности функции $g \mapsto|g|_{\mathscr{B}}$ на группе $G$.) В группе $\left\langle a, b \mid a b a^{-1}=b^{2}\right\rangle$ функция $\left|b^{i}\right|$ растет, как $\log i$, т.е. циклическая подгруппа $\langle b\rangle$ имеет экспоненциальное искривление. Пример более чем мультиэкспоненциального искривления и другие примеры можно найти в [3] и [2]. Серия примеров вложений для специально построенных нециклических групп в конечно-определенные группы с функциями disto $\sim r^{q}$ для всех рациональных показателей $q \geqslant 1$ построена в [2].

Поэтому в "проблеме реализации" Громов выделяет в [2] нахождение возможных искривлений циклических подгрупп в конечно-определенных группах. При таком ограничении "реализовать" любую функцию с D-условием, как в теореме 1 , заведомо не удастся, хотя бы по причине континуальности мощности множества классов эквивалентности функций с D-условием. (Все функции вида $i^{\alpha}$ попарно не эквивалентны.) Но все "разумно определенные" функции искривлений удается реализовать, причем не только для циклических подгрупп.

Теорема 2. Пусть на группе $G$ задана вычислимая функиия $l$ со свойствами (D1)-(D3). Тогда группу $G$ можно изоморфно вложить в некоторую конечно определенную группу $H$ так, что функция $l$ эквивалентна ограниче-

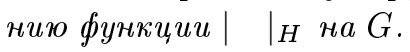

Здесь функция $l$ считается вычислимой, если по любому заданию какого-либо элемента $g$ словом относительно некоторой системы порождающих можно алгоритмически вычислить значение $l(g)$. При этом конечность числа порождающих 
в $G$ можно не предполагать. Далее, запись $\mid$ |н имеет смысл независимо от выбора конечной системы порождающих в $H$, если рассматривать функции с точностью до эквивалентности. Заметим еше очевидное: если $H$ - группа с алгоритмически разрешимой проблемой равенства, то длины ее элементов относительно любой конечной системы порождающих вычислимы.

Как и предполагалось в [1], ключом к доказательству оказалось следующее усиление теоремы Хигмэна, которое позволяет вывести теорему 2 из теоремы 1.

ТеОрема 3. Пусть группа $R$ имеет конечную систему порождающих $\mathscr{A} u$ некоторое рекурсивно перечислимое множество (определяющих) соотношений. Тогда существует такое изоморфное влохсение группьи $R$ в некоторую группу $H$ с конечной системой порождающих $\mathscr{B}$ и конечной системой определяюших соотношений, что $|g|_{\mathscr{A}}=|g|_{\mathscr{B}}$ для любого $g \in G$.

Отметим, что для полугрупп утверждение теоремы 3 доказано недавно Бирже $[5]$.

Не составляет большого труда несколько усилить формулировку теоремы 3 , выбрав универсальную группу $H$. С учетом леммы 2.2 эту группу можно взять и в теореме 2 независимо от $G$ в качестве вместилища всевозможных "вычислимых искривлений" конечно-порожденных подгрупп.

Tеорема 4. Существует группа $U$ с конечной системой порождающих $\mathscr{B}$ и конечным множеством определяюших соотношений такая, что для всякой группь $H$ с конечной системой порожсдающих $\mathscr{A}$ и рекурсивно перечислимым множеством определяющих соотношений имеется изоморфное вложение $H \hookrightarrow U$, при котором $|g|_{\mathscr{A}} \leqslant|g|_{\mathscr{B}}$ для всех $g \in H$.

План изложения следующий. В $\S 2$ напоминается конструкция из [1] с объяснением рекурсивности задания групшы $R$ в теореме 1 в случае вычислимой функции $l$. В $\S 3$ приводится ряд полезных свойств гиперболических плоских графов, т.е. плоских графов с достаточно высокими валентностями вершин.

В $\S 4$ выписываются соотношения группы $H$ из теоремы 3. Они являются модификацией приведенных в гл. 12 книги Ротмана [6], в которой доказательство Бриттона теоремы Новикова-Буна (с учетом позднейших улучшений, данных Буном, Коллинзом и Миллером III) сопровождается доказательством Андера [7] теоремы вложения Хигмэна. (Пользуясь случаем, автор благодарит И. Рипса и С.В. Иванова, обративших его внимание на доказательство Андера.)

Изменение состоит в удлинении по сравнению с [6] соотношения (4.10), что позволяет обосновать и использовать затем некоторые гиперболические свойства диаграмм ван Кампена над копредставлением из $§ 4$. Выгоду такого рода модификации впервые продемонстрировали Бирже, Рипс и Сапир [8] при конструировании групп с заданной функцией Дэна. Однако в отличие от [8] мы не меняем машины Тьюринга по сравнению с [6], в частности, она остается детерминистской.

В геометрических пояснениях гл. 12 книги [6] и, более систематично, в статье [8] используется то обстоятельство, что соотношения, характерные для HNN-pacширения и похожие на них, структурируют диаграммы ван Кампена, расслаивая их на различные "полосы". Соответствуюшая терминология приводится в 5 , а в $\S 6$ доказывается лемма о невозможности кратных пересечений полос опреде- 
ленных типов в диаграммах без “втулок", т.е. без клеток, отвечающих соотношениям (4.10).

В $\S 7$ приводятся необходимые геометрические сведения о минимальных диаграммах над модифицированной группой Новикова-Буна, например, почему две втулки имеют не более одной общей “спицы” .

В $\S 8$ добавляются уже и соотношения типа Андера. Хотя исследуемая группа является конечно определенной, оказывается, что для исключения в минимальных диаграммах полос, “сильно огибающих втулки”, нужно допустить в качестве определяющих бесконечно много соотношений, а минимальность диаграмм следует понимать с учетом градуировки, т.е. с учетом рангов клеток.

Бесконечность множества соотношений, временно включаемых в список определяющих, приходится допустить и в 9 для исключения в минимальных диаграммах пар втулок, связанных большим числом спиц.

Для доказательства теоремы 3 в $\S 10$ строится четыре промежуточные подгруппы между $R$ и $H$ и проводится ряд промежуточных сравнений длин элементов.

B $\S 11$ используются все основные леммы, в частности, важное свойство дизъюнктности “производных овалов" в гиперболических графах, с помощью которого оцениваются длины участков границы минимальной диаграммы.

Основные результаты обоснованы в $\S 12$.

Техника из $\S \S 8-12$ применима и в ситуации статьи [8], когда определяющие соотношения строятся без “квадратичной” буквы $x$ на базе других машин. С использованием этой техники и работы [8] будет написана совместная статья с доказательством разрешимости проблемы слов в групе $G$ недетерминистской машиной в полиномиальное время в том и только в том случае, когда группа $G$ вложима в группу с полиномиальной изопериметрической функцией. Свойства построенньх в [8] машин позволят также усилить теорему 3 добавлением, что проблема равенства слов в группе $H$ разрешима тогда и только тогда, когда она разрешима в $R$.

\section{§2. Вложение в рекурсивно определенную группу}

Для вывода теоремы 2 из теорем 1 и 3 нужно объяснить, почему группа $R$ в теореме 1 имеет рекурсивно перечислимое множество определяющих соотношений, если функция $l$ c D-условием на группе $G$ вычислима. C этой целью коротко напомним построение вложения в теореме 1 [1].

Сначала алгоритмически строится некоторое вспомогательное множество положительных слов $\mathscr{M}$ от двух переменных с нужными свойствами типа условия малых сокращений. Это множество экспоненциально, т.е. существует число $c>1$ такое, что в $\mathscr{M}$ не меньше $c^{i}$ слов длины $\leqslant i$ для каждого $i \geqslant i_{0}$, причем числа $c$ и $i_{0}$ можно указать явно.

Далее в лемме 5 [1] строится инъективное соответствие:

$$
g \mapsto X_{g} \in \mathscr{M}
$$

где $g \in G \backslash 1$. Это соответствие определяет вложение группы $G$ в групшу $H$, заданную двумя порождающими и всеми соотношениями вида $X_{g_{1}} X_{g_{2}} \cdots X_{g_{n}}=1$, если $g_{1} g_{2} \ldots g_{n}=1$ в $G$. Повторим здесь доказательство леммы 5 [1], обращая внимание на эффективность нахождения слов $X_{g}$. 
Лемма 2.1. Пусть $\mathscr{M}$ - экспоненциальное множество слов над конечныцм алфавитом $\left\{a_{1}, \ldots, a_{m}\right\}$. Тогда для вычислимой функиии $l: G \rightarrow \mathbb{N} c$ D-условием можсно указать константу $d=d(\mathscr{M}, l)$ и эффективно определить инбективное отображсение (2.1) со свойством:

$$
l(g) \leqslant\left\|X_{g}\right\|<d l(g), \quad g \in G \backslash 1 .
$$

ДокАЗАТЕЛЬСтво. По условию (D3) существует такая константа $a$, что для каждого подмножества $G_{i}=\{g \in G \backslash 1 \mid l(g) \leqslant i\}$

$$
\operatorname{card} G_{i}<a^{i}
$$

Пусть $c$ - константа экспоненциальности множества $\mathscr{M}$. Тогда можно выбрать такое число $d>i_{0}$, что

$$
c^{d i-1}-(2 m+1)^{i} \geqslant a+a^{2}+\cdots+a^{i}, \quad i=1,2, \ldots .
$$

Поскольку число всех слов длины $\leqslant i-1$ меньше $(2 m+1)^{i}$, неравенство $(2.4)$ означает, что множество $\mathscr{M}$ имеет более $a+\cdots+a^{i}$ слов с длинами на отрезке $[i, d i-1]$. Значит, среди этих слов можно выделить, причем эффективно, попарно непересекаюшиеся подмножества $\mathscr{X}_{i 1}, \ldots, \mathscr{X}_{i i}$, состоящие из $a, \ldots, a^{i}$ слов соответственно. (Можно считать, что $a$ - целое.) Сделаем это так, что все те слова из выбранных ранее подмножеств $\mathscr{X}_{i-1,1}, \ldots, \mathscr{X}_{i-1, i-1}$, длины которых принадлежат отрезку $[i, d i-1]$, входили соответственно в $\mathscr{X}_{i 1}, \ldots, \mathscr{X}_{i, i-1}$. (Заметим, что $G_{0}=\varnothing$, как видно из D-условия.) Тогда подмножества $\mathscr{X}_{i i}, i=1,2, \ldots$, попарно не пересекаются.

Заметим, что в силу условия (D1) и вычислимости функции $l$ в группе $G$ алгоритмически разрешима проблема равенства. Значит, ее неединичные элементы (представленные словами в некотором алфавите) можно эффективно пронумеровать. Рассматривая произвольный элемент $g=g_{k}$, вычисляем значение $l(g)=i$ и сопоставляем ему в качестве $X_{g}$ первое, не использованное ранее слово из подмножества $\mathscr{X}_{i i}$. Такое слово всегда найдется в силу условия (D3) и равенства card $\mathscr{X}_{i i}=a^{i}$.

Лемма доказана.

Лемма 2.2. Если данная в теореме 1 функиия $l$ с D-условием вычислима, то группу $R$ можсно задать рекурсивно перечислимым множеством определяющих соотношений.

ДокАЗАТЕЛЬСтво. Как отмечено выше, в группе $G$ алгоритмически разрешима проблема равенства. Поэтому, проверяя последовательно справедливость в ней всех равенств вида $g_{1} \cdots g_{n}=1$ для всевозможных неединичных сомножителей из $G$, получаем на основании леммы 2.1 и определения групшы $R$ перечисление определяющих для $R$ соотношений. 


\section{§3. Гиперболические плоские графы}

Рассмотрим конечньй плоский 2-комплекс, т.е. граф̆ Г на плоскости с вершинами $v_{0}, v_{1}, \ldots, v_{n}$, где $n \geqslant 1$. Выделенная вершина $v_{0}$ считается внешней, а остальные - внутренние. Предполагается, что граф Г так расположен на плоскости, что в нем нет одноугольных граней с вершинами $v_{i}$ и двухугольных граней с вершинами $v_{i}, v_{j}$ при $i, j \geqslant 1$. Нет также петель в $v_{0}$ (но вершина $v_{0}$ вполне может соединяться с другой вершиной несколькими ребрами). Такой граф будем называть $l$-графом, если степень (инцидентности) каждой внутренней вершины не меньше числа $l \geqslant 6$.

Граф̆ Г считается так уложенным на плоскости, что вершина $v_{0}$ действительно является внешней, т.е. из нее можно провести кривую на бесконечность, не пересекая ребер графа Г. Если $p$ - простой замкнутый путь, составленный из ребер графа $\Gamma$, то можно говорить об области $O$, ограниченной этим путем (или внутренней относительно $p$ ). Для каждой вершины $о$ пути $p$ в этом случае определена ее внутренняя степень $d(o, p)$ относительно $p$ - число ребер, выходящих из $о$ внутрь области $О$. Приведем известную “формулу кривизны” (см. следствие 3.3 гл. 5 книги [9] для $(6,3)-$ карт).

ЛЕмма 3.1. Если $o_{1}, \ldots, o_{s}$ - все верииньи, последовательно проходимые простым замкнутыл путем $р$ в l-графе, причем $р$ не проходит через внешнюю вериину, то

$$
\sum_{i=1}^{s}\left(2-d\left(o_{i}, p\right)\right) \geqslant 6
$$

(т.е. в среднем внутренние степени вериин $o_{1}, \ldots, o_{s}$ меньше 2).

Следующее свойство также хорошо известно.

ЛЕмма 3.2. B l-графе Г найдется внутренняя вериина о степени $d \geqslant l u$ не менее $d-3$ последовательных ребер, выходящих из нее (при ее обходе по часовой стрелке), соединяющих о с внешней верииной $v_{0}$ так, что между смежними ребрами нет других вериин графа Г.

ДокАЗАтЕльство. Пусть $o_{1}-$ некоторая внутренняя вершина в Г. Если первое утверждение леммы неверно, ее можно соединить ребром $e_{1}$ с некоторой внутренней вершиной $o_{2}$. Если и для $o_{2}$ первое утверждение неверно, - проводим из $o_{2}$ ребро $e_{2}$ в некоторую внутреннюю вершину оз и т. д. пока не получим простой замкнутый путь $p$.

Сушествует поэтому несократимый замкнутый путь $q$ без самопересечений (с точностью до произвольно малой деформации), который проходит через внутренние вершины и ограничивает максимально возможную область $O$. В силу такой максимальности в нем можно выделить простой замкнутьй подпуть $q^{\prime}$, имеющий не более одной обшей вершины $v$ со своим дополнением $q^{\prime \prime}$ до пути $q$. Но из неравенства (3.1) видно, что на $q^{\prime}$ должна быть по крайней мере еше одна вершина $v^{\prime}$ внутренней степени не больше 1. Если первое утверждение леммы и для нее неверно, то из $v^{\prime}$ можно выпустить в какую-то внутреннюю вершину некоторое ребро $f_{1}$, не лежащее в $O$ или на $q$. Тогда, продолжая построение ребер $f_{2}, \ldots$, можно определить с помощью этих ребер и ребер пути $q$ некоторый путь $q_{0}$ без самопересечений, ограничивающий область большую, чем $O$. 
Итак, в графе Г найдется вершина $o$, удовлетворяюшая первому требованию леммы. Если при этом между смежньми ребрами $e^{\prime}$ и $e^{\prime \prime}$, соединяюшими ее с $v_{0}$, найдутся другие вершины, то задача сводится к подграфу с меньшим числом вершин, который заключен между $e^{\prime}$ и $e^{\prime \prime}\left(\right.$ с $v_{0}$, но без $\left.o\right)$.

Лемма доказана.

ЛЕмма 3.3. $l$-граф связен при любом $l=6,7, \ldots$.

ДокАЗАтЕльство. Иначе некоторая компонента связности не содержит внешнюю вершину, что противоречит предыдушей лемме, ибо $d-3 \geqslant l-3 \geqslant 3>0$.

Лемма 3.4. Пусть $d_{0}, d_{1}, \ldots, d_{n}-$ степени вериин $v_{0}, v_{1}, \ldots, v_{n}$ в $l$-графе $\Gamma$. Тогда $d_{0} \geqslant 6-6 n+\sum_{i=1}^{n} d_{i}$.

ДокАЗАТЕЛЬство. Утверждение верно при $n=1$, так как все вершины, выходящие из $v_{1}$, должны кончаться в $v_{0}$. При $n>1$ можем считать, что именно для вершины $o=v_{n}$ справедливо утверждение леммы 3.2. Рассмотрим граф $\Gamma^{\prime}$ с вершинами $v_{0}, \ldots, v_{n-1}$, в котором ребра графа $\Gamma$ между каждой из вершин $v_{1}, \ldots, v_{n-1}$ и вершиной $v_{n}$ заменены на ребра между $v_{1}, \ldots, v_{n-1}$ и вершиной $v_{0}$. (Это можно сделать, так как вершина $v_{n}$ соединена в $\Gamma$ с $v_{0}$ по лемме 3.2.) Тогда $\Gamma^{\prime}-l$-граф, и по лемме 3.2 кратности $d_{0}$ и $d_{0}^{\prime}$ вершины $v_{0}$ в $\Gamma$ и в $\Gamma^{\prime}$ связаны неравенством $d_{0}^{\prime}-3 \leqslant d_{0}-\left(d_{n}-3\right)$, откуда по предположению индукции

$$
d_{0} \geqslant d_{0}^{\prime}+d_{n}-6 \geqslant \sum_{i=1}^{n-1} d_{i}-6(n-1)+6+d_{n}-6=\sum_{i=1}^{n} d_{i}-6 n+6 .
$$

На стандартной гиперболической плоскости кривая без самопересечений постоянной, но не слишком большой кривизны, вполне может быть неограниченной в обе стороны. Кроме того, две такие кривые, проведенные ортогонально в концах двух отрезков с обшим началом, не пересекаются, если отрезки достаточно длинные, а угол между ними не слишком маленький. Далее в этом параграфе мы рассмотрим некоторые дискретные аналоги этого проявления гиперболичности в конечных графах, на которых базируются оценки в $§ 10$.

Пусть два ребра $e$ и $e^{\prime}$ выходят из некоторой внутренней вершины $v l$-графа $\Gamma$. Тогда в циклическом порядке все ребра, выходящие из $v$, можно записать в виде $e, f_{1}, \ldots, f_{n_{1}}, e^{\prime}, f_{1}^{\prime}, \ldots, f_{n_{2}}^{\prime}$. Мы скажем, что ребра $e$ и $e^{\prime}$ образуют большой угол, если $n_{1} \leqslant 2 n_{2}$ и $n_{2} \leqslant 2 n_{1}$.

Назовем любой путь вида $e_{1} e_{2} \ldots e_{\alpha}$ в $Г$ мало искривленныцм, если концы ребер $\left(e_{1}\right)_{+}, \ldots,\left(e_{\alpha-1}\right)_{+}-$внутренние вершины, а ребра $\left(e_{i}\right)^{-1}$ и $e_{i+1}$ составляют большой угол в вершине $\left(e_{i}\right)_{+}=\left(e_{i+1}\right)_{-}$для всех $i=1, \ldots, \alpha-1$.

ЛЕмма 3.5. Если мало искривленный путь р начинается во внутренней вериине $\left(e_{1}\right)_{-}$, то он не может быть замкнутым в $l$-графе при любом $l \geqslant 6$.

ДокАЗАТЕльство. Выбирая при доказательстве "от противного" длину $\alpha$ пути $p$ минимальной, можем считать, что $p$ - простой путь. Ввиду его малой искривленности каждая вершина цикла $p$, кроме может быть одной - вершины $p_{-}=p_{+}$, имеет на $p$ внутреннюю степень не меньше 2 . Но это противоречит неравенствy (3.1). 
Из леммы 3.5 следует, что максимально продолженный в обе стороны мало искривленный путь в $l$-графе должен начинаться и кончаться во внешней вершине $v_{0}$.

Пусть $x$ - некоторая жорданова дуга на плоскости, имеющая с графом $\Gamma$ ровно две обшие точки, являюшиеся вершинами графа: $x_{-}$и $x_{+}$. Назовем ее $\Gamma-n p a-$ вильной. Пусть простой путь $p$ в Г проходит через вершину $v$, равную $x_{-}$или $x_{+}$. Тогда все ребра, выходящие из $v$, можно циклически выписать в виде $f_{1}, \ldots, f_{d}$, так что дуга $x$ входит в $v$ между $f_{d}$ и $f_{1}$. Скажем, что она $k$-отделен $a$ от $p$, если ребра $f_{1}, \ldots, f_{k}$ и $f_{d-k+1}, \ldots, f_{d}$ (и им обратные) не встречаются в пути $p$.

Два максимальных мало искривленных пути $p$ и $p^{\prime}$ назовем расходящимися в вершинах $v$ и $v^{\prime} l$-графа $\Gamma$, если некоторые две различные их внутренние вершины $v$ и $v^{\prime}$ соответственно можно соединить Г-правильной дугой $x$, которая 2-отделена от $p$ и от $p^{\prime}$.

ЛЕмма 3.6. Два расходящихся пути не имеют общих внутренних в l-графе Г вершин.

ДокАЗАТЕЛЬСТво. Во введенных выше обозначениях пусть граф $Г(x)$ совпадает с $\Gamma$, если дуга $x$ образует двухугольник вместе с некоторым ребром $e$ в $\Gamma$ и получается из $\Gamma$ добавлением дуги $x$ в качестве нового ребра в противном случае. Очевидно, что $\Gamma(x)$ - тоже $l$-граф.

Если $p$ и $p^{\prime}$ имеют общую внутреннюю вершину, то из подпутей путей $p, p^{\prime}$ и пути $x$ (или ребра $e$ ) можно составить простой замкнутый путь $q$ в $\Gamma(x)$, не проходящий через внешнюю вершину. В силу малой искривленности путей $p, p^{\prime}$ не более трех вершин пути $q$ могут дать положительный вклад в левую часть неравенства (3.1): $x_{-}=v, x_{+}=v^{\prime}$ и обшая вершина путей $p, p^{\prime}$. Но внутренняя степень каждой из вершин $x_{-}, x_{+}$на $q$ не меншше 1 (даже при замене $x$ на $e$ в $\Gamma(x)$ ввиду условия 2 -отделенности в определении расходящихся путей). Поэтому левая часть в (3.1) меньше 6 вопреки утверждению леммы 3.1 .

Полученное противоречие доказьвает лемму.

По лемме 3.5 максимальный мало искривленный путь $p$ в $l$-графе $\Gamma$ является простым, а значит, разделяет две области $O_{1}$ и $O_{2}$ на плоскости (внутреннюю и внешнюю). Если из каждой вершины $v \neq v_{0}$ на $p$ в область $O_{1}$ (в область $O_{2}$ ) направлено по крайней мере на восемь ребер больше, чем в $O_{2}$ (чем в $O_{1}$ ), то путь $p$ назовем овалом и обозначим $O(p)=O_{1}\left(O(p)=O_{2}\right)$. (Легко понять, что овалы могут встречаться только при $l=26$ или $l \geqslant 28$.)

Назовем некоторую простую плоскую жорданову кривую Г-трансверсальной, если она не проходит через вершины графа Г и трансверсально пересекает ребра этого графа. Скажем, что Г-трансверсальная кривая огибает некоторую внутреннюю вершину графа $\Gamma$, если она последовательно пересекает выходящие из $v$ peбра $f_{1}, \ldots, f_{s}$, где $s-2$ не меньше половины степени вершины $v$, причем в образовавшихся между смежными ребрами и этой кривой “секторах" нет других вершин графа Г.

ЛЕмма 3.7. Пусть Г-трансверсальная кривая х челиком лежит в области $O(p)$, определенной овалом $p$ l-графа $\Gamma$, кроме точек $x_{-} u x_{+}$, лежащих на р. Тогда или (1) $x$ пересекает подряд дважды одно и то же ребро $f$ из $Г$, так что в ограниченной $x$ и $f$ области нет вершин графа $Г$, или (2) $x$ огибает некоторую внутреннюю вериину графа Г. 
ДокАЗАТЕЛЬСтво. Допустим, что ситуация (1) не реализуется и обозначим $\bar{O}$ замкнутую область на плоскости, ограниченную кривой $x$ и овалом $p$, не содержащую внешней вершины $v_{0}$.

Предположим сначала, что найдется некоторая последовательность ребер $f_{1}, \ldots, f_{s}$ в $\Gamma$, последовательно пересекаемых кривой $x$, где $f_{1}$ и $f_{s}$ выходят из одной вершины $о$ графа $\Gamma$, лежашей в $\bar{O}$, но не все векторы этой последовательности инцидентны вершине $o$. (Назовем здесь $o$ ᄎ-вершиной.) Пусть вершина $o$ выбрана так, что число вершин в области, ограниченной с помощью $f_{1}, f_{s}$ и $x$, минимально возможное.

Рассмотрим вспомогательный граф $\Gamma(o)$, вершинами которого служат $o, v_{0}$ и все вершины внутри области $U$, ограниченной ребрами $f_{1}, f_{s}$ и заключенной между ними дугой кривой $x$ (рис. 1a). Такие вершины тоже есть по выбору вершины $o$. Все выходящие из $о$ ребра в $\Gamma$, не направленные внутрь $U$, перенаправим в графе $\Gamma(o)$ в вершину $v_{0}$.

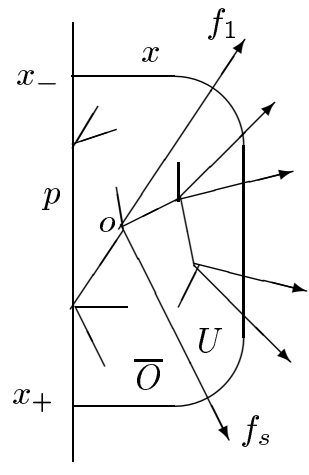

a

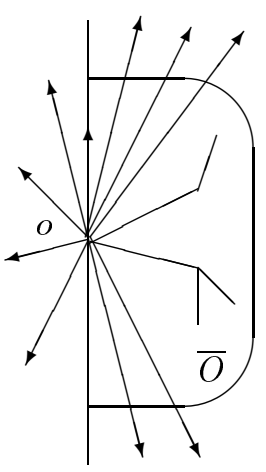

б

Рис. 1

Заметим, что сектор, ограниченный частью дуги кривой $x$, заключенной между смежньми ребрами какой-либо вершины внутри $U$, не может заключать других вершин из $\Gamma$, так как иначе возник бы в этом секторе $l$-граф, у которого не более одного внешнего ребра по выбору вершины $o$, но вопреки лемме 3.2. Поэтому, если $x$ не огибает никакой вершины в $l$-графе $\Gamma(o)$, то число внешних ребер каждой вершины, кроме $v_{0}$, в нем не превосходит половины ее степени плюс единицы по выбору вершины $o$. Но это противоречит лемме 3.4 для $\Gamma(o)$, так как $l \geqslant 26$, как было замечено при определении овалов.

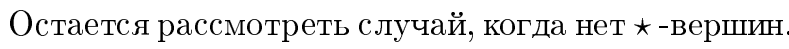

В новый граф $Г(O)$ включим внешнюю вершину $v_{0}$ и все вершины, лежашие в $\bar{O}$. Оставим все старые ребра, находящиеся в $\bar{O}$, а каждое ребро, выходящее из $\bar{O}$ во вне, заменим на ребро с концом в $v_{0}$. Очевидно, что $\Gamma(O)$ - тоже $l$-граф, так как в $\bar{O}$ есть хотя бы одна вершина.

По лемме 3.2 в $\Gamma(O)$ найдется вершина $o$ степени $d \geqslant l$, у которой не менее $d-3$ последовательных ребер являются внешними (и между ними нет вершин графа $\Gamma(O))$. Из них не более $\frac{1}{2}(d-2)-4$ могут выходить из $o$, не пересекая пути $x$, как видно из определения овала. Значит, оставшиеся, число которых не меньше 
$\frac{1}{2} d+2$, пересекаются кривой $x$, причем последовательно (а не как на рис. 1б), ибо $o$ не является $\star$-вершиной. Итак, вершина $о$ огибается кривой $x$.

Рассмотрим теперь произвольный овал $p$ в $l$-графе $\Gamma$, проходяший через некоторую внутреннюю вершину $v$. Пусть $v^{\prime} \neq v$ - другая внутренняя вершина, расположенная в замыкании области $O(p)$, определенной этим овалом, a $p^{\prime}$ - овал, проходящий через $v^{\prime}$. Если овалы $p$ и $p^{\prime}$ являются расходящимися в вершинах $v$ и $v^{\prime}$, причем соответствующая Г-правильная дуга $x$-отделена от $p^{\prime}$ и не лежит в замыкании области $O\left(p^{\prime}\right)$, то овал $p^{\prime}$ назовем производным от овала $p$ в вершинах $v, v^{\prime}$. По лемме 3.6 вершина $v^{\prime}$ не может при этом лежать на $p$.

Лемма 3.8. (1) В приведенных обозначениях область $O(p)$ содержит область $O\left(p^{\prime}\right)$, определенную производным овалом $p^{\prime}$.

(2) Пусть $p^{\prime \prime}$ - другой производньй овал от овала $р$ в вершинах $v$ и $v^{\prime \prime}$, причем $v^{\prime \prime} \neq v^{\prime}$. Тогда области $O\left(p^{\prime \prime}\right)$ и $O\left(p^{\prime}\right)$ не пересекаются.

ДокАЗАТЕЛЬСтво. (1) Первое утверждение следует из леммы 3.6 и того, что $x$ не лежит в $\overline{O\left(p^{\prime}\right)}$.

(2) Из леммы 3.6 и данной по определению возможности соединить вершины $v$ и $v^{\prime}$ Г-правильной дугой следует, что область $O\left(p^{\prime}\right)$ не может содержаться в $O\left(p^{\prime \prime}\right)$. Верно и обратное; поэтому, доказывая лемму “от противного", можем считать, что овалы $p^{\prime}$ и $p^{\prime \prime}$ имеют общую внутреннюю вершину о. (См. рис. 2, но возможности $o=v^{\prime}$ или $o=v^{\prime \prime}$ пока не исключаются.)

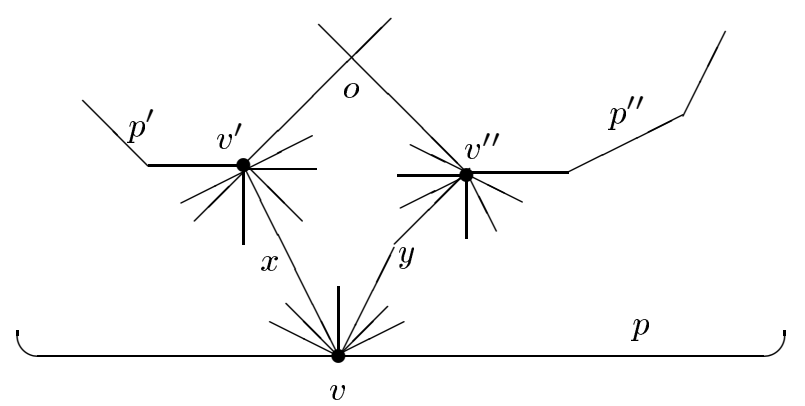

Рис. 2

Введем вспомогательньй граф $\Gamma(x, y)$, добавляя к графу Г дуги $x$ и $y$ или не добавляя их, как в доказательстве леммы 3.6, где $x, y-\Gamma$-правильные дуги между $v, v^{\prime}$ и $v, v^{\prime \prime}$ соответственно. Как и в лемме 3.6 , составим простой замкнутый путь $q$, проходящий только через внутренние вершины графа $\Gamma$, из $x$ (или ребра $e$, если $x$ не включили в $\Gamma(x, y)), y$ (или $e^{\prime}$ ) и подпутей овалов $p$ и $p^{\prime}$ от вершин $v$ и $v^{\prime}$ до вершины $o$. По определению мало искривленных путей $p$ и $p^{\prime}$ и по условию 3-отделенности дуг $x, y$ от $p^{\prime}, p^{\prime \prime}$ соответственно только две вершины на $q$, а именно $v$ и $o$, могут иметь внутренние степени вдоль $q$ меньшие, чем 2. Но это противоречит лемме 3.1, примененной к $q$.

Следовательно, второе утверждение леммы также верно. 


\section{$\S 4$. Список соотношений}

Пусть группа из теоремы 3 имеет копредставление

$$
R=\left\langle a_{1}, \ldots, a_{m} \mid w=1, w \in E\right\rangle
$$

где $E$ - рекурсивно перечислимое множество слов от $a_{1}, \ldots, a_{m}$. Добавляя, если нужно, новые порождающие и соотношения вида $a_{j} a_{j}^{\prime}=1$, можно считать в дальнейшем, что множество $E$ состоит только из положсительных слов, т.е. слова $w \in E$ не содержат вхождений вида $a_{j}^{-1}$.

Построим серию групп по копредставлению (4.1), следуя изложению из книги Ротмана [6] и изменяя лишь одно соотношение (4.10).

Напомним коротко [6, гл. 12], что ввиду положительности слов из $E$ и рекурсивной перечислимости этого множества имеется некоторая детерминистская машина Тьюринга $T$ с одной головкой, одной лентой и ленточньм алфавитом $\left\{s_{0}, s_{1}, \ldots, s_{M}\right\}$, содержащим $a_{1}, \ldots, a_{m}$, которая останавливается (при этом очищая ленту и переходя в заключительное состояние $q$ ) тогда и только тогда, когда поданное на вход в начале ее работы положительное слово $w$ от $s_{0}, \ldots, s_{M}$ принадлежит множеству $E$.

По машине $T$ строится полугруппа Маркова - Поста $\Gamma(T)$ с порождающими $s_{0}, \ldots, s_{M}, h, q, q_{0}, \ldots, q_{N}$, где буква $h$ отмечает край ленты, а буквы $q, q_{0}, \ldots, q_{N}$ отвечают возможным состояниям машины $T$. Она обладает следующим свойством [6, лемма 12.4].

Лемма 4.1. Равенство $h q_{1} w h=q$ для слова $w$ в алфавите $s_{0}, \ldots, s_{M}$ справедливо в полугруппе $\Gamma(T)$ тогда и только тогда, когда $w \in E$.

Обозначим через $B_{0}=\langle x\rangle$ бесконечную циклическую группу с порождаюшим $x$. Группа $B_{1}$ задается порождающими $x, h, s_{0}, \ldots, s_{M}$ и соотношениями

$$
h^{-1} x h=x^{2}, \quad s_{\beta}^{-1} x s_{\beta}=x^{2}, \quad \beta=0, \ldots, M .
$$

Определение группы $B_{2}$ и последуюших зависит уже от команд машины $T$. Для задания групшы $B_{2}$ к порождаюшим групшы $B_{1}$ добавляются буквы $r_{i}$ (отвечающие различным командам машины $T$ ), где $i \in I, I$ - конечное множество, а к соотношениям (4.2) добавляются соотношения

$$
\begin{gathered}
r_{i}^{-1} s_{\beta} x r_{i}=s_{\beta} x^{-1}, \quad i \in I, \quad \beta=0, \ldots, M \\
r_{i}^{-1} h x r_{i}=h x^{-1}, \quad i \in I, \\
r_{i}^{-1} F_{i}^{\#} q_{i_{1}} G_{i} r_{i}=H_{i}^{\#} q_{i_{2}} K_{i}, \quad i \in I .
\end{gathered}
$$

Здесь соотношения (4.5) выписаны по одному для каждого $i \in I$, в них $q_{i_{1}}, q_{i_{2}} \in$ $\left\{q, q_{0}, \ldots, q_{N}\right\}\left(q_{i_{1}}\right.$ - состояние перед выполнением $i$-й команды, а $q_{i_{2}}$ - после ее вьполнения); $F_{i}, G_{i}, H_{i}, K_{i}$ - положительные (возможно, пустые) $(s, h)$-слова (т.е. слова в алфавите $\left\{s_{0}, \ldots, s_{M}, h\right\}$ ), “ " $V$ \#" означает переписывание слова $V$ с заменой каждой его буквы на обратную. При этом суммарное число вхождений $s$-букв в эти четыре слова не превышает четырех. Их перечень, определяемый командами машины $T$, читатель при желании может найти в [6]. В частности, среди них есть 
соотношение вида $r_{i_{0}}^{-1} h^{-1} q_{0} h r_{i_{0}}=q$ и нет других соотношений с буквой $q$ или с подсловом $h^{-1} q_{0} h$. Буква $h$ входит не более одного раза в каждое из слов $F_{i}, G_{i}$, $H_{i}, K_{i}$. Она может быть только первой у слов $F_{i}, H_{i}$ и только последней у слов $G_{i}, K_{i}$, причем слово $F_{i}$ (слово $G_{i}$ ) начинается с буквы $h$ (кончается на $h$ ) тогда и только тогда, когда этим же свойством обладает слово $H_{i}\left(\right.$ слово $\left.K_{i}\right)$.

В [6] можно найти и следующие несложные факты (лемма 12.11, (i)-(iii)).

Лемма 4.2. В иепи $B_{0} \leqslant B_{1} \leqslant B_{1} * Q \leqslant B_{2}$, әде $Q-$ свободная группа с базисом $\left\{q_{0}, \ldots, q_{N}\right\}$, каждая группа является $H N N$-расширением предьдущей. Точнее

(1) $B_{1}$ есть HNN-расширение с базой $B_{0}$ и проходными (stable) буквами $h, s_{0}, \ldots, s_{M}$

(2) $B_{1}$ и $Q$ действительно порождают свое свободное произведение в $B_{2}$;

(3) $B_{2}$ есть $H N N$-расширение с базой $B_{1} * Q$ и проходными буквами $r_{i}, i \in I$.

ЛЕмма 4.3. Пусть для некоторого $i \in I$ H означает подгруппу группь $B_{2}$, порождденную либо әлементами $F_{i}^{\#} q_{i_{1}} G_{i}, h x$ и всеми $s_{\beta} x, \beta=0, \ldots, M$, либо әлементами $H_{i}^{\#} q_{i_{2}} K_{i}, h x^{-1}$ и $s_{\beta} x^{-1}, \beta=0, \ldots, M, a K=g p\left\{x, r_{i} ; i \in I\right\}$. Тогда $H \cap K=1$, а подгруппь $H$ и $K$ свободно порождаются указанными әлементами.

ДоказАтеЛЬство. Рассмотрим в $B_{2}$ равенство вида $V=U$, где $V$ - приведенное слово от $x$ и $r_{i}, i \in I$, а $U$ - произведение порождаюших группы $H$ и им обратных. Если $V$ содержит некоторую букву $r_{i}$, то поскольку $U$ не содержит таких букв, по лемме 4.2 и лемме Бриттона в слове $V$ есть подслово вида $r_{i}^{ \pm 1} x^{n} r_{i}^{\mp 1}$, где $x^{n} \in H$ и $n \neq 0$. Но ввиду свойств (1), (2) леммы 4.2 сушествует эндоморфизм групшы $B_{1} *\left\langle q_{i_{1}}\right\rangle$ (вариант с $q_{i_{2}}$ вполне аналогичен), при котором $x \mapsto 1, q_{i_{1}} \mapsto q_{i_{1}}$, $s_{\beta} \mapsto s_{\beta}, h \mapsto h$. Под действием этого гомоморфизма элемент $x^{n}$ перейдет в 1 , а его запись через порождаюшие группы $H$, представляюшая нетривиальное слово $\left(\right.$ ибо $\left.x^{n} \neq 1\right)$, перейдет в нетривиальный элемент, так как $q_{i_{1}}, s_{\beta}(\beta=0, \ldots, M)$ и $h$ свободно порождают свободную подгруппу в силу леммы 4.2 .

То же самое рассуждение показьвает, что $V=1$, если слово $V$ не зависит от букв $r_{i}, i \in I$, и что $H, K$ - свободные подгрупшы.

Приведем еще утверждения лемм 12.14 и 12.15 [6].

Лемма 4.4. Пусть $L_{1}$ и $L_{2}$ - несократимье слова в алфавите $\left\{x, r_{i} ; i \in I\right\}$, a $X, Y$ - несократимые $(s, h)$-слова. Тогда если $L_{1} X^{\#} q_{j} Y L_{2}=q$ в группе $B_{2}$, где $q_{j} \in\left\{q, q_{0}, \ldots, q_{N}\right\}$, то оба слова $X, Y$ положительны и $X q_{j} Y=q$ в полуzpynne $\Gamma(T)$.

Зафиксируем теперь целое число $L \geqslant 15$. (В [6] $L=1$.) Копредставление группы $B_{3}$ получается, если к порождающим группы $B_{2}$ добавить буквы $t_{1}, \ldots, t_{L}$, а к соотношениям добавить следующие:

$$
\begin{gathered}
t_{j}^{-1} r_{i} t_{j}=r_{i}, \quad i \in I, \quad j=1, \ldots, L, \\
t_{j}^{-1} x t_{j}=x, \quad j=1, \ldots, L .
\end{gathered}
$$


В свою очередь, из этого копредставления получается копредставление группы $B=B(T)$, если к копредставлению группы $B_{3}$ добавить порождающие $k_{1}, \ldots, k_{L}$ и соотношения

$$
\begin{gathered}
k_{j}^{-1} r_{i} k_{j}=r_{i}, \quad i \in I, \quad j=1, \ldots, L, \\
k_{j}^{-1} x k_{j}=x, \quad j=1, \ldots, L \\
\prod_{j=1}^{L} k_{j}^{-1}\left(q^{-1} t_{j} q\right) k_{j}\left(q^{-1} t_{j}^{-1} q\right)=1 .
\end{gathered}
$$

Под $h$-специальньм словом понимается слово $\Sigma \equiv X_{0}^{\#} q_{j} Y_{0}$, где $X_{0} \equiv h X$, $Y_{0} \equiv Y h, X, Y$-положительные слова в алфавите $\left\{s_{0}, \ldots, s_{M}\right\}, q_{j} \in\left\{q_{0}, \ldots, q_{N}\right\}$, a "三” - знак побуквенного равенства. В этом случае по определению $\Sigma^{*} \equiv X_{0} q_{j} Y_{0}$. Сделанная выше модификация соотношения (4.10) не меняет доказательства достаточности в лемме Буна (см. [6]).

Лемма 4.5. Если $\Sigma-h$-специальное слово и $\Sigma^{*}=q$ в полугруппе $\Gamma(T)$, то

$$
\prod_{j=1}^{L} k_{j}^{-1}\left(\Sigma^{-1} t_{j} \Sigma\right) k_{j}\left(\Sigma^{-1} t_{j}^{-1} \Sigma\right)=1
$$

в zpynne $B$.

Изменение в соотношении (4.10) влечет ниже изменения в определении групп Андера $B_{4}, B_{5}, B_{6}$ по сравнению с [6].

Запишем копредставление (4.1) группы $R$ в новых порождающих:

$$
R \cong R_{u}=\left\langle u_{1}, \ldots, u_{m} \mid w_{u}=1, w_{u} \in E_{u}\right\rangle
$$

где нижний индекс “ $u$ ” означает переписывание слова $w=w\left(a_{1}, \ldots, a_{m}\right)$ как $w\left(u_{1}, \ldots, u_{m}\right)$ после подстановок $a_{j} \mapsto u_{j}$.

Пусть $B * R_{u}$ - свободное произведение. К его естественному копредставлению с определяюшими соотношениями (4.2)-(4.11) добавим порождаюшие $b_{1}, \ldots, b_{m}$ и соотношения (4.12)-(4.14), задаюшие группу $B_{4}$ (напомним, что каждая буква $a_{j}$ входит в $\left.\left\{s_{0}, \ldots, s_{M}\right\}\right)$ :

$$
\begin{aligned}
b_{i}^{-1} u_{j} b_{i} & =u_{j}, \quad i, j=1, \ldots, m ; \\
b_{i}^{-1} a_{j} b_{i} & =a_{j}, \quad i, j=1, \ldots, m ; \\
b_{i}^{-1} h k_{1} h^{-1} b_{i} & =h k_{1} h^{-1} u_{i}, \quad i=1, \ldots, m .
\end{aligned}
$$

ЛЕмМа 4.6. Подгруппьи $g p\left\{u_{1}, \ldots, u_{m}\right\}$ u $g p\left\{b_{1}, \ldots, b_{m}\right\}$ nорожсдают в группе $\mathrm{B}_{4}$ свое прямое произведение. 
ДокАЗАТЕЛЬСТво. Гомоморфизм группы $B_{4}$ на свободную группу с базисом $\left(x_{1}, \ldots, x_{m}\right)$, при котором $b_{i} \mapsto x_{i}$, а порождающие группы $B * R_{u}$ отображаются в 1 , показывает тривиальность пересечения указанных подгрупп. Поэтому утверждение следует из соотношений (4.12).

Добавляя к полученному копредставлению группы $B_{4}$ новый порождающий $d$ и соотношения (4.15), (4.16), получим группу $B_{5}$ :

$$
\begin{gathered}
d^{-1} h k_{1} h^{-1} d=h k_{1} h^{-1}, \\
d^{-1} a_{i} b_{i} d=a_{i}, \quad i=1, \ldots, m .
\end{gathered}
$$

Наконец, группа $B_{6}$ получается после добавления к $B_{5}$ порождаюшего $\sigma$ и соотношений (4.17)-(4.20):

$$
\begin{gathered}
\sigma^{-1}\left(q_{1}^{-1} h t_{1} h^{-1} q_{1}\right) \sigma=\left(q_{1}^{-1} h t_{1} h^{-1} q_{1}\right) d, \\
\sigma^{-1}\left(q_{1}^{-1} h t_{j} h^{-1} q_{1}\right) \sigma=q_{1}^{-1} h t_{j} h^{-1} q_{1}, \quad j=2, \ldots, L, \\
\sigma^{-1}\left(h k_{j} h^{-1}\right) \sigma=h k_{j} h^{-1}, \quad j=1, \ldots, L \\
\sigma^{-1} a_{i} \sigma=a_{i}, \quad i=1, \ldots, m .
\end{gathered}
$$

Для доказательства теоремы 3 мы установим, что естественный гомоморфизм $R_{u} \rightarrow B_{6}$ является инъективньм (лемма 10.1) и квазиизометричным (§12). Пока же приведем аналог леммы 12.26 [6].

Лемма 4.7. Группа $B_{6}$ может быть задана конечным множеством определяющих соотношений.

ДокАЗАТЕЛЬСТво. Достаточно показать, что каждое соотношение $w_{u}=1$ из списка (4.11) следует из соотношений (4.2)-(4.10), (4.12)-(4.20). Ниже $w_{b}$ - слово, полученное из слова $w=w\left(a_{1}, \ldots, a_{m}\right)$ подстановкой $a_{i} \mapsto b_{i}$ для $i=1, \ldots, m$.

По лемме 4.1 для слова $w=w\left(a_{1}, \ldots, a_{m}\right) \in E$ в полугруппе $\Gamma(T)$ имеем равенство $h q_{1} w h=q$, а значит, по лемме 4.5 , примененной к $\Sigma \equiv h^{-1} q_{1} w h$,

$$
h k_{1}^{-1} h^{-1} w^{-1} q_{1}^{-1} h t_{1} h^{-1} q_{1} w h k_{1} h^{-1}=h P^{-1} h^{-1} w^{-1} q_{1}^{-1} h t_{1} h^{-1} q_{1} w
$$

в группе $B$, где

$$
P \equiv \prod_{j=2}^{L} k_{j}^{-1}\left(h^{-1} w^{-1} q_{1}^{-1} h\right) t_{j}\left(h^{-1} q_{1} w h\right) k_{j}\left(h^{-1} w^{-1} q_{1}^{-1} h\right) t_{j}^{-1}\left(h^{-1} q_{1} w h\right) .
$$

Умножим обе части равенства (4.21) слева на $\sigma^{-1}$, а справа на $\sigma$. В силу соотношений (4.18), (4.19) и (4.20) буква $\sigma$ перестановочна с $h k_{1}^{-1} h^{-1}$, с $h P^{-1} h^{-1}$ и с $w$. Поэтому

$h k_{1}^{-1} h^{-1} w^{-1} \sigma^{-1}\left(q_{1}^{-1} h t_{1} h^{-1} q_{1}\right) \sigma w h k_{1} h^{-1}=h P^{-1} h^{-1} w^{-1} \sigma^{-1}\left(q_{1}^{-1} h t_{1} h^{-1} q_{1}\right) \sigma w$, что в силу (4.17) дает

$$
h k_{1}^{-1} h^{-1} w^{-1}\left(q_{1}^{-1} h t_{1} h^{-1} q_{1}\right) d w h k_{1} h^{-1}=h P^{-1} h^{-1} w^{-1}\left(q_{1}^{-1} h t_{1} h^{-1} q_{1}\right) d w .
$$


Замена $P^{-1}$ по лемме 4.5 на

$$
k_{1}^{-1}\left(h^{-1} w^{-1} q_{1}^{-1} h\right) t_{1}\left(h^{-1} q_{1} w h\right) k_{1}\left(h^{-1} w^{-1} q_{1}^{-1} h\right) t_{1}^{-1}\left(h^{-1} q_{1} w h\right)
$$

приводит после сокращений к равенству $d w h k_{1} h^{-1}=w h k_{1} h^{-1} w^{-1} d w$ или

$$
d\left(w h k_{1} h^{-1} w^{-1}\right) d^{-1}=w h k_{1} h^{-1} w^{-1} .
$$

Левая часть этого соотношения с помошью (4.16), (4.13) и (4.15) может быть переписана в виде $w w_{b} h k_{1} h^{-1} w_{b}^{-1} w^{-1}$. Отсюда $w_{b} h k_{1} h^{-1} w_{b}^{-1}=h k_{1} h^{-1}$, т.е. $\left(h k_{1}^{-1} h^{-1}\right) w_{b}^{-1}\left(h k_{1} h^{-1}\right)=w_{b}^{-1}$. В то же время

$$
\left(h k_{1}^{-1} h^{-1}\right) w_{b}^{-1}\left(h k_{1} h^{-1}\right)=w_{u}^{-1} w_{b}^{-1}
$$

в силу (4.14) и (4.12). Отсюда $w_{u}=1$, что и требовалось.

\section{§5. Диаграммы, полосы, кольца}

Напомним известную топологическую интерпретацию Ван Кампена-Линдона вывода следствий из определяюших соотношений в группах.

Под диаграммой Ван Кампена над некоторым копредставлением группы $G=$ $\left\langle x_{1}, \ldots, x_{k} \mid R_{1}, R_{2}, \ldots\right\rangle$ с циклически несократимыми определяющими словами $R_{1}, R_{2}, \ldots$ (или короче, хотя и менее точно, - над группой $G$ ) понимается конечный плоский ориентированный связный и односвязный 2-комплекс, каждому ориентированному ребру е которого сопоставлена метка $\varphi(e) \equiv x_{i}^{ \pm 1}, i=1, \ldots, k$, причем $\varphi\left(e^{-1}\right) \equiv \varphi(e)^{-1}$, а слово, читаемое на контуре $\partial П$ произвольной 2 -клетки П (просто клетки в дальнейшем), совпадает с точностью до циклической перестановки и взятия обратного с одним из определяюших слов $R_{1}, R_{2}, \ldots$.

Согласно лемме Ван Кампена слово $w=w\left(x_{1}, \ldots, x_{k}\right)$ равно 1 в группе $G$ тогда и только тогда, когда сушествует диаграмма (Ван Кампена) $\Delta$ над $G$, метка граничного контура $\partial \Delta$ которой побуквенно равна $w$, если читать ее, обходя $\partial \Delta$ по часовой стрелке, начиная с некоторой вершины. За подробностями отсылаем читателя к [9] или к [10].

Если в граничную метку некоторой клетки входит какая-то буква $x^{ \pm 1}$ или буква из некоторого подмножества букв $X^{ \pm 1}$, то для краткости такую клетку будем называть $x$-клеткой (соответственно $X$-клеткой). Например, к $t_{j}$-клеткам (или короче, $t$-клеткам, если значение $j$ не существенно) относится любая клетка, отвечаюшая соотношениям $(4.6),(4.7),(4.10),(4.17)$ или $(4.18)$, a $(t, r)$-клетки отвечают только соотношениям (4.6). Если в соотношение входит одна из букв $q, q_{0}, \ldots, q_{N}$, то соответствующие клетки будем называть $Q$-клетками, а клетки, отвечающие соотношениям (4.11), назовем $(u, u)$-клетками, чтобы отличить их от $(u, b)$-клеток, отвечающих соотношениям (4.12) или (4.14).

По лемме 4.7 группа $B_{6}$ конечно определяема, а соотношения (4.10) можно было бы исключить из списка определяющих. Однако оказывается целесообразньм этого не делать, так как в $\S 9$ важной является возможность замены некоторых пар $(k, t)$-клеток другими клетками, в частности, $(u, u)$-клетками. Аналогично, при наличии некоторых конфигураций в диаграммах удается понизить число 
$\sigma$ - или $r$-клеток. Поэтому удобно подсчитывать отдельно число клеток каждого типа в диаграммах (которые таким образом становятся градуированными в смысле $\S 13[10])$.

Самый высокий ранг будут иметь $(k, t)$-клетки, к которым относятся и так называемые диски, определяемые в $\S 7$. Это означает, что из двух диаграмм больший mun имеет по определению та, в которой большее число $(k, t)$-клеток.

Следующими по старшинству считаются $(\sigma, t)$-клетки, т.е. при одинаковом (например, нулевом) числе $(k, t)$-клеток в двух диаграммах больший тип имеет та, в которой больше $(\sigma, t)$-клеток. Далее по старшинству идут $(r, Q)$-клетки. После этого подсчитывается общее число всех остальных клеток. При сравнении типов двух диаграмм их число учитывается в последнюю очередь.

Очевидно, что по типу диаграммы можно вести индуктивные рассуждения. (Строго говоря, тип можно определить как конечный кортеж $\tau(\Delta)=\left(\tau_{1}, \ldots\right)$, где $\tau_{1}$ - число $(t, k)$-клеток в диаграмме $\Delta$ и т. д.)

Диаграмма $\Delta$ называется минимальной, если она имеет наименьший тип среди всех диаграмм с фиксированной граничной меткой.

ЛЕмма 5.1. Пусть в диаграмме $\Delta$ есть две клетки П и П', на контурах ӘП и ӘП' которых при обходе их в противоположных направлениях, начиная с вериин о и о', читается одно и то же слово $V$. Пусть в $\Delta$ имеется путь $p=о-o^{\prime}$ без самопересечений, меткой которого является слово $U$, коммутирующее $c V$ в силу соотношений меньиих рангов, чем ранг $r$ клеток П, П'. Тогда диаграмма $\Delta$ не является минимальной, т.е. число клеток ранга $r$ мохсно уменьшить на 2 при сохранении граничной метки (и чисел клеток больиих рангов).
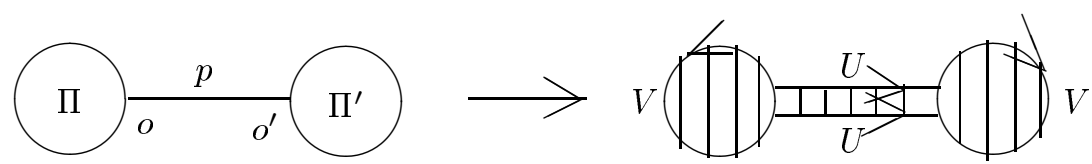

Рис. 3

ДоКАЗАТЕЛЬСТво. Понизить тип диаграммы можно с помощью разреза вдоль пути $p$, удаления клеток П, П' и вклеивания в образовавшуюся дыру диаграммы, состоящей из клеток меньших рангов, интерпретируюшей вывод соотношения $U V U^{-1} V^{-1}=1$ (рис. 3). Более аккуратное объяснение имеется в [10, $\left.\S 13\right]$. (В частности, почему край дыры можно сделать гомеоморфным окружности с помощью предварительного “0-измельчения” диаграммы $\Delta$. .)

Некоторые условия и свойства диаграмм удобно формулировать, используя двойственные понятия. Для этой цели внутри каждой клетки П фиксируется точка $o_{\Pi}$, а вне диаграммы $\Delta$ фиксируется на плоскости некоторая точка $o_{\Delta}$. Аналогично фиксируются точки $o_{e}$ внутри каждого ребра $e$ диаграммы $\Delta$. Если $e-$ ребро на $\partial \Pi$ (на $\partial \Delta)$, то фиксируется простая жорданова дуга $l(\Pi, e)(l(\Delta, e)$ соответственно), соединяющая вершину $o_{\Pi}\left(o_{\Delta}\right)$ с $o_{e}$, не имеющая других общих точек с ребрами диаграммы $\Delta$, кроме $o_{e}$. Требуется также, чтобы $l(\Pi, e)$ и $l\left(\Pi, e^{\prime}\right)$ имели обшей лиш точку оп при $e \neq e^{\prime}$. Аналогичное требование относится к лини$\operatorname{sм} l(\Delta, e)$. 
Для фиксированного множества букв $Y$ (или одной буквы) и некоторого множества определяющих слов $\mathscr{R}$ такого, что каждое слово из $\mathscr{R}$ содержит ровно одно вхождение буквы из $Y$ с показателем 1 и одно - с показателем -1 , определяется понятие $Y$-полосы $S=\left[\pi_{1}, \ldots, \pi_{n}\right]$ как поддиаграммы, составленной из клеток $\pi_{1}, \ldots, \pi_{n}$. Именно, требуется, чтобы (1) каждая клетка $\pi_{1}, \ldots, \pi_{n}$ отвечала одному из соотношений множества $\mathscr{R} ;(2)$ клетки $\pi_{i}$ и $\pi_{i+1}$ были склеены в $S$ по обшему ребру $e_{i}$, где $e_{i} \neq e_{i+1}$ для $i=1, \ldots n-1$, и это ребро является $Y$-ребром, т.е. $\varphi\left(e_{i}\right) \in Y^{ \pm 1}$

Таким образом, граничный контур $\partial S$ полосы $S$ разбивается по определению в произведение вида $\left(e_{0}\right)^{-1} p_{1} e_{n}\left(p_{2}\right)^{-1}$, где $e_{0}, e_{n}-Y$-ребра клеток $\pi_{1}$ и $\pi_{n}$, называемые торцами полосы, а края полосы $p_{1}, p_{2}$ составлены каждый как произведение по $i$ подпутей контуров клеток $\pi_{i}$, заключенных между их $Y$-ребрами. Края $Y$-полосы не содержат $Y$-ребер.

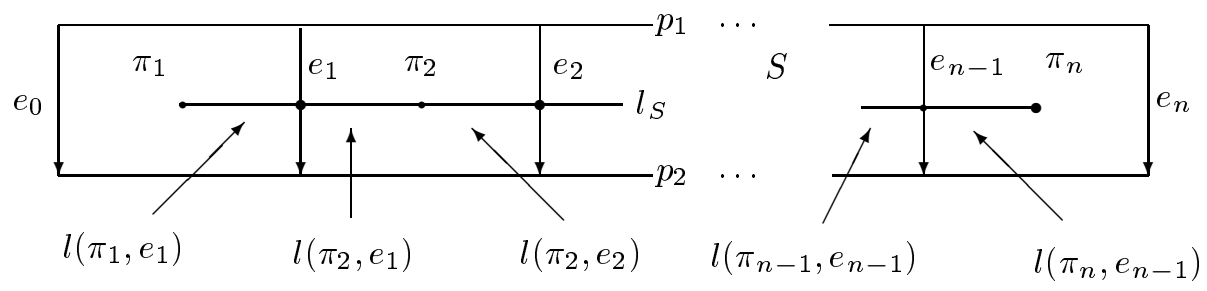

a

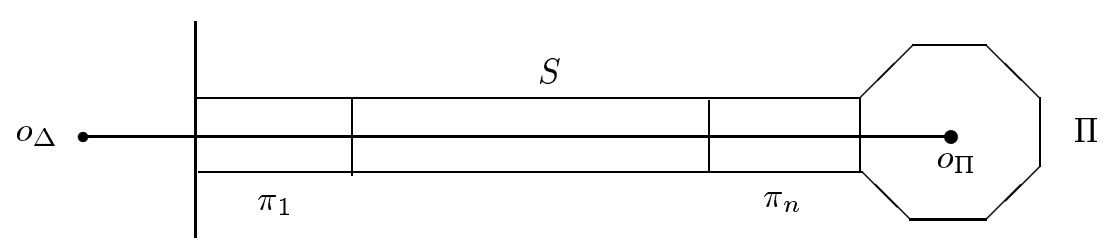

Рис. 4

Cредняя линия $l_{S}$ полосы $S$ составляется по определению из дуг $l\left(\pi_{1}, e_{1}\right)$, $l\left(\pi_{2}, e_{1}\right), l\left(\pi_{2}, e_{2}\right), \ldots, l\left(\pi_{n}, e_{n-1}\right)$ (рис. 4 a). В силу выбора множества соотношений $\mathscr{R}$ она не имеет самопересечений.

$Y$-полоса вида $\left[\pi_{1}, \ldots, \pi_{n}, \ldots, \pi_{n^{\prime}}\right]$ называется продолжением полосы $S$ вправо. Аналогично определяется продолжение полосы влево. Полоса, не имеющая продолжений, назьвается максимальной. Очевидно, что полоса $S$ максимальна, если или ее торцевые ребра совпадают, т.е. получается $Y$-кольцо (среднюю линию в этом случае тоже естественно замкнуть), или каждое из ее торцевых ребер лежит на границе диаграммы $\Delta$, либо лежит на границе некоторой клетки П, содержащей $Y$-ребро, но не отвечаюшей ни одному из соотношений выделенного множества $\mathscr{R}$. Такие клетки будем называть терминальными для $Y$-полос. В последнем случае 
продолжения средней линии максимальной полосы $S$ за торцевые ребра до точек $o_{\Delta}$ или $o_{\Pi}$ (рис. 4б) назовем медианой максимальной полосы $S$. Медианы можно строить и в случае максимальной полосы без клеток, когда $e_{0}=e_{n}$.

Если некоторая клетка $\pi$ входит в $Y$-полосу и в $Z$-полосу, причем пара $Y$-ребер циклически разделяет в $\partial \pi$ пару $Z$-ребер, то эти полосы пересекаются по определению. (Их средние линии имеют общую точку $o_{\pi}$. )

Перечислим типы полос в диаграммах над группой $B_{6}$ из $\S 4$ (а также группами $\left.B_{2}-B_{5}\right)$, которые встретятся в дальнейшем.

1. По определению в $\sigma$-полосу ( $\sigma$-кольцо) диаграммы $\Delta$ над группой $B_{6}$ разрешается включать любые $\sigma$-клетки. Они отвечают соотношениям (4.17)-(4.20). Терминальных клеток для $\sigma$-полос нет.

2. $d$-полоса ( $d$-кольцо) составляется по определению из $\left(d, k_{1}\right)$-клеток и $\left(d, a_{i}\right)$-клеток. (См. $\left.(4.15),(4.16).\right) \quad$ Терминальными клетками для $d$-полос служат $\left(\sigma, t_{1}\right)$-клетки. (См. (4.17).)

3. $b_{i}$-полосы (кольца) составляем из $\left(b_{i}, u\right)-,\left(b_{i}, a_{j}\right)$ - и $\left(b_{i}, k_{1}\right)$-клеток. (См. (4.12)-(4.14).) Терминальными для $b$-полос являются $(d, a)$-клетки. (См. (4.16).)

4. $k_{j}$-полоса (кольцо) составляется из $\left(k_{j}, r\right)-,\left(k_{j}, x\right)-,\left(k_{j}, \sigma\right)$-клеток и при $j=1$ из $\left(k_{1}, d\right)$-клеток (См. $(4.8),(4.9),(4.19)$ и (4.15).) Терминальньми для $k$-полос могут быть $(k, t)$-клетки, отвечающие соотношениям (4.10) (или втулки (hubs) в терминологии [8]), а также обобщающие их диски, вводимые в $\S 7$, которые также являются $(k, t)$-клетками.

5. $t_{j}$-полоса (кольцо) составляется из $\left(t_{j}, r\right)$ -,$\left(t_{j}, x\right)$ - и $\left(t_{j}, \sigma\right)$-клеток. (См. (4.6), $(4.7),(4.17)$ и (4.18).) Терминальньми для $t$-полос служат $(k, t)$-клетки.

Максимальные $k$ - и $t$-полосы, хотя бы один конец медианы которых находится внутри $(k, t)$-клетки (т.е. внутри втулки или, начиная с $\S 7$, внутри диска) назовем $k$ - и $t$-спицами.

6. $r_{i}$-полоса (кольцо) может состоять из $\left(r_{i}, t\right)-,\left(r_{i}, k\right)-\left(r_{i}, Q\right)-,\left(r_{i}, s_{\beta}, x\right)$ - и $\left(r_{i}, h, x\right)$-клеток. (См. (4.6), (4.8), (4.5), (4.3) и (4.4).) Терминальных клеток для $r$-полос не существует.

7. $Q$-полосу (кольцо) составляем только из $(Q, r)$-клеток. (См. (4.5).) В этом случае в отличие от остальных, определение $Y$-полосы используется не для одной буквы, а для множества $Y=\left\{q, q_{0}, \ldots, q_{n}\right\}$. Терминальньми для $Q$-полос могут быть $(\sigma, t)$-клетки $(4.17),(4.18)$ и $(k, t, Q)$-клетки $(4.10)$, а начиная с $\S 7,-$ диски, которые тоже являются $(k, t, Q)$-клетками.

8. $s_{\beta}$-полосы и кольца (в частности, $a_{i}$-полосы) составляются по определению из $\left(s_{\beta}, x\right)$ - и $\left(s_{\beta}, r, x\right)$-клеток (но не $\left(r, Q, s_{\beta}\right)$-клеток!), - см. $(4.2),(4.3),-$ а если $s_{\beta}=a_{i}$, то еще из $\left(a_{i}, \sigma\right)$-, $\left(a_{i}, d\right)$ - и $\left(a_{i}, b\right)$-клеток. (См. $(4.20),(4.16)$ и $\left.(4.13).\right)$ Терминальньми для $s$-полос служат $(r, Q)$-клетки $(4.5)$, а также, начиная с $\S 7$, диски.

9. $h$-полосы и кольца составляются из $(h, x)$ - и $(h, r, x)$-клеток. (См. $(4.2),(4.4)$. Терминальньми могут служить клетки, отвечающие соотношениям (4.17)-(4.19), (4.15), (4.14), (4.5), а также диски, вводимые в $\S 7$.

Наряду с $Y$-кольцами встретятся кольца, составленные из двух полос разных типов. Именно, рассмотрим некоторую $Y$-полосу $S=\left[\Pi_{1}, \pi_{1}, \ldots, \pi_{n}, \Pi_{2}\right]$ и $Z$-полосу $S^{\prime}=\left[\Pi_{1}, \pi_{1}^{\prime}, \ldots, \pi_{n^{\prime}}^{\prime}, \Pi_{2}\right]$ при $Z \cap Y=\varnothing$, имеющие в точности две общие клетки $\Pi_{1}$ и $\Pi_{2}$, причем все четыре торца этих полос лежат вне области $O$, ограни- 
ченной простой замкнутой кривой $x$, составленной из двух средних линий полос $S$ и $S^{\prime}$ (рис. 5а). Скажем в этом случае, что полосы $S$ и $S^{\prime}$ составляют $(Y, Z)$-кольцо $T$ с угловыми клетками $\Pi_{1}$ и $\Pi_{2}$ и с медианой $x$. Полосы $S$ и $S^{\prime}$ будем называть $Y$ и $Z$-частями $(Y, Z)$-кольца $T$.

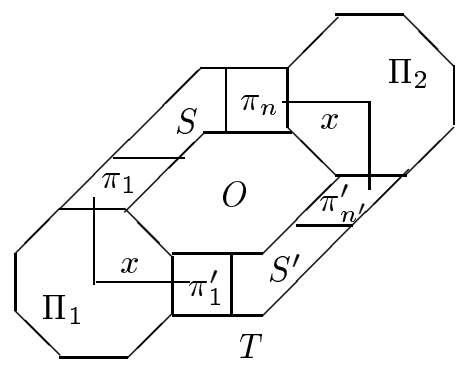

a

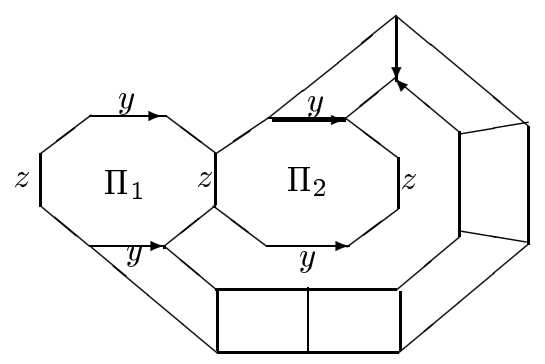

б

Рис. 5

Лемма 5.2. Пусть определяющее слово $R_{0}$ чиклически несократимо и имеет вид $z_{1}^{\varepsilon_{1}} W_{1} y W_{2} z_{2}^{\varepsilon_{2}} W_{3} y^{-1} W_{4}$, где буквиц $z_{1}, z_{2}, y$ не входят в слова $W_{1}, \ldots, W_{4}, \varepsilon_{1}, \varepsilon_{2}= \pm 1$ и $z_{1}^{\varepsilon_{1}} \neq z_{2}^{\varepsilon_{2}}$. Допустим, что в некоторой диаграмме $\Delta$ две клетки $\Pi_{1}$ и $\Pi_{2}$, отвечают определяющему слову $R_{0}$ и являются угловыми в некотором $(Y, Z)$-кольче (әде $\left.y \in Y, z_{1}, z_{2} \in Z\right), Z$-часть которого состоит только из двух клеток $\Pi_{1}$ и $\Pi_{2}$ (т.е. $\Pi_{1}$ и $\Pi_{2}$ имеют общее $Z$-ребро е). Тогда диаграмма $\Delta$ не является минимальной.

ДокАЗАТЕЛЬство. Рассмотрим два варианта приклеивания клетки $\Pi_{2}$ к $\Pi_{1}$ в диаграмме $\Delta$.

В первом из них одно и то же слово $V$, равное слову $R_{0}$ или его циклической перестановке, читается при обходе контуров клеток $\Pi_{1}$ и $\Pi_{2}$ в противоположных направлениях, начиная с ребра $e$. Но тогда диаграмма $\Delta$ не минимальна по лемме 5.1 (с пустым словом $U$ ).

Второй же вариант, когда слова, читаемые вдоль $\partial \Pi_{1}$ и $\partial \Pi_{2}$, начиная с $e$, различны, противоречит ориентируемости евклидовой плоскости и свойству $(Y, Z)$-кольца иметь все четыре торцевых ребра $Y$ - и $Z$-частей вне области, ограниченной медианой $x$ кольца (т.е. ситуация на рис. $5 б$ невозможна).

Кольцо $S_{1}$ считается меншше кольца $S_{2}$ в некоторой диаграмме, если область $O\left(S_{1}\right)$, ограниченная медианой кольца $S_{1}$, строго содержится в области $O\left(S_{2}\right)$, ограниченной медианой кольца $S_{2}$. Из конечности диаграммы $\Delta$ следует, что введенное частичное упорядочение колец в $\Delta$ является индуктивным.

Лемма Жордана и рассмотрение медиан колец делает очевидными следуюшие утверждения.

Лемма 5.3. Пусть в диаграмме $\Delta$ имеется $Y$-кольцо $S$, и $O(S)$ - область, ограниченная его медианой. Тогда

(1) если $S$ содержит клетку П, в которой два Y-ребра чиклически разделень парой Z-ребер, если этим же свойством обладают все 
$(Y, Z)$-клетки из $S$, а в $S$ и в $O(S)$ нет $Z$-терминальных клеток, то в $\Delta$ имеется $(Y, Z)$-кольцо, которое меньше кольца $S$ (рис. 6а);

(2) если в $O(S)$ нет $X$-терминальных клеток, а кольцо $S$ не содержит $X$-клеток, то либо в $O(S)$ нет $X$-клеток, либо есть $X$-кольцо.

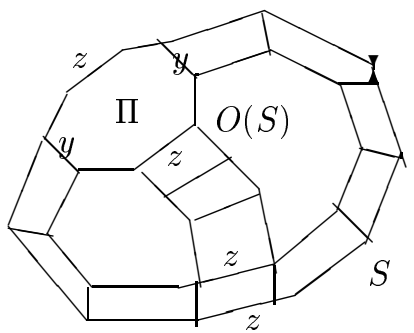

a

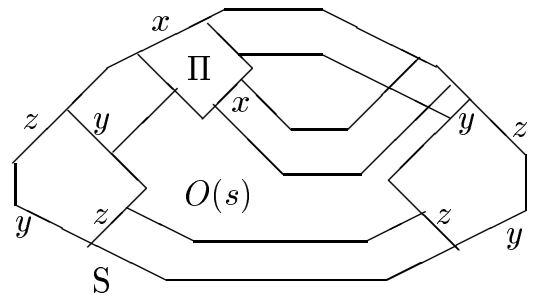

б

Рис. 6

Лемма 5.4. Пусть $S$ - $(Y, Z)$-кольцо в диаграмме $\Delta$, a $O(S)$ - область, ограниченная его медианой. Тогда

(1) если его $Y$-часть содержит неугловую клетку П, в которой два $Y$-ребра чиклически разделень парой $X$-ребер, если этим же свойством обладают все $(X, Y)$-клетки из $Y$-части, а в $S$ и в $O(S)$ нет $X$-терминальных клеток, то либо $Z \neq X$ и в $Z$-части кольиа $S$ есть $X$-клетки, либо в диаграмме есть $(X, Y)$-кольцо, которое меньше кольца $S$ (рис. 6б);

(2) если в $O(S)$ нет $X$-терминальных клеток, а кольцо $S$ не содержит $X$-клеток, то в $O(S)$ либо нет $X$-клеток, либо есть $X$-кольцо.

\section{§6. Отсутствие колец в диаграммах без $(k, t)$-клеток}

Невозможность многократного пересечения двух полос в минимальных диаграммах без $(k, t)$-клеток базируется ниже, как и в статье [8], на доказательстве отсутствия колец различных типов в таких диаграммах. Доказательство леммы 6.1 построено таким образом, что в индуктивном рассуждении предполагается выбранным минимальный контрпример к одному из ее утверждений, а значит, все утверждения можно считать справедливыми для меньших колец, т.е. меньших колец поименованных видов не существует. Все перекрестные ссылки в доказательстве серии утверждений леммы 6.1 носят именно такой характер, и мы не будем повторять каждьй раз, что такие ссылки корректны, поскольку используются индуктивные предположения. При рассмотрении полос того или иного вида нужно помнить, из клеток какого типа эти полосы составляются. Для этого полезно иметь под рукой перечень $1-9$ из $§ 5$.

Лемма 6.1. Пусть $\Delta$ - минимальная диаграмма без $(k, t)$-клеток над копредставлением группы $B_{6}$ из $\S 4$, т.е. в $\Delta$ могут входить только клетки, 
отвечающие соотношениям (4.2)-(4.9) и (4.11)-(4.20), но не (4.10). Тогда в $\Delta$ Hem

(1) б-колеи;

(2)

(3) $(r, Q)$-колеи;

(4) $Q$-колеи;

(5) $(r, t)$-колеи;

(6) $(r, k)$-колеи;

(7) $t$-колеи;

(8) k-колеи;

(9) $(\sigma, k)$-колеи;

(10) $(\sigma, t)$-колеи;

(11) $(d, k)$-колеи;

(12) (b, a)-колеи, без $\sigma$ - и d-клеток;

(13) $(b, k)$-колеи без $\sigma$ - и d-клеток;

(14) $(d, a)$-колеи без $t$ - и $Q$-клеток;

(15) $(\sigma, a)$-колеи;

(16) $(r, s)$-колеи без $Q$-клеток;

(17) $(r, h)$-колеи без $Q$-клеток;

(18) d-колеч;

(19) в-колеи;

(20) а-колеи, содержащих $(d, a)$-клетки.

ДокАЗАТЕльство. Доказывая утверждения леммы "от противного", мы выбираем среди всех колец, являющихся контрпримерами к одному из утверждений (1)-(20), минимальное кольцо $S$. Через $\Delta(S)$ обозначаем поддиаграмму, составленную из всех клеток кольца $S$ и всех клеток, находящихся в области $O(S)$, ограниченной медианой кольца $S$.

(1) Допустим, что $S$ является $\sigma$-кольцом и в нем есть $(\sigma, k)$-клетка. Поскольку в этой клетке пара $\sigma$-ребер циклически разделяет пару $k$-ребер, а в диаграмме без $(k, t)$-клеток нет $k$-терминальных клеток, в диаграмме $\Delta$ есть $(\sigma, k)$-кольцо меньшее, чем $S$, по лемме 5.3(1). Но это противоречит свойству (9) и минимальности выбранного контрпримера $S$. Аналогично, наличие в $S(\sigma, t)$-клетки давало бы меньший контрпример к утверждению (10).

Значит, $S$ состоит только из $(\sigma, a)$-клеток, и метка внешнего края кольца совпадает с внутренней. (См. (4.20).) В этом случае можно отождествить два края после удаления внутренности кольца $S$ из $\Delta$. Полученная диаграмма имеет меньший тип, вопреки минимальности диаграммы $\Delta$.

(2) Допустим, что $S$ - это $r$-кольцо. Наличие в $S t$-клеток противоречило бы по лемме 5.3(1) и свойству (5) минимальности контрпримера $S$. Значит, по свойству $(7)$ и лемме $5.3(2) t$-клеток нет и в $\Delta(S)$, т.е. нет терминальных клеток для $Q$-полос. Поэтому в $S$ нет $Q$-клеток по лемме $5.3(1)$ и свойству (3), т.е. нет терминальных клеток для $s$ - и $h$-колец. Следовательно, по утверждениям (16) и $(17)$ в $S$ не может быть и $\left(r, s_{\beta}, x\right)$ - или $(r, h, x)$-клеток, т.е. в $S$ нет никаких клеток.

(3) Предположим, что $S$ - это $(r, Q)$-кольцо. Поскольку его $Q$-часть $T$ может состоять только из $r$-клеток, в $T$ нет неугловых клеток, так как в противном случае 
по лемме 5.4(1) можно было бы найти меньшее, чем $S(r, Q)$-кольцо. В таком случае угловые клетки склеены в $T$ по общему $Q$-ребру, и отвечают одному и тому же соотношению вида (4.5), так как входят в одну $r_{i}$-полосу. При $q_{i_{1}} \neq q_{i_{2}}$ эти клетки, очевидно, являются зеркальными копиями вопреки минимальности диаграммы, а при $q_{i_{1}} \equiv q_{i_{2}}$ получается противоречие с утверждением леммы 5.2 , если принять $Y=\left\{r_{i}\right\}$, а $Z=Q$.

(4) Предположим, что $S-Q$-кольцо. Поскольку оно состоит лишш из $(r, Q)$-клеток, лемма 5.3(1) и утверждение (3) приводят к противоречию с минимальностью контрпримера.

$(5)$ Допустим, что $S-(r, t)$-кольцо. Тогда в его $t$-части $T$ нет $\sigma$-клеток по лемме 5.4(1) и свойству (10) для меньших колец. Аналогично, по свойству (5) для меньших колец, среди неугловых клеток в $T$ нет $(r, t)$-клеток, т.е. они могут быть лишь $(t, x)$-клетками, причем их приклеивание вдоль $t$-ребер не может быть зеркальньм по лемме 5.1. Таким образом, на каждом из краев $p$ полосы $T^{\prime}$, полученной из $T$ отбрасыванием угловых клеток, написано слово вида $x^{ \pm n}$, где $n$ - число клеток в $T^{\prime}$.

В $\Delta(S)$ нет $\sigma$-клеток по лемме $5.4(2)$ и свойству (1), так как их не может быть в $S$, т.е. нет терминальных клеток для $Q$-полос.

Снова по лемме 5.4(1) среди неугловых клеток $r$-части полосы $S$ нет $Q$-, $t$ - и $k$-клеток по свойствам $(3),(5)$ и (6) для меньших колец. Поэтому они могут быть только $(r, s, x)$ - или $(r, h, x)$-клетками, а на крае $\bar{p} r$-части после отбрасывания угловых клеток читается слово $V$ от $h x$ и $s_{\beta} x, \beta=0, \ldots, M$ (или от $h x^{-1}$ и $s_{\beta} x^{-1}$ ).

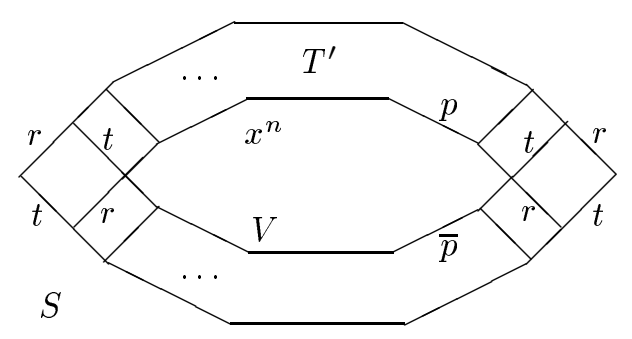

Рис. 7

Сравнивая теперь метки краев $p$ и $\bar{p}$ с общими началом и концом (рис. 7 ), получаем по лемме ван Кампена равенство

$$
x^{n}=V\left(h x, s_{0} x, \ldots, s_{M} x\right)
$$

в силу соотношений, отвечающим клеткам, находящимся в области $O(S)$. (Второй вариант с $h x^{-1}$ и т. д. может быть рассмотрен вполне аналогично.)

Поскольку буквы $\sigma, t_{j}, k_{j}$ не входят в равенство $(6.1)$, а в $O(S)$ не может быть $\sigma$-, $t$ - или $k$-колец по свойствам $(1),(7)$ и $(8)$, там нет и $\sigma$-, $t$ - или $k$-клеток. В частности, нет терминальных клеток для $d$-полос, а следовательно, нет и $d$-клеток по свойству (18). Поэтому нет терминальных клеток для $b$-полос, а значит, нет и $b$-клеток по свойству (19). Наконец в $O(S)$ нет $(u, u)$-клеток, так как с ними могут граничить только $(u, u)$ - и $(b, u)$-клетки. 
Таким образом, равенство (6.1) выполнено уже в группе $B_{2}$. По лемме 4.3 это возможно только при $n=0$. Это значит, что $t$-часть $T$ состоит всего из двух угловых клеток. Их метки должны быть одинаковы, ибо они еще входят в одну $r_{i}$-полосу. В этом случае лемма 5.2 дает противоречие с минимальностью диаграммы $\Delta$.

(6) Допустим, что $S-(r, k)$-кольцо. В этом случае противоречие получается так же, как в предыдушем случае, с заменой ссылок на свойства (10) и (5) ссылками на (9) и (6). В случае $k=k_{1} b$-клетки в $k$-части кольца $S$ исключаются после исключения $\sigma$ и $d$-клеток из $\Delta(S)$ с учетом леммы $5.4(1)$ и свойства (13) для меньших колец.

(7) Допустим, что $S-t$-кольцо. Тогда по лемме $5.3(1)$ и свойству $(10)$ в $S$ нет $\sigma$-клеток, а по свойству (5) - нет $r$-клеток. Это означает, что в $S$ входят лишь $(t, x)$-клетки, а внешний край кольца $S$ имеет ту же метку, что и внутренний: $x^{n}$. В этом случае можно отождествить два края после вырезания кольца $S$ из $\Delta$. Полученная диаграмма имеет меньший тип вопреки минимальности диаграммы $\Delta$.

(8) Предположим, что $S-k$-кольцо. Тогда, последовательно применяя свойства $(9),(6),(11),(18),(13)$ и лемму 5.3 , выясняем, что в $S$ нет $\sigma-, r-, d-$ и $b$-клеток, т.е. оно состоит из $(k, x)$-клеток. Отсюда выводится противоречие, как и в пункте $(7)$.

(9) Допустим, что $S-(k, \sigma)$-кольцо. По свойствам $(10),(7),(2)$ и (6) для меньших колец и по лемме 5.4 в $\Delta(S)$ нет $t$ - и $r$-клеток. В частности, нет терминальных клеток для $a$-полос. Значит, и $a$-клеток нет в $\sigma$-части по свойству (15) и лемме 5.4(1). По свойству (9) и лемме $5.4(1) k$-клетки присутствуют только в $k$-части кольца $S$. Таким образом, в $\sigma$-части кольца $S$ имеются только угловые клетки. Эти клетки имеют одинаковые метки, так как входят также в одно $k$-кольцо. Но тогда к противоречию ведет ссылка на лемму 5.2 .

(10) В случае, когда $S-(t, \sigma)$-кольцо, рассуждение вполне аналогично предыдущему (с заменой “ $k$ ” на “ $t ”$, а свойства (6) на свойство (5)).

(11) Допустим, что $S$ - это $(d, k)$-кольцо. Тогда в нем нет $\sigma$-клеток по свойству (9) для меньших колеци лемме $5.4(1)$, а по свойству (1) и лемме $5.4(2)$ в $\Delta(S)$ тоже нет $\sigma$-клеток. В этом случае по свойству (11) для меньших колеци лемме 5.4(1) в $d$-части кольца $S$ нет неугловых $k$-клеток.

Поскольку в $\Delta(S)$ нет $Q$-терминальных клеток, по лемме 5.4 и свойству (4) в этой поддиаграмме совсем нет $Q$-клеток, в частности, нет и $a$-терминальных клеток. По свойству $(7)$ и лемме $5.4(2)$ в $\Delta(S)$ отсутствуют $t$-клетки. В таком случае лемма 5.3(1) вместе со свойством (14) означают отсутствие неугловых $(d, a)$-клеток в $S$.

Таким образом, в $d$-части кольца $S$ нет неугловых клеток, что стандартным уже образом ведет к противоречию с леммой 5.2.

(12) Допустим, что $S$ - это $(a, b)$-кольцо без $\sigma$ - и $d$-клеток. По свойству $(1)$ и лемме $5.4(2) \sigma$-клеток нет и в поддиаграмме $\Delta(S)$. Тогда по свойству $(18)$ в $\Delta(S)$ нет и $d$-клеток, в частности, нет терминальных клеток для $b$-полос. По лемме $5.4(2)$ и свойству $(7)$ в $\Delta(S)$ нет $t$-клеток, т.е. нет $Q$-терминальных клеток, а значит, совсем нет $Q$-клеток по свойству (4). Поэтому нет и $a$-терминальных клеток.

В таком случае по свойствам (12) и (13) и лемме 5.4(1) среди неугловых клеток $b$-части кольца $S$ нет ни $a$-, ни $k$-клеток. Они могут быть лишь $(b, u)$-клетками.

В кольце $S$ не может быть $r$-клеток по лемме $5.3(1)$ и свойству $(16)$, ибо $a_{j} \in$ 
$\left\{s_{0}, \ldots, s_{M}\right\}$. В $a$-части неугловыми могут быть только $(a, x)$-клетки, так как клетки других типов исключаются тем же способом, как и из $b$-части.

Сравнивая выводы, сделанные относительно меток краев $a$ - и $b$-частей, приходим, как и в пункте (5), к равенству вида

$$
x^{n}=U \text {, }
$$

где $n$ - число (или удвоенное число) неугловых клеток в $a$-части кольца $S$, а $U=U\left(u_{1}, \ldots, u_{m}\right)$. При этом соотношение (6.2) вьполняется уже в группе $B_{1} * R_{u}$, так как $b$-клетки тоже удаляются из диаграммы равенства (6.2) с помощью свойства (12). Но в свободном произведении равенство (6.2) возможно по лемме 4.2 лишь при $n=0$.

Таким образом, в $a$-части кольца $S$ нет неугловых клеток, что, как обычно, ведет к противоречию с леммой 5.2 .

(13) Пусть $S-(b, k)$-кольцо без $\sigma$ - и $d$-клеток. Тогда таких клеток нет и в $\Delta(S)$, как в пункте (12). В $b$-части кольца $S$ нет неугловых $k$-клеток по лемме 5.4(1) и свойству (13) для меньших колец.

По свойствам (6), (2) и лемме 5.4 в $\Delta(S)$ нет $r$-клеток, а значит, нет $a$-терминальных клеток. Тогда в $b$-части кольца $S$ нет $a$-клеток по свойству (12).

Таким образом, неугловые клети в $b$-части кольца могут быть лишш $(b, u)$-клетками, после чего, как и в пункте (12), получается равенство вида (6.2), приводяшее к противоречию.

(14) Пусть $S$ - это $(d, a)$-кольцо без $t$ - и $Q$-клеток. По лемме 5.4 и свойствам (16) и (2) в поддиаграмме $\Delta(S)$ нет $r$-клеток, по свойствам (15) и (1) в ней нет $\sigma$-клеток, а по свойствам (11) и (8) - нет $k$-клеток. По свойству (4) и лемме $5.4(2)$ в $\Delta(S)$ нет $Q$-клеток, т.е. $a$-терминальных клеток. Поэтому по утверждению (14) и лемме $5.4(1)$ в $d$-части кольца $S$ нет неугловых $(d, a)$-клеток. Приходим к отсутствию неугловых клеток вообше в $d$-части. Остается стандартным образом применить лемму 5.2 .

(15) Допустим, что $S$ - это $(\sigma, a)$-кольцо. По свойствам (16) и (2) для менших колец и лемме 5.4 в поддиаграмме $\Delta(S)$ нет $r$-клеток, т.е. нет $a$-терминальных клеток. Поэтому по лемме 5.4(1) и свойству (15) неугловые клетки $\sigma$-части $T$ кольца $S$ не могут быть $a$-клетками.

По свойствам (9), (10) и лемме 5.4(1) в $S$ нет также $k$ - и $t$-клеток. Приходим к выводу, что в $T$ есть только угловые клетки, что опять приводит к противоречию с леммой 5.2 .

(16) Допустим, что $S$ - это $(r, s)$-кольцо без $Q$-клеток. В поддиаграмме $\Delta(S)$ нет $t$-клеток по лемме 5.4 и утверждениям (5) и (7) и нет $k$-клеток по свойствам $(6)$ и (8). Отсюда следует, что в $\Delta(S)$ нет $Q$-клеток по свойствам (3) и (4), т.е. нет $s$-терминальных клеток. В $r$-части $T$ нет неугловых $s$-клеток по свойству (16) и лемме 5.3(1). В итоге, $T$ не содержит никаких неугловых клеток, что снова ведет к противоречию с утверждением леммы 5.2.

(17) Пример $(r, h)$-кольца разбирается точно так же, как предыдуший.

(18) Допустим, что $S$ - это $d$-кольцо. По лемме 5.3 и свойству (1) в поддиаграмме $\Delta(S)$ нет $\sigma$-клеток, а по свойствам $(11),(8)$ и лемме 5.3 в $S$ нет $k$-клеток. 
По свойствам (7) и (2) и лемме 5.3(2) в $\Delta(S)$ нет $t$ - и $Q$-клеток, т.е. терминальных клеток для $a$-полос. Значит, по свойству $(14)$ в $S$ нет и $(d, a)$-клеток. Другими словами, в $S$ нет вообще никаких клеток.

(19) Предположим, что $S-b$-кольцо. В поддиаграмме $\Delta(S)$ нет $\sigma$-клеток по лемме 5.3(2) и свойству (1), т.е. нет $d$-терминальных клеток. В таком случае по свойству (18) и той же лемме в $\Delta(S)$ нет и $d$-клеток. После этого $k$-клетки исключаются из $S$ с помощью свойства (13).

В $\Delta$ нет $r$-клеток по свойству (2) и лемме $5.3(2)$, т.е. нет $a$-терминальных клеток, что позволяет вообще исключить $a$-клетки из $S$ с помощью свойства (12). Значит, в $S$ остаются только $(b, u)$-клетки, а на внешнем и на внутреннем краях этого кольца написаны одинаковые слова. Последнее противоречит минимальности диаграммы $\Delta$, как и в пункте $(7)$.

(20) Пусть $S-a$-кольцо с $(d, a)$-клеткой. По свойствам (1), $(15)$ и лемме 5.3 в $\Delta(S)$ нет $\sigma$-клеток, т.е. нет терминальных клеток для $d$-полос. Кроме того, по свойству $(7)$ в $\Delta$ нет $t$-клеток. Тогда по свойству (4) нет и $Q$-клеток, ибо нет терминальных клеток для $Q$-полос. Поэтому наличие в $S(d, a)$-клетки ведет к противоречию со свойством (14) для меньшей полосы.

Таким образом, контрпримера к утверждению леммы 6.1 не существует.

\section{§7. Диаграммы над группами $B_{2}, C$ и $B$}

Лемма 7.1. Пусть $L_{1}, L_{2}$ - приведенные слова от $x, r_{i}, i \in I$, а $W_{1}, W_{2}$ приведенное слова от $q, q_{0}, \ldots, q_{N}, s_{0}, \ldots, s_{M}, h$, причем в $W_{2}$ имеется всего одно вхождение $Q$-буквы $q_{j} \in\left\{q, q_{0}, \ldots, q_{N}\right\}$. Тогда из равенства $L_{1} W_{1} L_{2}=$ $W_{2}$ в группе $B_{2}$ следует, что слово $W_{1}$ содержит лишь одно вхохдение Q-букввь.

ДокАЗАТЕЛЬСтво. Рассмотрим минимальную диаграмму над $B_{2}$ с контуром $p_{1} p_{2} p_{3} p_{4}$, где $\varphi\left(p_{1}\right) \equiv L_{1}, \varphi\left(p_{2}\right) \equiv W_{1}, \varphi\left(p_{3}\right) \equiv L_{2}, \varphi\left(p_{4}\right) \equiv W_{2}^{-1}$.

Если бы путь $p_{2}$ не был простым, то между написанными на нем буквами возникло бы нетривиальное соотношение вопреки лемме 4.2 .

Если в $p_{2}$ имелось хотя бы два $Q$-ребра, то неизбежно возникла бы некоторая $Q$-полоса $S$ с торцами на пути $p_{2}$, так как путь $p_{3} p_{4} p_{1}$ содержит лишь одно $Q$-ребро. Эта полоса не может быть без клеток ввиду предыдушего замечания. В то же время, наличие в ней $(Q, r)$-клетки неизбежно привело бы к образованию $(Q, r)$-кольца, ибо в пути $p_{2}$ нет $r$-ребер (рис. $\left.8 \mathrm{a}\right)$. Полученное противоречие с леммой 6.1(3) означает, что в пути $p_{2}$ не более одного $Q$-ребра.

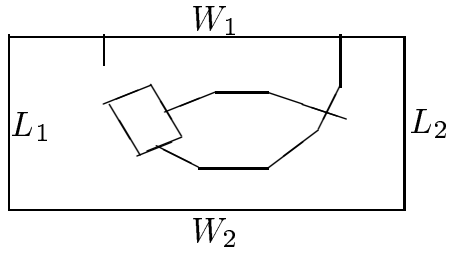

a

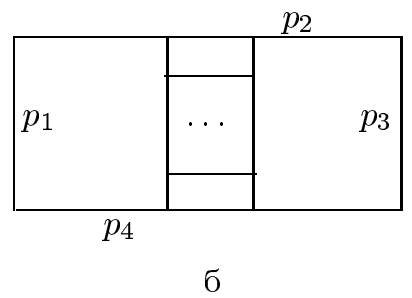

б

Рис. 8 
$Q$-ребро пути $p_{4}$ должно соединяться с каким-то ребром на $p_{2} Q$-полосой (или просто лежать на $p_{2}$, рис. $\left.8 б\right)$, и лемма доказана.

Лемма 7.2. Пусть диаграмма $\Delta$ над группой $B$ содержит две клетки $\Pi_{1}$ и $\Pi_{2}$, отвечающие соотношению (4.10), причем әти клетки обладают общими смежными (в каждой из клеток) спицами, соединяющими точки оп и $\Pi_{2}$, а в области О, ограниченной этими спицами, нет других спиц и других $(k, t)$-клеток. Тогда $\Delta$ не является минимальной диаграммой, а именно, число ее $(k, t)$-клеток можнно уменьиить на 2 при сохранении граничной метки.

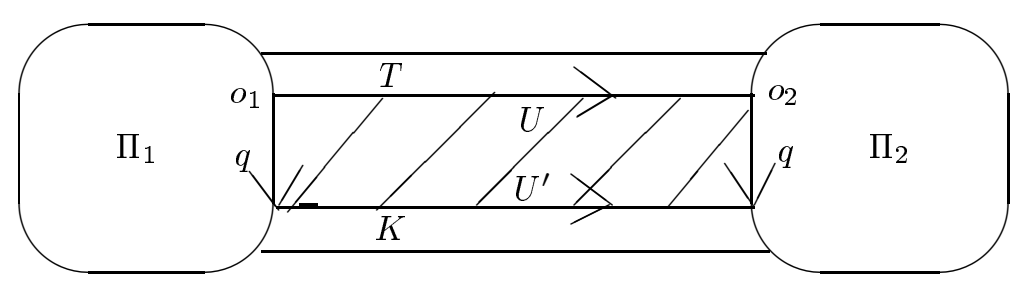

Рис. 9

ДокАЗАТЕЛьство. Итак, имеем две полосы: $t$-полосу $T$ и $k$-полосу $K$ с терминальными клетками $\Pi_{1}$ и $\Pi_{2}$, через которые проходят данные в условии спицы (рис. 9). Пусть $U$ - слово, написанное на крае $p$ полосы $T$, находящемся в области $O$, от клетки $\Pi_{1}$ до клетки $\Pi_{2}$. Аналогично определяется метка края $U^{\prime}$ полосы $K$. Поскольку спицы выбраны смежньми, получаем некоторую поддиаграмму (заштрихована на рис. 9) с граничной меткой $q^{-1} U q\left(U^{\prime}\right)^{-1}$, причем равенство

$$
U^{\prime}=q^{-1} U q
$$

выполнено уже в группе $C$, заданной по определению всеми определяющими соотношениями группы $B$, кроме соотношения (4.10), так как в $O$ нет $(k, t)$-клеток.

Отметим еще, что в группе $C$

$$
U t=t U, \quad U^{\prime} k=k U^{\prime},
$$

так как все $t$-клетки, входящие в $T$ (все $k$-клетки, входящие в $K$ ), отвечают соотношениям коммутации (4.6) и (4.7) (соответственно (4.8) и (4.9)).

Пусть $V$ - граничная метка клетки $\Pi_{1}$ (клетки $\Pi_{2}$ ), которую можно прочитать, начиная с вершины $p_{-}$(с $\left.p_{+}\right)$и $Q$-ребра. Она имеет вид $V \equiv V_{1} \cdots V_{2 L}$, где $V_{i} \equiv q k q^{-1} t$ для некоторых $k=k_{j}^{ \pm 1}, t=t_{l}^{ \pm 1}$. Ввиду (7.1) и (7.2)

$$
V_{i} U V_{i}^{-1}=q k q^{-1} t U t^{-1} q k^{-1} q^{-1}=q k U^{\prime} k^{-1} q^{-1}=q U^{\prime} q^{-1}=U
$$

в группе $C$, а значит, слово $U$ коммутирует со словом $V$ в группе $C$. Поэтому диаграмма $\Delta$ не является минимальной по лемме 5.1.

В дальнейших рассуждениях будет встречаться вспомогательньй граф $\Gamma(k, t)$, построенный по диаграмме $\Delta$. Его внутренними вершинами являются по определению точки $o_{\Pi}$, выбранные внутри $(k, t)$-клеток П диаграммы $\Delta$, а внешней - вершина $o_{\Delta}$. Все $k$ - и $t$-спицы считаются по определению ребрами графа $\Gamma(k, t)$. 
ЛЕмма 7.3. Естественный гомоморфизм $B_{2} \rightarrow B$ инбективен.

ДокАЗАТЕЛЬСтво. Рассуждая “от противного", рассмотрим минимальную диаграмму $\Delta$, на контуре которой написано неединичное в $B_{2}$ слово от порождающих этой группы.

Допустим сначала, что в $\Delta$ есть $(k, t)$-клетки и рассмотрим граф $\Gamma(k, t)$. В нем нет одноугольников, поскольку $t$-ребра в $(k, t)$-клетках циклически разделены $k$-ребрами. В нем нет и 2-угольников с внутренними вершинами по лемме 7.2. Значит, этот граф является $4 L$-графом и, во всяком случае, 8 -графом в терминологии $\S 3$, ибо $L>1$. По лемме 3.2 найдется спица, инцидентная вершине $o_{\Delta}$, а следовательно на $\partial \Delta$ есть $k$ - и $t$-ребра. Но буквы $k$ и $t$ не входят в число порождающих группы $B_{2}$. Таким образом, в $\Delta$ нет $(k, t)$-клеток.

В таком случае $\Delta$ - минимальная диаграмма надгруппой $C$, которая, очевидно, является HNN-расширением группы $B_{2}$ с проходными буквами $t_{1}, \ldots, k_{1}, \ldots$ Как известно, базовая подгрупша изоморфно вложена в $\mathrm{HNN}-$ расширение, а значит, рассматриваемая диаграмма $\Delta$ невозможна.

Лемма 12.13 [6] (где $L=1$ ) видоизменяется теперь следуюшим образом.

Лемма 7.4. Пусть $\Sigma-h$-специальное слово и

$$
\prod_{j=1}^{L} k_{j}^{-1} \Sigma^{-1} t_{j} \Sigma k_{j} \Sigma^{-1} t_{j}^{-1} \Sigma=1
$$

в группе B. Тогда в минимальной диаграмме $\Delta$ этого равенства содержится одна $(k, t)$-клетка, и существуют слова $L_{1}, L_{2}$ в алфавите $\left\{x, r_{i} ; i \in I\right\}$ такие, что $L_{1} \Sigma L_{2}=q$ в группе $B_{2}$.

ДокАЗАТЕльСТво. Если бы в $\Delta$ не было $(k, t)$-клеток, т.е. терминальных клеток для $k$ - и $t$-полос, то максимальные $k$ - и $t$-полосы пересекались бы, как видно из формы левой части в (7.3). Но клеток, входящих одновременно в $k$ - и $t$-полосу, не существует по определению таких полос, данному в $\S 5$.

Как было замечено при доказательстве леммы 7.3 , граф $\Gamma(k, t)$ является $l$-графом для $l=4 L$. Поэтому, число $(k, t)$-клеток в $\Delta$ не больше одной, ибо иначе по лемме 3.4 обшее число $k$ - и $t$-ребер в $\partial \Delta$ было бы не меньше $8 L-6$, в то время как их ровно $4 L$ (и $L>1$ ).

Спицы единственной $(k, t)$-клетки должны проходить через $4 L k$-и $t$-ребер в $\partial \Delta$, а в образовавшихся между спицами "секторах" будут поддиаграммы над группой $C$ с граничньми метками вида $L_{1} \Sigma L_{2} q^{-1}$ для слов $L_{1}$ и $L_{2}$, удовлетворяющих требованиям леммы, как видно из определения $k$ - и $t$-полос и того обстоятельства, что $\Delta$ является диаграммой над $B$. По лемме 7.3 из равенства $L_{1} \Sigma L_{2}=q$ в групше $C$ следует это же равенство в группе $B_{2}$.

Лемма 7.3 вместе с леммами 4.1, 4.4 и 4.5 дает следующий аналог леммы Буна $12.20[6]$.

ЛЕмма 7.5. Для h-специального слова $\Sigma$ равенство (7.3) выполнено в группе $B$ тогда и только тогда, когда $\Sigma^{*}=q$ в полугруппе $\Gamma(T)$. В частности, 
положительное слово $\boldsymbol{w}$ в алфавите $\left\{s_{0}, \ldots, s_{M}\right\}$ содержится в множестве $E$ (см. $§ 4)$ тогда и только тогда, когда слово

$$
\prod_{j=1}^{L} k_{j}^{-1}\left(h^{-1} q_{1} w h\right)^{-1} t_{j}\left(h^{-1} q_{1} w h\right) k_{j}\left(h^{-1} q_{1} w h\right)^{-1} t_{j}^{-1}\left(h^{-1} q_{1} w h\right)
$$

равно 1 в әруппе $B$.

Для последующего “выпрямления" $r$ - и $\sigma$ - полос удобно к определяющим соотношениям группы $B$ (а также групп $\left.B_{4}, B_{5}, B_{6}\right)$ добавить их следствия $(7.3)$ для всех $h$-специальных слов $\Sigma$ таких, что $\Sigma^{*}=q$ в $\Gamma(T)$, в частности, добавить определяюшие слова (7.4) для $w \in E$. Соотношения такого рода можно встретить в [6] (cм. plate 2, лемма 12.24), а их аналоги в [8] названы дисками.

Диском или $(k, t)$-клеткой будем далее называть любую клетку в диаграмме, отвечающую соотношению вида (7.3) или соотношению (4.10). Формально (но не содержательно) понятие минимальной диаграммы, данное в $\S 5$, сохраняется и после добавления дисков к списку клеток, которыми заполняются диаграммы. Можно говорить о спицах, выходящих из диска и т.п.

Лемма 7.6. Пусть диаграмма $\Delta$ над группой $B$ содержит два диска $D_{1}$ и $\mathrm{D}_{2}$ с общими смежсными спицами, причем в области О межсду этими спииами нет других дисков и нет других спиц. Тогда диаграмма $\Delta$ не является минимальной, т.е. при сохранении граничной метки число ее дисков можсно уменьиить на 2.

ДокАЗАТЕЛЬСтво. Каждый из дисков можно заменить по лемме 7.4 одной стандартной $(k, t)$-клеткой, отвечающей соотношению $(4.10)$, и клетками менњших рангов. Полученные вместо $D_{1}, D_{2}(k, t)$-клетки $\Pi_{1}, \Pi_{2}$ удовлетворяют условию леммы 7.2, из которой и выводится теперь утверждение леммы 7.6.

Лемма 7.7. Пусть $\Sigma \equiv q$ либо $\Sigma \equiv h^{-1} X^{\#} q_{j} Y h-h$-специальное слово, содержсащее для некоторого $i \in I$ вхождение слова $F_{i}^{\#} q_{i_{1}} G_{i}$ или вхожсдение слова $H_{i}^{\#} q_{i_{2}} K_{i}$, т.е. для некоторого соотношения (4.5) и некоторьх слов $U, V q_{j}=q_{i_{1}}, h X_{i} \equiv U F_{i}, Y_{i} h \equiv G_{i} V$ (соответственно $q_{j}=q_{i_{2}}$, $\left.h X_{i} \equiv U H_{i}, Y_{i} h \equiv K_{i} V\right)$. Тогда слово $W \equiv U^{\#} r_{i} H_{i}^{\#} q_{i_{2}} K_{i} r_{i}^{-1} V$ (слово $\left.U^{\#} r_{i}^{-1} F_{i}^{\#} q_{i_{1}} G_{i} r_{i} V\right)$, полученное из $\Sigma$ с помощью однократного применения соотношения (4.5), равно в ранге меньшем, чем ранг соотношений (4.5), слову вида $x^{n_{1}} r_{i} x^{n_{2}} \Sigma_{1} x^{n_{3}} r_{i}^{-1} x^{n_{4}}$ (вида $\left.x^{n_{1}} r_{i}^{-1} x^{n_{2}} \Sigma_{1} x^{n_{3}} r_{i} x^{n_{4}}\right)$, әде $\Sigma_{1}$ является h-спечиальным словом или $\Sigma_{1} \equiv q$.

ДокАЗАТЕльство. Случай $\Sigma \equiv q$ очевиден $\left(\right.$ с $\left.n_{1}=\cdots=n_{4}\right)$ ввиду единственности соотношения вида $r_{i_{0}}^{-1} h^{-1} q_{0} h r_{i_{0}}=q$ среди соотношений (4.5). Только это соотношение содержит $h^{-1} q_{0} h$, что делает очевидным и случай $i=i_{0}$.

В любом другом случае $\Sigma$ - $h$-специальное слово, а после применения соотношения (4.5) опять получится слово с началом $h^{-1}$ и концом $h$, в котором нет других вхождений $h^{ \pm 1}$, по определению соотношений (4.5).

Рассмотрим далее только первый вариант. Для положительного слова $T$ от $s_{0}, \ldots, s_{M}, h$ обозначим через $T_{x}$ (через $T_{-x}$ ) результат замены каждой буквы $s_{j}, h$ на $s_{j} x, h x\left(\right.$ на $\left.s_{j} x^{-1}, h x^{-1}\right)$. 
В силу соотношений (4.2) и положительности слова $V$ подслово $V$ слова $W$ равно слову вида $V_{x} x^{n_{4}}$ для некоторого показателя $n_{4}$. После такой замены в слове $W$ подслово $r_{i}^{-1} V_{x}$ заменим равным ему в силу соотношений (4.3) словом $V_{-x} r_{i}^{-1}$. Затем подслово $V_{-x}$ с помошью соотношений (4.2) можно заменить на слово вида $V x^{n_{3}}$ опять-таки ввиду положительности слова $V$.

После аналогичной переработки подслова слова $W$, находящегося левее буквы $q_{i_{2}}$, получается требуемый результат.

Вхождение в слово $\Sigma$, указанное для некоторого $i \in I$ в условии леммы 7.7 , назовем для краткости $i$-вхождением.

Рассмотрим следуюшую вспомогательную конструкцию. Пусть $D$ - диск в некоторой минимальной диаграмме $\Delta$ над группой $B$, а $S$ - некоторая $r_{i}$-полоса в $\Delta$, последовательно пересекающая $t$-полосу $T$ и $k$-полосу $K$, определяемые смежными $t$ - и $k$-спицами диска $D$, которые пересекают среднюю линию полосы $S$ ровно в одной точке $o_{\Pi_{1}}, o_{\Pi_{2}}$ каждая (рис. $\left.10 \mathrm{a}\right)$. Обозначим через $\Delta(S, T, K)$ поддиаграмму, составленную из клеток полос $T, K$ (идущих от диска $D$ до клеток $\Pi_{1}, \Pi_{2}$ включительно), клеток полосы $S$ (от $\Pi_{1}$ до П 2 включительно) и всех клеток, целиком лежащих в области $O(S, T, K)$, ограниченной указанньми спицами и средней линией полосы $S$. (Она заштрихована на рис. 10а.)

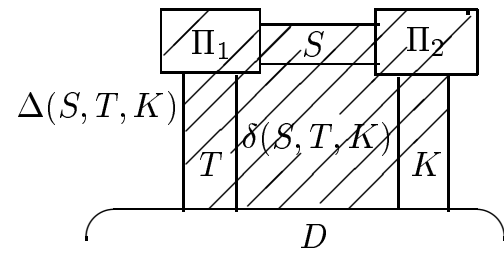

a

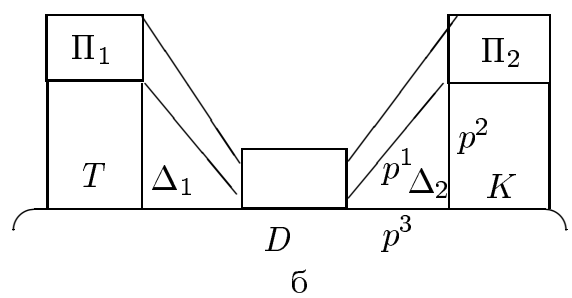

Рис. 10

ЛЕмма 7.8. Если в поддиаграмме $\Delta(S, T, K)$ нет дисков и все ее г-клетки входят в полосу $S$, то некоторая $\left(r_{i}, Q\right)$-клетка полось $S$ имеет общее $Q$-ребро с участком р границы диска $D$, заключенным межсду торцами полос $T$ и $K$ (рис. 10б), а метка $\Sigma \equiv \varphi(p)$ участка р содержит $і$-вхождение.

ДокАЗАТЕльство. По определению диска $\Sigma \equiv q$, или слово $\Sigma h$-специально. Поэтому путь $p$ имеет единственное $Q$-ребро $e$, к которому в $\Delta(S, T, K)$ должна быть приклеена клетка полосы $S$, ибо в клетках полос $T$ и $K$ не может быть $Q$-ребер ни в какой диаграмме над группой $B$.

Пусть $\delta(S, T, K)$ - максимальная поддиаграмма, составленная из всех клеток области $O(S, T, K)$. (Она получается из $\Delta(S, T, K)$ удалением клеток, входящих в полосы $S, T$ и $K$.) Заметим, что среди клеток $r_{i}$-полосы $\left[\Pi_{1}, \ldots, \Pi_{2}\right]$ нет $t$ - и $k$-клеток кроме первой и последней, ибо их наличие привело бы к образованию $\left(t, r_{i}\right)$ - или $\left(k, r_{i}\right)$-кольца вопреки лемме 6.1(5),(6). Поэтому граничная метка диаграммы $\delta(S, T, K)$ - это слово в порождающих группы $B_{2}$, и далее в доказательстве эту поддиаграмму можно рассматривать как диаграмму над $B_{2}$ по лемме 7.3.

Вспоминая определение $t$ - и $k$-полос, приходим к выводу, что к словам, написанньм на $\partial \delta(S, T, K)$, применима лемма 7.1 , в соответствии с которой в границе этой 
поддиаграммы нет других $Q$-ребер, кроме $e^{ \pm 1}$. При удалении этого ребра диаграмма $\delta(S, T, K)$ распадается на две части $\Delta_{1}$ и $\Delta_{2}$ без $Q$-ребер в контурах. В их контурах нет и $r$-ребер, так как все $r$-клетки диаграммы $\Delta(S, T, K)$ входят в полоcу $S$. Значит, по лемме $4.2 \Delta_{1}, \Delta_{2}$ можно считать диаграммами над группой $B_{1}$.

Рассмотрим, например, поддиаграмму $\Delta_{2}$. Ее контур имеет вид $p^{1} p^{2} p^{3}$, где $p^{1}$ входит в край полосы $S, p^{2}$ - в край полосы $K$, а $p^{3}$ - подпуть пути $p^{-1}$. По определению диска $\varphi\left(\left(p^{3}\right)^{-1}\right)$ - положительное слово от $s_{0}, \ldots, s_{M}, h$, а по определению $k$-полосы $\varphi\left(p^{2}\right)-$ слово от $x$.

Слово $U \equiv \varphi\left(p^{1}\right)$ свободно равно произведению вида $G_{i} V_{1} \ldots V_{n}$, где $V_{1}, \ldots, V_{n}$ имеют вид $\left(s_{\beta} x\right)^{ \pm 1}$ или $(h x)^{ \pm 1}$. (Вариант с $K_{i}$ может быть разобран аналогично.) Ввиду минимальности полосы $S$ при приведении слова $U$ к каноническому виду в HNN-расширении $B_{1}$ сокрашения проходных $s$-букв могли бы возникнуть только на стыке $G_{i}$ и $V_{1}$. Но в произведении $G_{i} V_{1}$ встречается тогда ровно одна буква $x^{ \pm 1}$, и оно приведено в $B_{1}$ ввиду положительности слова $G_{i}$.

Таким образом, в приведенной в $B_{1}$ форме слова $U$ началом является слово $G_{i}$. По лемме Бриттона такое же начало должно быть у слова $\varphi\left(\left(p^{3}\right)^{-1}\right)$ (которое отличается от $U$ в $B_{1}$ только правым множителем из $\left.\langle x\rangle\right)$.

После аналогичного рассмотрения поддиаграммы $\Delta_{1}$ получается искомое $i$-вхождение, и лемма доказана.

В заключение параграфа рассмотрим более сложную конфигурацию в некоторой минимальной диаграмме $\Delta$ над группой $B$.

Пусть $D$ - диск, а $S$ - r-полоса, которая последовательно пересекает по одному разу $t$ - и $k$-полосы $S_{1}, \ldots, S_{n}$, определенные циклически занумерованными спицами, выходящими из $D$. Если для любых смежных полос $T=S_{j}, K=S_{j+1}$ (или $\left.K=S_{j}, T=S_{j+1}\right)$ этой системы диаграмма $\Delta(S, T, K)$ не содержит дисков и $n>2 L+1$, то скажем, что полоса $S$ искривляется диском $D$.

Лемма 7.9. В минимальной диаграмме $\Delta$ над группой $B$ нет $r$-полос, искривляемых дисками.

ДокАЗАТЕльство. Допустим, что такая $r$-полоса $S$ имеется, и предположим сначала, что в области $O$, ограниченной средней линией полосы $S$ и спицами с номерами 1 и $n$, есть $r$-клетки. Тогда из них можно составить другую искривляемую $r$-полосу, так как в $O$ не может быть $r$-колец или $(r, t)-,(r, k)$-колец по лемме 6.1 $((2),(5)$ и $(6))$. Поэтому из индуктивных соображений (по числу $r$-клеток в $O$ ) можно считать, что все поддиаграммы $\Delta\left(S, S_{j}, S_{j+1}\right)(j=1, \ldots, n-1)$ удовлетворяют условию леммы 7.8 .

На основании леммы 7.8 в $\Delta$ можно выделить поддиаграмму $\Delta(S)$, составленную из диска $D$ и $(n-1)$-й $\left(r_{i}, Q\right)$-клетки $\pi_{j}$, приклеенных к $D$ вдоль $Q$-ребер в каждом промежутке между торцами полос $S_{1}, \ldots, S_{n}$ (рис. 11a). По определениям (7.3), (4.10) граничной метки диска все клетки $\pi_{1}, \ldots, \pi_{n-1}$ отвечают одному соотношению вида (4.5), причем, находясь в одной $r_{i}$-полосе, они все приклеены единообразно: либо их $r$-ребра направлены “от" $D$, либо у всех - “к" $D$.

Вырежем поддиаграмму $\Delta(S)$ из $\Delta$ и увеличим ее следующим образом. Сначала приклеим $\left(r_{i}, Q\right)$-клетки к оставшимся свободными $4 L-n+1 Q$-ребрам диска $D$ (рис. 11б). Потом с помошью леммы 7.7 приклеим $4 L$ диаграмм меньших рангов. При переходе от одного участка между торцами спиц в $\partial D$ к смежному 


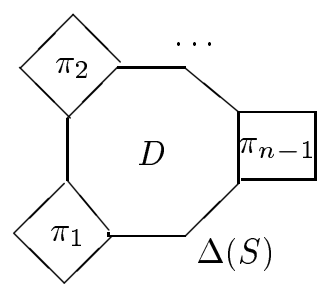

a

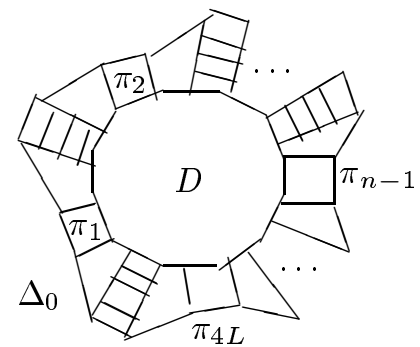

б

Рис. 11

они получаются одна из другой зеркальньм отражением ввиду того, что метки смежных участков взаимно обратны. Поэтому возникающие после таких приклеиваний участки границы с метками, зависящими от $r_{i}, x$, попарно разделены $k$ - и $t$-ребрами диска $D$ и взаимно обратны. На основании соотношений (4.6)-(4.9) эти участки можно заклеить с помощью $4 L$ диаграмм (заштрихованы на рис. 11б) так, что меткой границы, полученной в итоге диаграммы $\Delta_{0}$, будет слово вида $(7.3)$, но с полученным из леммы 7.7 словом $\Sigma_{1}$ вместо $\Sigma$.

Из полученного соотношения вида (7.3) и леммы 7.5 следует, что $\Sigma_{1}=q$ в полугруппе $\Gamma(T)$, т.е. ту же граничную метку, что и диаграмма $\Delta_{0}$, имеет некоторый диск $D_{1}$. Поскольку диаграмма $\Delta_{0}$ получена путем добавления к $\Delta(S)$ некоторой диаграммы (обозначим ее $\Delta^{\prime}$ ), состоящей из $4 L-n+1(r, Q)$-клеток и клеток меньших рангов, диаграмма $\bar{\Delta}(S)$ с той же граничной меткой, что и у $\Delta(S)$, может быть получена путем приклеивания к диску $D_{1}$ зеркальной копии диаграммы $\Delta^{\prime}$. Тип диаграммы $\bar{\Delta}(S)$ меньше, чем у $\Delta(S)$. (Столько же дисков -1 , но менњшее число $(r, Q)$-клеток, ибо $4 L-n+1<n-1$.) Поэтому поддиаграмма $\Delta(S)$ в $\Delta$, а вместе с ней и вся диаграмма $\Delta$, не минимальны.

\section{§8. Отсутствие огибающих $r$-полос в диаграммах над $B_{6}$}

Включение “лишних" соотношений в список определяюших и предписанньй в $\oint 5$ ранг $(\sigma, t)$-клеткам делают возможньм избежать ситуации огибания для $r$ и $\sigma$-полос не только в диаграммах над $B$, но и над группой $B_{6}$.

Определим аналог конструкции $\Delta(S, T, K)$, данной перед леммой 7.8 . Пусть $D$ - диск в минимальной диаграмме $\Delta$ над группой $B_{6}$, соотношения (4.2)-(4.20) которой даны в $\S 4$. Пусть $S-\sigma$ - или $r$-полоса, последовательно пересекающая по одному разу $t$-полосу $T_{1}, k$-полосу $K$ и $t$-полосу $T_{2}$, определенные тремя спицами последовательно выходящими из диска $D$. Обозначим через $\Delta\left(S, T_{1}, T_{2}\right)$ поддиаграмму, составленную из клеток полос $S, T_{1}, T_{2}$ (точнее, как и в $\S 7$, их подполос, выделяемых клетками $\Pi_{1}, \Pi_{2}$, находящимися на пересечении полосы $S$ с $T_{1}$ и $T_{2}$ соответственно) и всех клеток, целиком входящих в область $O\left(S, T_{1}, T_{2}\right)$, ограниченную указанньми двумя $t$-спицами и средней линией полосы $S$.

ЛЕмма 8.1. Если в поддиаграмме $\Delta\left(S, T_{1}, T_{2}\right)$ нет дисков и все ее б-клетки входят в $\sigma$-полосу $S$, то в $\Delta\left(S, T_{1}, T_{2}\right)$ содержится только по одной клетке из полос $T_{1}, T_{2}$, а именно $\Pi_{1}$ и $\Pi_{2}$, которые, следователь- 
но, имеют общие t-ребра грании с диском D. Кроме того, если граничной меткой диска $D$ является левая часть равенства (7.3) с h-специальным словом $\Sigma \equiv h^{-1} X^{\#} q_{j} Y h$, то $q_{j} \equiv q_{1}$, a $X-$ nустое слово, т.е. по лемме 7.5 граничная метка диска $D$ имеет вид (7.4) с $w \equiv Y \in E ;$ диск $D$ не может отвечать соотношению (4.10).

ДокАЗАтЕльство. Поддиаграмма $\Delta\left(S, T_{1}, T_{2}\right)$ является объединением двух поддиаграмм $\Delta_{1}=\Delta\left(S, T_{1}, K\right)$ и $\Delta_{2}=\Delta\left(S, T_{2}, K\right)$ по общим клетками полосы $K$. Как видно из условия леммы, $(\sigma, t)$-клетки могут встречаться в $\Delta_{1}$ только в полоcе $S$. Если бы такие клетки, кроме $\Pi_{1}$, действительно встретились, то неизбежно появление $(\sigma, t)$-колец, ибо в поддиаграмме $\delta\left(S, T_{1}, K\right)$, определенной так же, как в доказательстве леммы 7.8 , граничные $t$-ребра могут лежать только на общей с $S$ границей. Но такие кольца невозможны по лемме 6.1(10).

Заметим далее, что при обходе границы поддиаграммы $\delta\left(S, T_{1}, K\right)$ встречается ровно два $Q$-ребра: одно из них на границе диска $D$, где написано слово $\Sigma$ или одна буква $q$, а другое - на границе $(\sigma, t)$-клетки $\Pi_{1}$, ибо других $(\sigma, t)$ - или $(r, Q)$-клеток в $\Delta_{1}$ нет. Эти два ребра должны быть просто взаимно обратны, поскольку в $\delta\left(S, T_{1}, K\right)$ нет $Q$-клеток. Значит, $q_{j} \equiv q_{1}$. При удалении ребра с этой меткой диаграмма $\delta\left(S, T_{1}, K\right)$ распадается на две диаграммы, на контуре одной из которых написано слово $h^{-1}\left(X^{\#}\right)^{-1} h x^{ \pm l}$, где $l$ - число $(t, x)$-клеток в полосе $T_{1}$ между диском $D$ и клеткой $\Pi_{1}$. (См. соотношения $(4.7),(4.17)$ и (4.18).)

Для доказательства утверждения леммы для клетки $\Pi_{1}$ заметим, что $l=0$. Это следует из леммы 4.2 , если равенство $h^{-1}\left(X^{\#}\right)^{-1} h x^{ \pm l}=1$ выполнено не только в группе $B_{6}$, но и в $B_{2}$. В самом деле, из условия леммы известно, что в вьводе этого соотношения не участвуют $(k, t)$-клетки, а группа, заданная всеми остальньми определяющими соотношениями группы $B_{6}$, допускает очевидную ретракцию на группу $B_{2}$, при которой порождающие $\sigma, d, b_{j}, u_{j}, k_{i}, t_{i}$ при $j=1, \ldots, M$; $i=1, \ldots, L$ отправляются в 1 . Наконец, слово $X^{\#}$, равное $h x^{ \pm l} h^{-1}$ в группе $B_{2}$, должно быть пустым по лемме 4.2 .

Утверждение леммы о П 2 получается из рассмотрения поддиаграммы $\Delta_{2}$.

Лемма 8.2. Для любого слова $w \in E$ равенство слова

$$
\left(h^{-1} q_{1} w h\right) k_{1}\left(h^{-1} q_{1} w h\right)^{-1}
$$

слову $\left(h^{-1} q_{1} d w h\right) k_{1}\left(h^{-1} q_{1} d w h\right)^{-1}$ в группе $B_{6}$ мохсет быть выведено из соотношений этой группь, данных в $\S 4$, без использования $(k, t)$-соотношений (4.10) и $(\sigma, t)$-соотношений (4.17), (4.18).

ДокАЗАТЕЛЬСтво. В силу соотношений (4.16) и (4.13) $d w=w w_{b} d$, а $d\left(h k_{1} h^{-1}\right) d^{-1}=h k_{1} h^{-1}$ ввиду (4.15). Поэтому задача сводится к сравнению слов $w_{b} h k_{1} h^{-1} w_{b}^{-1}$ и $h k_{1} h^{-1}$, т.е. к доказательству перестановочности слов $w_{b}$ и $h k_{1} h^{-1}$. Но, действительно, из соотношений (4.14), (4.12) и (4.11) получаем: $\left(h k_{1} h^{-1}\right)^{-1} w_{b}\left(h k_{1} h^{-1}\right)=w_{b} w_{u}$, причем $w_{u}=1$, так как $w_{u} \in E_{u}$.

Лемма 8.3. Пусть $D-$-иск в некоторой диаграмме $\Delta$ над группой $B_{6}$, граница которого имеет общие $t$-ребра с каждой из $n(\sigma, t)$-клеток $\Pi_{1}, \ldots, \Pi_{n}$, причем эти клетки единообразно приклеены $\kappa D$, т.е. либо все их б-ребра направлень от $D$, либо все их $\sigma$-ребра направлены $\kappa D$. Тогда если граничная метка диска $D$ имеет вид (7.4), а $n>L$, то диаграмма $\Delta$ не минимальна. 
ДокАЗАТЕЛЬСТво. Достаточно показать, что не является минимальной поддиаграмма $\Delta_{1}$, составленная из диска $D$ и клеток $\Pi_{1}, \ldots, \Pi_{n}$ (рис. $\left.12 \mathrm{a}\right)$.

Рассматривая диаграмму $\Delta_{1}$ отдельно, проведем следующие дополнительные построения. Сначала приклеим $2 L-n$ новых $(\sigma, t)$-клеток к каждому из оставшихся свободными $2 L-n t$-ребер в $\partial D$ (рис. $12 \sigma)$, причем так, чтобы $\sigma$-ребра всех $2 L(\sigma, t)$-клеток в полученной таким образом диаграмме $\Delta_{2}$ были направлены к $D$ (случай $\mathrm{A})$, либо все - от $D$ (случай Б).

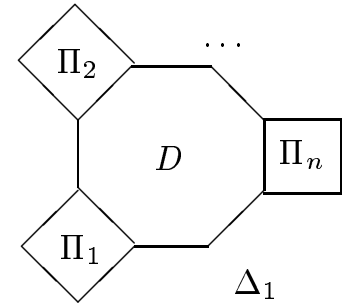

a

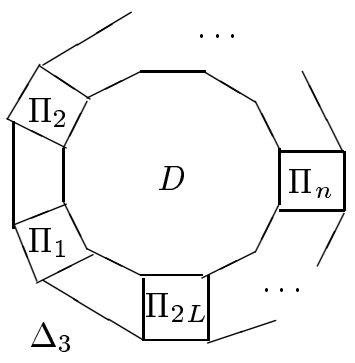

б

Рис. 12

Поскольку между $t$-ребрами границы диска $D$ расположены подпути с метками вида $U_{j}^{ \pm 1}$, где $U_{j} \equiv\left(h_{1}^{-1} q_{1} w h\right) k_{j}^{ \pm 1}\left(h^{-1} q_{1} w h\right)^{-1}, w \in E$, граничной меткой диаграммы $\Delta_{2}$ будет слово

$$
\prod_{j=1}^{L} V_{j} t_{j} V_{j}^{\prime} t_{j}^{-1}
$$

где в случае А

$$
\begin{aligned}
& V_{1}^{\prime} \equiv\left(h^{-1} q_{1} \sigma d^{-1} q_{1}^{-1} h\right) U_{1}\left(h^{-1} q_{1} \sigma d^{-1} q_{1}^{-1} h\right)^{-1}, \\
& V_{1} \equiv\left(h^{-1} q_{1} \sigma q_{1}^{-1} h\right) U_{1}^{-1}\left(h^{-1} q_{1} \sigma q_{1}^{-1} h\right)^{-1}
\end{aligned}
$$

и

$$
V_{j}^{-1} \equiv V_{j}^{\prime} \equiv\left(h^{-1} q_{1} \sigma q_{1}^{-1} h\right) U_{j}\left(h^{-1} q_{1} \sigma q_{1}^{-1} h\right)^{-1}
$$

при $j>1$, а в случае Б

$$
\begin{aligned}
& V_{1}^{\prime} \equiv\left(h^{-1} q_{1} d \sigma^{-1} q_{1}^{-1} h\right) U_{1}\left(h^{-1} q_{1} d \sigma^{-1} q_{1}^{-1} h\right)^{-1}, \\
& V_{1} \equiv\left(h^{-1} q_{1} \sigma^{-1} q_{1}^{-1} h\right) U_{1}^{-1}\left(h_{-1} q_{1} \sigma^{-1} q_{1}^{-1} h\right)^{-1}
\end{aligned}
$$

и

$$
V_{j}^{-1} \equiv V_{j}^{\prime} \equiv\left(h^{-1} q_{1} \sigma^{-1} q_{1}^{-1} h\right) U_{j}\left(h^{-1} q_{1} \sigma^{-1} q_{1}^{-1} h\right)^{-1}
$$

при $j>1$. (См. соотношения (4.17) и (4.18).)

В случае А в слове $V_{1}^{\prime}$ после сокращений буква $d$ может быть также вычеркнута по лемме 8.2 без использования соотношений вида $(4.10),(4.17)$ и (4.18). Если потом применить соотношения (4.19) и (4.20), то можно вычеркнуть и вхождения буквы $\sigma$, что приводит к слову $U_{1}$. В случае Б слово $V_{1}^{\prime}$ также равно слову $U_{1}$ в силу 
соотношений группы $B_{6}$, но без использования соотношений (4.10), (4.17), (4.18). (В этом случае сначала избавляемся от $\sigma$, а потом от $d$.) Аналогичное равенство каждого из слов $V_{j}^{\prime}$ слову $U_{j}$ при $j>1$ объясняется проще, так как не требуется ссылка на лемму 8.2.

Проведенные выкладки означают, что с помощью клеток, ранги которых меньше, чем ранги $(k, t)$ - и $(\sigma, t)$-клеток, диаграмма $\Delta_{2}$ может быть достроена до диаграммы $\Delta_{3}$ с такой же граничной меткой, как у диска $D$ (рис. 12б). Поскольку диаграмма $\Delta_{3}$ получена из диаграммы $\Delta_{1}$ с помощью добавления некоторой диаграммы (обозначим ее $\Delta^{\prime}$ ), состояшей из $2 L-n(\sigma, t)$-клеток и клеток меншего ранга, диаграмма $\bar{\Delta}$ с той же граничной меткой, что и у $\Delta_{1}$, может быть получена посредством приклеивания к $D$ зеркальной копии диаграммы $\Delta^{\prime}$. Но тип диаграммы $\bar{\Delta}$ меньше типа диаграммы $\Delta_{1},-$ у нее меншше $(\sigma, t)$-клеток, ибо $2 L-n<n$. Поэтому диаграмма $\Delta_{1}$ не минимальна.

Пусть $D$ - диск в диаграмме $\Delta$ над группой $B_{6}$, а $S-r$ - или $\sigma$-полоса, последовательно пересекающая по одному разу смежные $t$ - и $k$-полосы $T_{1}, K_{1}, T_{2}$, $K_{2}, \ldots, T_{n}$, определенные последовательньми смежными спицами диска $D$. Если при этом поддиаграммы $\Delta\left(S, T_{j}, T_{j+1}\right)$ (определенные в начале параграфа) не содержат дисков, а $n>L+1$, то скажем, что полоса $S$ огибает диск $D$.

ЛЕмма 8.4. В минимальной диаграмме $\Delta$ над группой $B_{6}$ нет $r$ - или $\sigma$-полос, огибающих диски.

ДокАЗАтЕльство. Доказывая лемму “от противного", можно считать, что все $r$ - или $\sigma$-клетки поддиаграмм $\Delta_{j}=\Delta\left(S, T_{j}, T_{j+1}\right)$ входят в полосу $S$. (Это достигается путем выбора "ближайшей" к $D$ огибающей полосы, как и в начале доказательства леммы 7.9 с добавлением ссылок на пункты (1) и (9), (10) леммы 6.1 при исключении $\sigma$-клеток.)

Допустим сначала, что $S$ состоит из $r$-клеток. Тогда на границе каждой из поддиаграмм $\Delta_{j}$ нет $\sigma$-ребер. Поэтому по лемме 6.1(1) в этих диаграммах нет $\sigma$-клеток, т.е. нет $d$-терминальных клеток. Поскольку в $\partial \Delta_{j}$ нет $d$-ребер, в $\Delta_{j}$ нет $d$-клеток по лемме 6.2(18). Аналогично исключаются $b$-клетки по лемме 6.1(19), а значит, и $(u, u)$-клетки. Поэтому $\Delta_{j}$ - диаграмма над группой $B$, а полоса $S$ оказывается искривляемой диском $D$ в некоторой поддиаграмме диаграммы $\Delta$, определенной над $B$, так как $2 n-1>2 L+1$. Но это противоречит лемме 7.9.

Пусть теперь $S-\sigma$-полоса. Применяя в этом случае лемму 8.1 к каждой из поддиаграмм $\Delta_{j}$, получаем $n(\sigma, t)$-клеток $\Pi_{1}, \ldots, \Pi_{n}$, единообразно приклеенных к диску $D$, ибо они входят в одну полосу $S$. Кроме того, граничная метка диска $D$ должна иметь вид (7.4). В таком случае утверждение леммы следует из леммы 8.3.

\section{§9. Диски и спицы в диаграммах над группой $B_{6}$}

Этот параграф̆ посвящен описанию трюка, с помощью которого можно избавляться от пары дисков, связанных несколькими спицами не только в диаграммах над группой $B$ (типа Новикова-Буна), но и над группой $B_{6}$ (типа Андера).

Лемма 9.1. Пусть для слова $w \in$ Е составлено произведение (7.4), в котором как в ииклическом слове выбрано подслово $V_{0}$, полученное вычеркиванием одного из дополнительных подслов вида $q_{1}^{-1} h t_{j} h^{-1} q_{1} w h k_{j} h^{-1}$, где $j \neq 1$ или 
$h k_{j} h^{-1} w^{-1} q_{1}^{-1} h t_{j}^{-1} h^{-1} q_{1}$, где $j \neq 1$, или $q_{1}^{-1} h t_{j}^{-1} h^{-1} q_{1} w h k_{j+1}^{-1} h^{-1}$, где $j \neq 1$ ( а индексы рассматриваются по модулю L), или $h k_{j}^{-1} h^{-1} w^{-1} q_{1}^{-1} h t_{j} h^{-1} q_{1}$, где $j \neq 1$. (То есть, индекс $t$-буквы всегда отличен от 1.) Тогда равенство $V_{0} \sigma^{-1} V_{0}^{-1} \sigma=1$ в группе $B_{6}$ можно вивести из определяющих соотношений этой группь, данных в $\$ 4$, без использования $(k, t)$-соотношений (4.10).

ДокАЗАТЕЛЬство. Буква $\sigma$ перестановочна со словом $w$ ввиду соотношений (4.20), а с произведениями вида $q_{1}^{-1} h t_{j} h^{-1} q_{1}$ при $j \neq 1$ и с произведениями $h k_{j} h^{-1}$ в силу (4.18) и (4.19). Поэтому для доказательства леммы достаточно слово $V_{0}$ заменить его подсловом $V \equiv\left(q_{1}^{-1} h t_{1} h^{-1} q_{1}\right) w h k_{1} h^{-1} w^{-1}\left(q_{1}^{-1} h t_{1}^{-1} h^{-1} q_{1}\right)$.

Ввиду соотношений (4.17), (4.20) и (4.19)

$$
\sigma^{-1} V \sigma=\left(q_{1}^{-1} h t_{1} h^{-1} q_{1} d\right) w\left(h k_{1} h^{-1}\right) w^{-1}\left(d^{-1} q_{1}^{-1} h t_{1}^{-1} h^{-1} q_{1}\right) .
$$

K подслову правой части равенства (9.1), заключенному между двумя вхождениями буквы $t_{1}$, можно применить лемму 8.2. С ее помощью можно вычеркнуть оба вхождения буквы $d$, после чего правая часть преврашается в слово $V$, что и требовалось.

Рассмотрим теперь вспомогательную конфигурацию $\Delta(S, T, K)$, определяемую некоторьм диском диаграммы $\Delta$, выходящими из него смежными $t$ - и $k$-спицами и полосой $S$, как она была определена перед леммой 7.8 , с той лишь разницей, что $S$ - $\sigma$-полоса (а не $r$-полоса), а $\Delta$ - диаграмма над группой $B_{6}$ (а не $B$ ). Обозначение $\delta(S, T, K)$ будем употреблять, как и в доказательстве леммы 7.8 , для поддиаграммы, заключенной внутри области, ограниченной двумя спицами и средней линией полосы $S$. Если $\Pi_{1}$ и $\Pi_{2}$ - клетки, обшие для полос $T, S$ и $K, S$ соответственно, то подполосу полосы $S$ вида $\left[\Pi_{1}, \pi_{1}, \ldots, \pi_{n}, \Pi_{2}\right]$ обозначим $S_{1}$.

ЛЕмма 9.2. Если в приведенных обозначениях поддиаграмма $\Delta(S, T, K)$ минимальна и не содержит дисков, все ее б-клетки входят в полосу $S_{1}$, а полоса $T$ является $t$-полосой для $t \neq t_{1}$, то метки обоих краев полосы $S_{1}$ одинаковы и имеют вид $W \equiv q_{1}^{-1} h t^{ \pm 1} h^{-1} q_{1} w h k^{ \pm 1} h^{-1}$, әде $t \in\left\{t_{2}, \ldots, t_{L}\right\}$, $k \in\left\{k_{1}, \ldots, k_{L}\right\}, a w \in E$.

ДокАЗАТЕЛЬСТво. Граничные $t$ - или $k$-ребра поддиаграммы $\delta(S, T, K)$ могли бы входить только в границы клеток $\pi_{1}, \ldots, \pi_{n}$ полосы $S_{1}$. Но тогда неизбежно появление $(\sigma, t)$ - или $(\sigma, k)$-колец вопреки утверждениям $(10)$ и $(9)$ леммы 6 . Поэтому клетки $\pi_{1}, \ldots, \pi_{n}$-это $(\sigma, a)$-клетки. Учитывая вставки с метками $\left(h^{-1} q_{1}\right)^{ \pm 1}$ между $t$ - и $\sigma$-ребрами в клетке $\Pi_{1}$ и аналогичные вставки $h^{ \pm 1}$ в $\Pi_{2}$, получаем, что $W \equiv q_{1}^{-1} h t^{ \pm 1} h^{-1} q_{1} w h k^{ \pm 1} h^{-1}$, где $w$ - слово от $a_{1}, \ldots, a_{m}$. При этом метки краев полосы $S_{1}$ совпадают ввиду того, что все ее клетки отвечают соотношениям коммутации (4.18)-(4.20).

Поскольку в $\partial \delta\left(S_{1}, T, K\right)$ нет $\sigma$-, $d-, b$-, $t$ - и $k$-ребер, то на основании леммы 6.1 (пункты (1), (18), (19), (7) и (8)) последовательно заключаем, что в $\delta(S, T, K)$ нет $\sigma-, d-, b-, t$ - и $k$-клеток. Следовательно нет и $(u, u)$-клеток, т.е. $\delta(S, T, K)$ - это диаграмма над группой $B_{2}$. Из нее получается равенство вида $L^{\prime} h^{-1} q_{1} w h L^{\prime \prime}=\Sigma$, где слова $L^{\prime}, L^{\prime \prime}$ зависят от $x, r_{i} ; i \in I$, а $\Sigma=q$ в полугрупше $\Gamma(T)$ по определению граничной метки диска $D$. По лемме 4.5 для слова $\Sigma$ выполнено соотношение (7.3), откуда по лемме $7.4 q=L_{1} \Sigma L_{2}=L_{1} L^{\prime} h^{-1} q_{1} w h L^{\prime \prime} L_{2}$ в группе $B_{2}$ для некоторых 
слов $L_{1}, L_{2}$ от $x, r_{i} ; i \in I$. Отсюда по лемме $4.4 h q_{1} w h=q$ в полугруппе $\Gamma(T)$ и $w$ - положительное слово. Наконец, по лемме 4.1 получается, что $w \in E$.

Лемма 9.3. Пусть диаграмма $\Delta$ над группой $B_{6}$ содержит два диска $D_{1} u$ $D_{2}$ с общими смежными $t$ - и $k$-спицами, причем $t \neq t_{1}$, а в области $O$ между әтими спицами нет других дисков и нет других спиц. Тогда диаграмма $\Delta$ не является минимальной: при сохранении ее граничной метки число дисков можно уменьшить на два.

ДокАЗАТЕльство. Для доказательства можно считать, что в диаграмме нет иных клеток, кроме дисков $D_{1}, D_{2}$, клеток полос $T$ и $K$, через которые проходят общие спицы дисков, и клеток, целиком содержащихся в области $O$. Можно считать также, что поддиаграмма $\bar{\Delta}$, составленная из тех же клеток, но без дисков (заштрихована на рис.13a), является минимальной, так как при ее приведении к минимальному виду единственная пара граничных $t$-ребер и пара $k$-ребер по-прежнему останутся торцами максимальной $t$ - и $k$-полосы.

Выделим далее в $\bar{\Delta}$ максимальные $\sigma$-полосы $S_{1}, \ldots, S_{n}$. (Возможно, $n=0$ ) Поскольку по лемме $6.1(9),(10)$ в $\bar{\Delta}$ нет $(\sigma, t)$ - и $(\sigma, k)$-колец, а в $\partial D_{1}, \partial D_{2}$ нет $\sigma$-ребер, первая и последняя клетка каждой полосы $S_{1}, \ldots, S_{n}$ входят в полосы $T$ и $K$ соответственно. Это позволяет считать, что $\sigma$-полосы занумерованы “от $D_{1}$ к $D_{2} "$.

Утверждение леммы будет доказываться индукцией по числу $n$ максимальных $\sigma$-полос в $\bar{\Delta}$. Заметим, что $2 n-$ это число $\sigma$-ребер в $\partial \Delta$.

Если $n=0$, то в гранище диаграммы $\Delta$ нет $\sigma$-ребер. Нет также и $d$-ребер, ибо $t \neq t_{1}$. Очевидно также, что в $\partial \Delta$ нет $b$ - и $u$-ребер. Поэтому по утверждениям (1), (18) и (19) леммы 6.1 в диаграмме $\bar{\Delta}$ нет $\sigma-, d-, b$ - и $u$-клеток, т.е. $\bar{\Delta}$ - это диаграмма над группой $B$. В таком случае утверждение леммы следует из леммы 7.6.

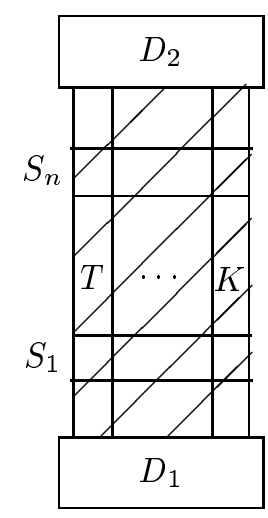

a

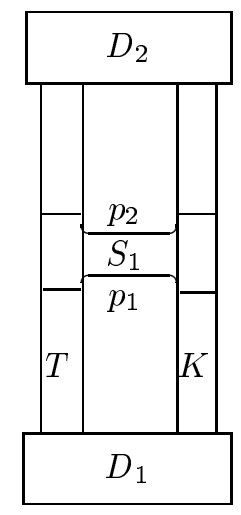

б

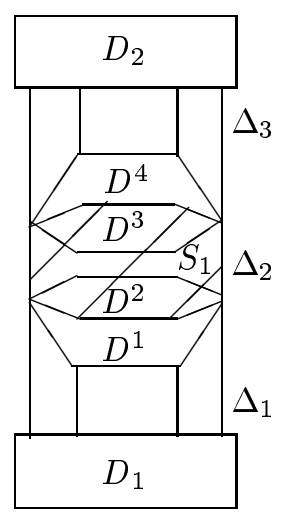

B

Рис. 13

При $n \geqslant 1 \sigma$-полоса $S_{1}$ вместе с полосами $T, K$ и диском $D_{1}$ образуют конфигурацию, к которой применима лемма 9.2. Поэтому края $p_{1}, p_{2}$ полосы $S_{1}$ (рис. $13 б$ ) имеют одинаковую метку $W \equiv q_{1}^{-1} h t^{ \pm 1} h^{-1} q_{1} w h k^{ \pm 1} h^{-1}$ для некоторого слова $w \in E$. 
По лемме 4.1 можно построить вспомогательную диаграмму-диск из одной клетки $D^{1}$ с граничной меткой (7.4), в которой слово $W$ будет подсловом. Обозначим через $\bar{W}$ циклически дополнительное к $W$ подслово в слове (7.4). $\mathrm{K}$ диску $D^{1}$ приклеим его зеркальную копию $D^{2}$ по подпутям границ с одинаковой меткой $\bar{W}$. Получим диаграмму $D^{12}$ с граничной меткой побуквенно равной слову $W W^{-1}$.

Равенство $\varphi\left(p_{1}\right) \equiv W$ позволяет провести следующую операцию над диаграммой $\Delta$ : разрезать ее вдоль пути $p_{1}$ и вклеить в образовавшуюся дыру диаграмму $D^{12}$ (рис. 13в). Аналогично, вдоль разреза по другому краю $p_{2}$ полосы $S_{1}$ можно вклеить диаграмму $D^{34}$, составленную из взаимно зеркальных копий двух дисков с граничной меткой (7.4).

Полученную диаграмму $\Delta^{\prime}$ можно считать также составленной из следующих трех частей $\Delta_{1}, \Delta_{2}$ и $\Delta_{3}$ : средняя состоит из дисков $D^{2}, D^{3}$ и полосы $S_{1}$ между ними. В две оставшиеся после вырезания из $\Delta^{\prime}$ компоненты $\Delta_{1}$ и $\Delta_{3}$ входят соответственно $D_{1}, D^{1}$ и $D^{4}, D_{2}$ вместе со всеми прочими клетками диаграммы $\Delta^{\prime}$.

Из определения диаграммы $\Delta_{2}$ видно, что ее граничная метка имеет вид $\bar{W} \sigma^{ \pm 1} \bar{W}^{-1} \sigma^{\mp 1}$. Здесь слово $\bar{W}$ удовлетворяет условиям леммы 9.1 (с $\left.V_{0} \equiv \bar{W}\right)$, так как $t \neq t_{1}$. Поэтому поддиаграмму $\Delta_{2}$ можно заменить в $\Delta^{\prime}$ некоторой диаграммой без дисков.

В границе диаграммы $\Delta_{1}$ уже нет $\sigma$-ребер. Ввиду разобранного выше случая, когда $n=0$, эту поддиаграмму в $\Delta^{\prime}$ тоже можно заменить на диаграмму без дисков. Наконец, к поддиаграмме $\Delta_{3}$ можно применить индуктивное предположение, так как ее граница содержит $2(n-1) \sigma$-ребер.

Проведенная хирургия позволила получить диаграмму с той же граничной меткой, какая имеется у $\Delta$, но без дисков.

Обозначим через $\Gamma_{t}(\Delta)$ граф, построенньй для произвольной диаграммы $\Delta$ над группой $B_{6}$ следующим образом. Его внутренними вершинами считаются все точки $o_{D}$ внутри дисков, а внешней - вершина $o_{\Delta}$; ребрами же являются все $t$-спицы; однако, если два диска оказьваются соединенньпи двумя $t_{1}$-спицами, то из них включается в $\Gamma_{t}(\Delta)$ только одна (любая).

ЛЕмма 9.4. Если диаграмма $\Delta$ минимальна и содержит диски, то $\Gamma_{t}(\Delta)$ является $(2 L-1)$-графом.

ДокАЗАТЕЛьство. В графе $\Gamma_{1}$ нет одноугольников, так как между $t$-спицами из каждого диска диаграммы $\Delta$ выходят $k$-спицы, и наоборот.

Допустим, некоторые две спищы графа $\Gamma_{t}$, исходящие из диска $D_{1}$ и входящие в диск $D_{2}$, образуют двухугольник. Очевидно, что и все промежуточные $k$-спицы, выходящие из $D_{1}$, кончаются в $D_{2}$. Поэтому среди них найдутся смежные $t$ - и $k$-спицы с $t \neq t_{1}$. Но это противоречит лемме 9.3 .

Поскольку обшее число $t$-спиц каждого диска равно $2 L>7$, лемма доказана.

ЛЕмма 9.5. В минимальной диаграмме над группой $B_{6}$ нет $r$-колец.

ДокАЗАтЕЛьство. Допустим, что $S-r$-кольцо, и рассмотрим максимальную поддиаграмму $\Delta$, лежашую в области, ограниченной его медианой. По лемме $6.1(2)$ в диаграмме $\Delta$ есть диски. Поэтому по леммам 9.4 и 3.2 (а также лемме $6.1(5),(6))$ в графе $\Gamma_{t}(\Delta)$ найдется диск, огибаемый полосой $S$. (Напомним, что $2 L-1-3>$ $L+1$.) Но это противоречит лемме 8.4. 


\section{§10. Сравнение длин элементов некоторых подгрупп}

Прежде, чем провести сравнение длин, необходимое для доказательства теоремы 3, в настояшем параграфе проводятся сравнения длин элементов в некоторых промежуточных подгруппах. Но сначала докажем, что $R_{u} \cong R$ - подгруппа в $B_{6}$.

ЛЕмма 10.1. Естественный гомоморфизм $R_{u} \rightarrow B_{6}$ инбективен.

ДокАЗАТЕльство. Рассмотрим минимальную диаграмму $\Delta$ над группой $B_{6}$ для равенства некоторого $u$-слова $U$ единице. По определению в $\partial \Delta$ нет $t$-ребер, а поэтому в диаграмме $\Delta$ не может быть дисков по леммам 9.4 и 3.4 , ибо $2 L-1>6$. Из утверждений $(1),(18)$ и (19) леммы 6.1 последовательно заключаем, что в $\Delta$ нет $\sigma-, d$ - и $b$-клеток, т.е. слово $U$ равно 1 в группе $B * R_{u}$, а значит, и в группе $R_{u}$.

Итак, можно считать, что $R_{u}$ - это подгруппа в группе $B_{6}$. Введем далее цепь $R_{u} \leqslant A_{1} \leqslant A_{2} \leqslant A_{3} \leqslant A_{4} \leqslant B_{6}$ со следуюшими промежуточными подгруппами.

Положим $A_{1}=g p\left\{R_{u}, b_{1}, \ldots, b_{m}\right\}, A_{2}=g p\left\{A_{1}, k_{1}\right\}, A_{3}=g p\left\{A_{2}, a_{1}, \ldots, a_{m}\right\}$ и $A_{4}=g p\left\{A_{3}, x, d, \sigma, r_{i} ; i \in I\right\}$. Системы порождающих этих подгрупп выделяются естественно: $\left\{u_{1}, \ldots, u_{m}, b_{1}, \ldots, b_{m}\right\}$ - для подгрупшы $A_{1}$ и т. д.

Ниже запись $|V|_{u}$ означает число вхождений букв $u_{1}^{ \pm 1}, \ldots, u_{m}^{ \pm 1}$ в слово $V$, т.е. длину слова после вычеркивания всех других букв.

Лемма 10.2. Пусть и-слово $U$ не равно в $B_{6}$ никакому и-слову меньшей длинь, а $V$ - слово в порожсдающих подгруппь $A_{1}$, равное слову $U$ в групnе $B_{6}$. Тогда $|V|_{u} \geqslant|U|_{u}(=\|U\|)$.

ДокАЗАТЕльство. Пусть $\Delta$ - минимальная диаграмма равенства слов $U$ и $V$. Как и в доказательстве леммы 10.1 , заключаем, что в $\Delta$ нет дисков, $\sigma$ - и $d$-клеток. Значит, $\Delta$ - диаграмма над группой $B_{4}$, и утверждение следует из леммы 4.6 .

Далее через $|W|_{u, b}$ обозначим число вхождений букв $u_{1}^{ \pm 1}, \ldots, u_{m}^{ \pm 1}, b_{1}^{ \pm 1}, \ldots, b_{m}^{ \pm 1}$ в слово $W$.

Лемма 10.3. Пусть $W$ - слово в порожсдающих подгруппь $A_{2}$, представляющее әлемент из подгруппы $A_{1}$, a $V$ - такое несократимое слово в порождающих подгруппь $A_{1}$, для которого диаграмма $\Delta$ равенства $W=V \boldsymbol{в}$ группе $B_{6}$ имеет минимальный тип среди всех диаграмм равенств $W=V^{\prime}$ для слов $V^{\prime}$ в порохсдающих группь $A_{1}$. Тогда $|W|_{u, b} \geqslant|V|_{u}$.

ДокАзАтЕльство. Как и в леммах $10.1,10.2$, диаграмма $\Delta$ не содержит дисков, а затем из нее исключаются $\sigma$ - и $d$-клетки с помощью утверждений $(1)$ и $(18)$ леммы 6.1.

Введем обозначение $p_{V} p_{W}^{-1}$ для контура диаграммы $\Delta$, где $\varphi\left(p_{V}\right) \equiv V, \varphi\left(p_{W}\right) \equiv W$.

Заметим сначала, что путь $p$ простой, в частности, если $e-$ ребро пути $p_{V}$, то ребро $e^{-1}$ не входит в путь $p_{V}$. Действительно, в противном случае от $\Delta$ можно было бы отрезать поддиаграмму, ограниченную петлей пути $p_{V}$, удаляя из $V$ единичное в группе $B_{5}$ подслово и понижая тип диаграммы $\Delta$. Но это противоречило бы условию леммы, наложенному на $V$.

Никакая $(u, b)$ - или $(u, u)$-клетка диаграммы $\Delta$ не может иметь общих граничных ребер с путем $p_{V}$, так как иначе можно было бы вырезать такую клетку из $\Delta$ и заменить соответственно путь $p_{V}$ на путь $p_{V^{\prime}}$ с понижением типа диаграммы вопреки условию леммы, наложенному на слово $V$. 
Поэтому для каждого $u$-ребра $e$ пути $p_{V}$ имеется лишь две возможности:

(1) ребро $e^{-1}$ входит в путь $p_{W}^{-1}$;

(2) ребро е лежит на границе некоторой $\left(u, b, k_{1}\right)$-клетки П. (См. (4.14).)

Во втором случае выберем в $\partial П ~ b$-ребро, которое не смежно с $u$-ребром $e$, и продолжим максимально $b$-полосу $S=\left[\Pi, \Pi_{1}, \ldots, \Pi_{n}\right]$ за это ребро (рис. $\left.14 \mathrm{a}\right)$. По лемме 6.1(19) эта полоса не может оказаться кольцом.

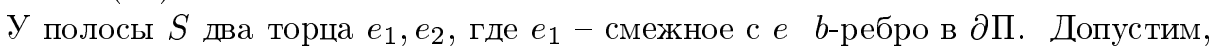
что торец $e_{2}$ полосы $S$ оказался на пути $p_{V}$ (рис. 14б). В этом случае полоса $S$ вместе с путем $p_{V}$ ограничивают некоторую максимальную поддиаграмму $\delta(S)$, в контуре которой есть $k_{1}$-ребро $f$ из $K=\left[\Pi, \pi_{1}, \ldots\right]$, начатую с П и максимально продолженную за $f$. Полоса $K$ должна еще раз пересечь полосу $S$, так как в пути $p_{V}$ нет $k_{1}$-ребер. Но в таком случае из полос $S$ и $K$ можно составить $(b, k)$-кольцо вопреки лемме $6.1(13)$.

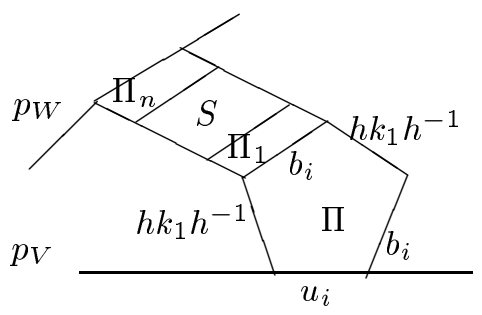

a

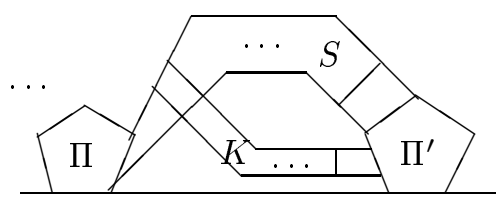

B

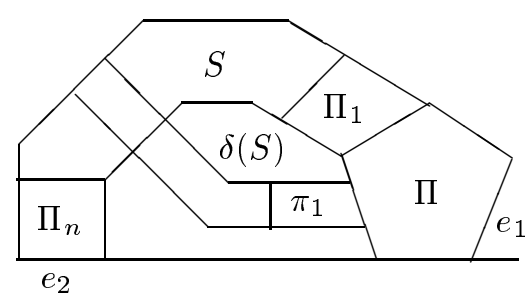

б

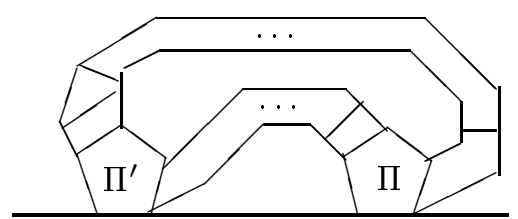

$\Gamma$

Рис. 14

Таким образом, торец $e_{2}$ полосы $S$ обязательно лежит на пути $p_{W}$.

Заметим еще, что клетка П не может входить в $b$-полосу $S^{\prime}$, начатую с другой $\left(u, b, k_{1}\right)$-клетки $\Pi^{\prime}$, имеющей обшее граничное $u$-ребро $e^{\prime} \neq e$ с путем $p_{V}$, так как это опять с неизбежностью приводит к $\left(b, k_{1}\right)$-кольцу или к $b$-кольцу (см. варианты на рис. 14в,г) вопреки лемме 6.1(13). Поэтому построенные таким образом $b$-полосы $S$ и $S^{\prime}$ для разных $u$-ребер $е$ и $e^{\prime}$ пути $p_{V}$ имеют разные торцы на пути $p_{W}$.

Итак, разным $u$-ребрам пути $p_{V}$ в случае $(1)$ сопоставлены разные $u$-ребра пути $p_{W}$, a в случае $(2)$ - разные $b$-ребра пути $p_{W}$. Отсюда следует утверждение леммы.

Лемма 10.4. Пусть $X$ - слово в порождающих подгруппь $A_{3}$, представляющее некоторый элемент из $A_{2}$, a $W$ - такое несократимое слово в порожддающих подгруппь $A_{2}$, для которого диаграмма $\Delta$ равенства $X=W$ в группе $B_{6}$ имеет минимальный тип среди всех диаграмм равенств $X=W^{\prime}$ для слов $W^{\prime}$ в порождающих группь $A_{2}$. Тогда $|X|_{u, b} \geqslant|W|_{u, b}$. 
ДокАЗАТЕльство. Как и в леммах 10.1-10.3, диаграмма $\Delta$ не содержит дисков, а также $\sigma$ - и $d$-клеток. По лемме 6.1(4) нет в ней и $Q$-клеток, т.е. терминальных клеток для $a$-полос. Ее границу обозначим $p_{W} p_{X}^{-1}$, где $\varphi\left(p_{W}\right) \equiv W$, $\varphi\left(p_{X}\right) \equiv X$. Как и в лемме 10.3 , путь $p_{W}$ является простым, но теперь никакое его $u$-ребро $e$ не может принадлежать границе не только $(u, b)$ - или $(u, u)$-клетки, но и $\left(u, b, k_{1}\right)$-клетки. Поэтому ребро $e$ должно также входить и в путь $p_{X}$.

По той же причине, если ребро $е$ пути $p_{W}$ является $b$-ребром, то для него имеется только две возможности:

(1) ребро $е$ входит в путь $p_{X}$;

(2) е лежит на границе некоторой $(b, a)$-клетки, т.е. является торцом некоторой максимальной $b$-полосы $S$.

Если второй торец полосы $S$ также входит в путь $p_{W}$, то можно определить максимальную поддиаграмму $\delta(S)$, ограниченную путем $p_{W}$ и медианой полосы $S$. Наличие в $S \quad(b, a)$-клетки позволяет построить некоторую максимальную $a$-полосу $T$, которая должна дважды пересекаться с полосой $S$, образуя $(b, a)$-кольцо, поскольку в пути $p_{W}$ нет $a$-ребер. Но это противоречит лемме $6.1(12)$. Следовательно, в случае (2) второй торец полосы $S$ является $b$-ребром пути $p_{X}$.

Таким образом, разным $b$-ребрам пути $p_{W}$ сопоставлены разные $b$-ребра пути $p_{X}$. Поскольку для $u$-ребер это было сделано еше раньше, лемма доказана.

Через $|Y|_{u, b, a}$ обозначается далее число вхождений букв $u_{1}^{ \pm 1}, \ldots, u_{m}^{ \pm 1}$, $b_{1}^{ \pm 1}, \ldots, b_{m}^{ \pm 1}, a_{1}^{ \pm 1}, \ldots, a_{m}^{ \pm 1}$ в слово $Y$. (Аналогично определяется величина $|Y|_{u, b, s}$, которая не меньше $|Y|_{u, b, a}$, так как $a$-буквы являются $s$-буквами.)

Лемма 10.5. Пусть $Y$ - слово в порождающих подгруппьи $A_{4}$, представляющее некоторый әлемент из $A_{3}$, a $X$ - такое несократимое слово в порождающ, их подгруппь $A_{3}$, для которого диаграмма $\Delta$ равенства $Y=X$ в группе $B_{6}$ имеет минимальный тип среди всех диаграмм равенств $Y=X^{\prime}$ для слов $X^{\prime}$ в порожсдающих подгруппь $A_{3}$. Тогда $|Y|_{u, b, a} \geqslant|X|_{u, b}$.

ДокАЗАТЕЛЬСтво. Как и в леммах 10.1-10.4, диаграмма $\Delta$ не содержит дисков, а по лемме 6.1 (7) в ней нет $t$-клеток, ибо в границе $\partial \Delta$ нет $t$-ребер. Из утверждения (4) леммы 6.1 следует также, что в $\Delta$ нет $Q$-клеток, ибо нет $Q$-терминальных клеток, а $\partial \Delta$ не имеет $Q$-ребер.

Контур диаграммы $\Delta$ имеет вид $p_{X} p_{Y}^{-1}$, где $\varphi\left(p_{X}\right) \equiv X, \varphi\left(p_{Y}\right) \equiv Y$. Как и в лемме 10.4, путь $p_{X}$ должен быть простым, а любое его $u$-ребро должно содержаться в пути $p_{Y}$.

В силу условия на слово $X$ никакое $b$-ребро $e$ пути $p_{X}$ не входит в границу $(b, u)$-, $\left(b, k_{1}\right)$ - или $(b, a)$-клетки, так как такую клетку можно было бы вырезать из $\Delta \mathrm{c}$ переходом к диаграмме равенства $Y=X^{\prime}$ меншшего типа. Поэтому для $e$ имеется две возможности:

(1) $b$-ребро $e$ пути $p_{X}$ входит также в путь $p_{Y}$;

(2) $b$-ребро е лежит на границе некоторой $(d, a, b)$-клетки П. (См. (4.16).)

Во втором случае проведем построение, аналогичное проделанному ранее в лемме 10.3. Именно, выберем в П $a$-ребро, которое не смежно в $\partial \Delta \mathrm{c} b$-ребром $e$, и максимально продолжим за него $a$-полосу $S=\left[\Pi, \Pi_{1}, \ldots, \Pi_{n}\right]$ (рис. 14 а с изменением обозначений). 
Допустив, что $S$ есть $a$-кольцо, получим противоречие с леммой 6.1(20). Допустив, что торец $e_{2}$ кольца $S$, отличный от торца $e_{1}$, смежного в $\partial \Delta$ с $e$, входит в $p_{X}$ (рис. $\left.14 б\right)$, получаем $(a, d)$-кольцо (так же, как возникло $\left(b, k_{1}\right)$-кольцо в лемме 10.3), что, однако, противоречит лемме 6.1(14).

Следовательно, торец $e_{2}$ полосы $S$ обязательно входит в путь $p_{Y}$.

Клетка П не может входить в $a$-полосу, начатую с другой $(d, a, b)$-клетки $\Pi^{\prime}$ (рис. 14в,г), имеющей $b$-ребро $e^{\prime}$ на $p_{X}$, так как это бы опять приводило к $(d, a)$-кольцу или к $a$-кольцу с $(d, a)$-клеткой (по аналогии с появлением $\left(b, k_{1}\right)$-кольца или $b$-кольца в лемме 10.3$)$, поскольку в $\Delta$ нет $d$-терминальных клеток. Поэтому построенные таким образом $a$-полосы $S$ и $S^{\prime}$ для разных $b$-ребер $e$ и $e^{\prime}$ пути $p_{X}$ имеют разные торцы $e_{2}$ и $e_{2}^{\prime}$ на пути $p_{Y}$.

Таким образом, закончено построение инъективного соответствия: $u$-ребрам пути $p_{X}$ сопоставлены $u$-ребра пути $p_{Y}$, a $b$-ребрам пути $p_{X}$ сопоставлены $b$-ребра пути $p_{Y}$ в случае (1) или $a$-ребра пути $p_{Y}$ в случае (2), а именно, торцы построенных по ним $a$-полос $S$, входящие в путь $p_{Y}$.

\section{§11. Расположение $s$-полос}

Наименее тривиальньм при доказательстве теоремы 3 является переход от длин элементов подгруппы $A_{4}$ к их длинам в группе $B_{6}$. Исследуемые при этом $s$-полосы включают в себя $a$-полосы. Связанные с ними оценки опираются, в частности, на свойства овалов из $\S 3$, гиперболичность “оценочного" графа $\Gamma_{t}(\Delta)($ лемма 9.4$)$ и отсутствие $r$-огибаний (лемма 8.4).

Лемма 11.1. Пусть $S-$-плолоса в минимальной диаграмме $\Delta$ над группой $B_{6}$. Тогда ее торцы е $e_{1}$ и е е не могут одновременно лежать на границе одного диска D.

ДокАзАТЕльСтво. Рассуждая "от противного", обозначим через $\delta(S)$ максимальную поддиаграмму в $\Delta$, ограниченную медианой полосы $S$. Ее граница имеет вид $p_{1} p_{2}$, где $p_{1}-$ край полосы $S$, а путь $p_{2}$ входит в $\partial D$.

Предположим сначала, что в пути $p_{2}$ встречаются $t$ - или $k$-ребра. Из вида (7.3) граничной метки диска следует, что $t$-ребра чередуются в $p_{2}$ с $k$-ребрами. Поэтому в $\delta(S)$ есть диски, ибо в $p_{1}$ не может быть $t$ - или $k$-ребер, а $t$-полоса не может пересекаться с $k$-полосой. Рассмотрим граф̆ $\Gamma_{t}(\delta(S))$, который должен быть $(2 L-1)$-графом по лемме 9.4. По лемме 3.2 в нем найдется вершина $o$, из которой во вне, т.е. в диск $D$, выходит не меньше $2 L-4>2$ спиц, причем между ними нет других дисков диаграммы $\Delta$. Но $o=o_{D^{\prime}}$ для некоторого диска $D^{\prime}$, и мы получаем противоречие с леммой 9.4 , примененной уже к $\Delta$. Таким образом, путь $p_{2}$ не содержит $t$ - или $k$-ребер, а в поддиаграмме $\delta(S)$ нет дисков.

Допустим, что торцы $e_{1}$ и $e_{2}$ разделены в $p_{2} Q$-ребром, которое может быть лишь одно, как видно из (7.3) и определения $h$-специального слова $\Sigma$. Поскольку в $p_{1}$ нет $Q$-ребер, граница $\partial \delta(S)$ содержит ровно одно $Q$-ребро. Но такого быть не может ввиду отсутствия в $\delta(S) Q$-терминальных клеток.

Остается рассмотреть случай, когда $\varphi\left(p_{2}\right)$ - подслово слова $\left(X^{\#}\right)^{-1}$ или слова $Y^{-1}$, где $\Sigma \equiv h^{-1} X^{\#} q_{j} Y h$. Но здесь слова $X, Y$ положительны, как видно из определения диска. Поэтому ориентируемость плоскости не позволяет обоим торцам полосы $S$ лежать на $\partial D$. 
Назовем $s$-yчастком подпуть в границе диска, меткой которого является $s$-слово.

Лемма 11.2. Пусть $S_{1}, \ldots, S_{n}$ - максимальные s-полосы (возможсно, без клеток), ториы $e_{1}, \ldots e_{n}$ которых лежсат на одном s-участке диска $D$ минимальной диаграммы $\Delta$ над группой $B_{6}$ (или на одном подпути р границь $\partial \Delta$, не содерәсащем $Q-u$-ребер), а другие торцы $e_{1}^{\prime}, \ldots, e_{n}^{\prime}-$ на границах $(r, Q)$-клеток, входящих в одну $r$-полосу $S$ (рис. $15 \mathrm{a})$. Тогда $n \leqslant 8$.

ДокАЗАТЕльство. Для доказательства можно уменњшить полосу $S=$ $\left[\Pi_{1}, \ldots, \Pi_{l}\right]$, считая, что как клетка $\Pi_{1}$, так и клетка $\Pi_{l}$ являются терминальными $(r, Q)$-клетками для названных $s$-полос, скажем для $S_{1}$ и $S_{2}$. Поскольку в границе каждой $(r, Q)$-клетки не больше четырех $s$-ребер, достаточно опровергнуть предположение, что в полосе $S$ имеется $(r, Q)$-клетка $\Pi=\Pi_{j}$, где $j \neq 1, l$. Покажем, что такое допущение ведет к появлению некоторого $(r, Q)$-кольца в поддиаграмме без дисков вопреки лемме 6.1(3).

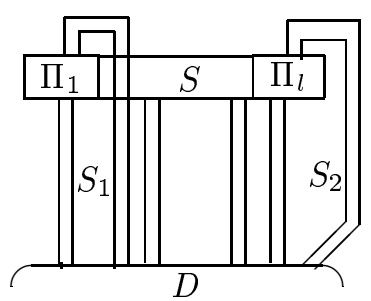

a

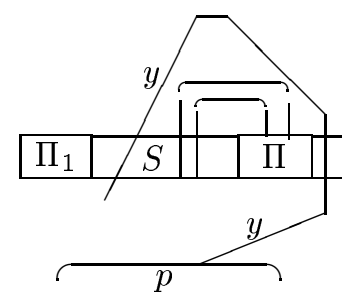

б

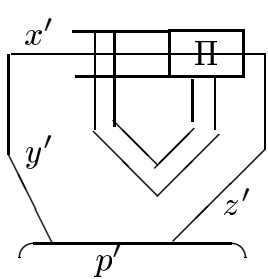

B

Рис. 15

Рассмотрим любую простую замкнутую кривую $c=y^{\prime} x^{\prime} z^{\prime} p^{\prime}$, составленную из частей медиан $y, z$ полос $S_{1}, S_{2}$, части средней линии $x$ полосы $S$ и части $s$-участка $p$ диска $D$. (Таких кривых можно составить несколько, если средние линии полос имеют по несколько пересечений.) Если в максимальной поддиаграмме $\delta(c)$, лежащей внутри $c$, есть диски, то по леммам 9.4 и $3.2 r$-полоса $S$ должна огибать некоторьй диск, ибо $2 L-4>L+1$, а в $S_{1}, S_{2}$ нет $t$-клеток и в $p$ нет $t$-ребер. Но это противоречит лемме 8.4 .

Напомним, что $(r, Q)$-клетка П не может входить в $s$-полосы. Допустим, что линия $y$ пересекает среднюю линию $x$ по разные стороны от клетки П (рис. $15 б)$. Тогда начатая с клетки П $Q$-полоса непременно пересечет еше раз $r$-полосу $S$, так как ее средняя линия не может пересечь линию $y$. Появляется $(r, Q)$-кольцо.

Следовательно, можно составить простую замкнутую кривую $y^{\prime} x^{\prime} z^{\prime} p^{\prime}$ такую, что ее $x$-часть $x^{\prime}$ проходит через клетку П (рис. 15в). Но тогда, как и выше, клетка П оказывается угловой в некотором $(r, Q)$-кольце, так как средняя линия $Q$-полосы не может пересекаться с дугами $y, z$ или $p$.

Лемма доказана.

Для произвольного слова $W$ обозначим через $|W|_{s}$ и $|W|_{r}$ соответственно число вхождений букв $s_{0}^{ \pm 1}, \ldots, s_{m}^{ \pm 1}$ и число вхождений букв $r_{i}^{ \pm 1}, i \in I$, в $W$. Положим также по определению

$$
|W|_{s, r}=|W|_{s}+60|W|_{r} .
$$


Если в минимальной диаграмме $\Delta$ над группой $B_{6}$ имеются диски, то граф $\Gamma_{t}(\Delta)$ является по лемме $9.4(2 L-1)$-графом. Рассмотрим некоторьй овал $p$, проведенный через вершину $o_{D}$, лежашую внутри диска $D$. Напомним, что в 33 было показано на основании леммы 3.5 , что в овал входит ровно два внешних ребра и он, являясь простой замкнутой кривой, определяет область $O(p)$ на плоскости. Внешние ребра, т.е. $t$-спицы из $p$, высекают из границы $\partial \Delta$ некоторый подпуть $\bar{p}=\bar{p}(D, p)$ целиком лежащий внутри области $O(p)$. (В него не входят два $t$-ребра диаграммы $\Delta$, пересекаемые внешними ребрами-спицами овала.) Назовем путь $\bar{p}$ тенъю диска $D$ на $\partial \Delta$, определенной овалом $p$.

По определению диска его граничной меткой является левая часть равенства (7.3) для некоторого слова $\Sigma(D)=\Sigma \equiv h^{-1} X^{\#} q_{j} Y h$ (или $\Sigma \equiv q$ ), где $\Sigma^{*}=q$ в полугрупше $\Gamma(T)$. Подпути контура диска $D$ с меткой $\Sigma^{ \pm 1}$ назовем $\Sigma$-дугами. Ключевой в этом параграфе является

Лемма 11.3. Пусть $W$ - слово, написанное на тени $\bar{p}$ диска $D$ минимальной диаграммы $\Delta$ над группой $B_{6}$. Тогда для слова $\Sigma=\Sigma(D)$ справедливо неравенство: $3|\Sigma|_{s, r}=3|\Sigma|_{s} \leqslant|W|_{s, r}$.

ДоКАЗАТЕЛЬСТво проведем с помощью индукции по числу вершин графа $\Gamma_{t}(\Delta)$, расположенных в определенной овалом $p$ области $O(p)$.

Поскольку $2 L-1 \geqslant 29$, в область $O(p)$ выходит из $D$ по определению овала не менее $\frac{1}{2}(2 L-3)+4$ спиц, т.е. в $O(p)$ находится не менее $2 \times 19=38$ последовательных $\Sigma$-дуг диска $D$. Отбрасывая по крайней мере по 4 первых и последних в этом ряду, обозначим 30 средних из них $p_{1}, \ldots, p_{30}$. Обозначим $\Delta(p)$ поддиаграмму, составленную из клеток, пересекаемых овалом $p$, а также целиком лежащих в $O(p)$. Отметим, что по лемме 9.5 в $\Delta$ нет $r$-колец, и рассмотрим далее два случая.

(1) Число $N_{r}$ максимальных $r$-полос в диаграмме $\Delta(p)$ не меньше $\frac{1}{20}|\Sigma|_{s}$.

Заметим, что хотя бы один торец каждой из этих полос должен лежать в тени $\bar{p}$. Действительно, в противном случае к графу $\Gamma=\Gamma_{t}(\Delta)$ и к медиане $x$ такой полосы можно применить лемму 3.7 , которая предоставляет либо $(r, t)$-кольцо в $\Delta$ без дисков внутри него вопреки лемме 6.1(5), либо огибание $r$-полосой некоторого диска вопреки лемме 8.4 .

Следовательно, $|W|_{s, r} \geqslant 60|W|_{r} \geqslant 60 N_{r} \geqslant 3|\Sigma|_{s}$.

(2) $N_{r}<\frac{1}{20}|\Sigma|_{s}$

Рассмотрим систему $\mathscr{S}$ максимальных $s$-полос с торцами на $\Sigma$-дугах $p_{1}, \ldots, p_{30}$. Отметим, что они не могут пересекать $t$-спицы, т.е. все они содержатся в $\Delta(p)$. Для полос, начинающихся на одной $\Sigma$-дуге $p_{i}$, не более $8 \times 2$ могут иметь торцы на $(r, Q)$-клетках одной $r$-полосы по лемме 11.2. Значит, на каждой из $r$-полос заканчивается не более $30 \times 16=480$ полос системы $\mathscr{S}$, а на всех $r$-полосах меньше $\frac{480}{20}|\Sigma|_{s}=24|\Sigma|_{s}$ полос ввиду условия (2). В дополнительной системе $\mathscr{T}$ содержится не менее $(30-24)|\Sigma|_{s}$ полос из $\mathscr{S}$, так как по лемме 11.1 вторые торцы полос из $\mathscr{S}$ не могут находится на $\partial D$.

Рассмотрим две полосы $S_{1}, S_{2}$ системы $\mathscr{T}$, заканчивающиеся на одном диске $D^{\prime}$ (если такие есть). В ограниченной их медианами области не может быть дисков, ибо по лемме 3.2 , примененной к соответствующему графу, вершина $о$ внутри одного из таких дисков давала бы 2-угольник в $\Gamma_{t}(\Delta)$ вместе с $o_{D}$ или $o_{D^{\prime}}$. Поэтому полосы $S_{1}$ и $S_{2}$ стартуют с $\Sigma$-дуг (и кончаются на $\Sigma^{\prime}$-дугах), разделенных не более 
чем одним ребром графа $\Gamma_{t}(\Delta)$, т.е. не более чем двумя $t$-ребрами на $\partial D\left(\partial D^{\prime}\right)$. (Напомним, что одна из $t_{1}$-спиц диска $D$ может быть стерта в графе $\Gamma_{t}(\Delta)$.) Это (и неравенство $2 L-1 \geqslant 29$ ) позволяет с помощью медианы $x$ любой полосы системы $\mathscr{T}$ между $D$ и $D^{\prime}$ определить один и тот же производный овал $p^{\prime}$, проходящий через $o_{D^{\prime}}$ (рис. $\left.16 \mathrm{a}\right)$.

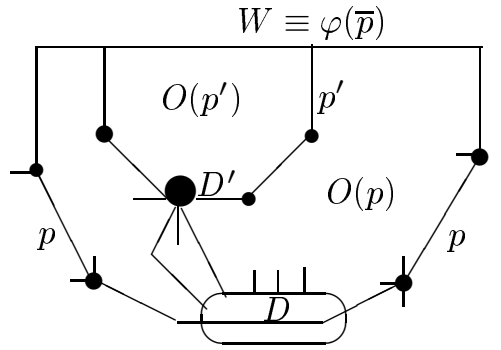

a

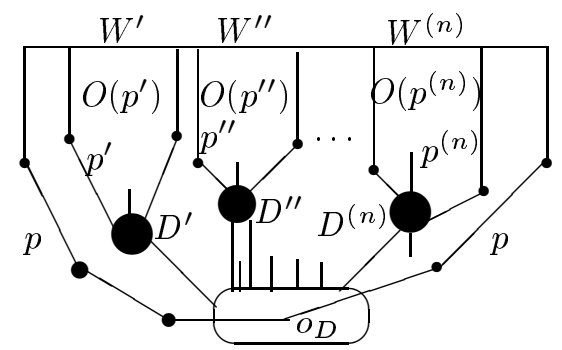

б

Рис. 16

Пусть $p^{\prime}, \ldots, p^{(n)}$ - всевозможные производные овалы, построенные для спиц подсистемы $\mathscr{T}_{1}$ системы $\mathscr{T}$, заканчивающихся на каких-либо дисках $D^{\prime}, \ldots, D^{(n)}$ (рис. 16б). Из леммы 3.8 следует, что тени этих дисков не пересекаются и находятся в тени диска $D$. Терминальными ребрами для полос из $\mathscr{T}_{2}=\mathscr{T} \backslash \mathscr{T}_{1}$ могут быть только ребра из тени $\bar{p}$, не входящие в тени перечисленных дисков. Поэтому ввиду линейности соотношения (11.1) для меток $W^{\prime}, \ldots, W^{(n)}$ теней этих дисков справедливо неравенство

$$
\left|W^{\prime}\right|_{s, r}+\cdots+\left|W^{(n)}\right|_{s, r}+\operatorname{card} \mathscr{T}_{2} \leqslant|W|_{s, r} .
$$

По той же лемме каждая область $O\left(p^{\prime}\right), \ldots, O\left(p^{(n)}\right)$ содержит меньше вершин графа $\Gamma_{t}(\Delta)$, чем $O(p)$. По индуктивному предположению

$$
3\left|\Sigma^{(i)}\right|_{s} \leqslant\left|W^{(i)}\right|_{s, r}
$$

для $i=1, \ldots, n$ и слов $\Sigma^{(i)}=\Sigma(D(i))$. В то же время

$$
6\left(\left|\Sigma^{\prime}\right|_{s}+\cdots+\left|\Sigma^{(n)}\right|_{s}\right) \geqslant \operatorname{card} \mathscr{T}_{1} \geqslant 6|\Sigma|_{s}-\operatorname{card} \mathscr{T}_{2},
$$

ибо разные полосы из $\mathscr{T}_{1}$ имеют торцы не более чем на шести $\Sigma$-дугах каждого из дисков $D^{\prime}, \ldots, D^{(n)}$. Из неравенств (11.2)-(11.4) следует, что

$$
3|\Sigma|_{s} \leqslant \sum_{i=1}^{n} 3\left|\Sigma^{(i)}\right|_{s}+\frac{1}{2} \operatorname{card} \mathscr{T}_{2} \leqslant \sum_{i=1}^{n}\left|W^{(i)}\right|_{s, r}+\frac{1}{2} \operatorname{card} \mathscr{T}_{2} \leqslant|W|_{s, r} .
$$




\section{§12. Доказательства теорем}

Докажем сначала возможность квазигеодезического вложения в теореме 3 , т.е. неравенство (12.1) для $u$-слов $U$. (Напомним, что группу $R$ можно отождествить с подгруппой $R_{u}$ групшы $B_{6}$ по определению группы $R_{u}$ и лемме 10.1.)

Итак, пусть произвольный элемент $g$ подгруппы $R_{u}$ представлен $u$-словом $U$ длины $\|U\|=|U|_{u}=|g|_{u_{1}, \ldots, u_{m}}$. Для произвольного слова $Z$ в порождаюших групшы $B_{6}$ обозначим $|Z|_{u, b, s, r}=|Z|_{u, b, s}+60|Z|_{r}$. Мы покажем, что если слово $Z$ представляет тот же элемент $g$, то

$$
\|U\|=|U|_{u} \leqslant|Z|_{u, b, s, r} \leqslant 60\|Z\| .
$$

Для этой цели зафиксируем какое-нибудь несократимое слово $Y$ в порождающих подгрупшы $A_{4}$ (см. $\left.\S 10\right)$, диаграмма $\Delta$ равенства которого слову $Z$ в группе $B_{6}$ имеет минимальный возможный тип. Такое слово $Y$ существует, так как $U=Z$ в $B_{6}$. Аналогично, для слова $Y$ существуют слова $X, W$ и $V$, удовлетворяющие условиям лемм $10.5,10.4$ и 10.3 соответственно. На основании лемм 10.2-10.5 имеем:

$$
|U|_{u} \leqslant|V|_{u} \leqslant|W|_{u, b} \leqslant|X|_{u, b} \leqslant|Y|_{u, b, a} .
$$

Поэтому для обоснования неравенства (12.1) нужна

ЛЕмма 12.1. Справедливо неравенство: $|Y|_{u, b, a} \leqslant|Z|_{u, b, s, r}$.

ДокАЗАтЕльство. Запишем контур диаграммы $\Delta$ в виде $p_{Y} p_{Z}^{-1}$, где $\varphi(Y) \equiv Y$, $\varphi(Z) \equiv Z$. Как и в леммах $10.2-10.5$, из выбора слова $Y$ следует, что путь $p_{Y}$ простой.

Граница никакой $u$ - или $b$-клетки диаграммы $\Delta$ не может иметь общее ребро c $p_{Y}$, так как граничные метки таких клеток записаны в порождающих подгруппы $A_{4}$, и, отрезая их от $\Delta$ с заменой $Y$ на $Y^{\prime}$, можно было бы уменьшить тип диаграммы. Значит, каждое $u$ - или $b$-ребро пути $p_{Y}$ входит и в путь $p_{Z}$. Поэтому для доказательства леммы достаточно установить неравенство $|Y|_{a} \leqslant|Z|_{s, r}$.

По той же причине, что и выше, никакое ребро пути $p_{Y}$ не может лежать на границе $(\sigma, a)-,(b, a)-,(a, d)$-, или $(a, r, x)$-клеток. Значит, для $a$-ребер пути $p_{Y}$ есть три возможности: принадлежать пути $p_{Z}$, принадлежать границе некоторой $(r, Q)$-клетки или принадлежать границе некоторого диска диаграммы. Первую возможность можем исключить: выбрасывая $a$-ребра первого типа, мы разрежем диаграмму $\Delta$ на несколько поддиаграмм, для каждой из которых утверждение леммы можно доказьвать отдельно, так как мы будем использовать лишш минимальность типа диаграммы равенства $Y=Z$.

В диаграмме $\Delta$ нет $r$-колец по лемме 9.5. Рассмотрим два случая.

(1) Число $N_{r}$ максимальных $r$-полос в $\Delta$ не менше $\frac{1}{60}|Y|_{a}$.

Допустим, что оба торца некоторой $r$-полосы $S$ находятся на пути $p_{Y}$. Тогда в максимальной поддиаграмме $\delta(S)$, расположенной в области, ограниченной медианой этой полосы и путем $p_{Y}$, нет дисков, как видно из сопоставления лемм 9.4, 3.2 и 8.4 , ибо путь $p_{Y}$ не содержит $t$-ребер. Более того, в $S$ нет $t$ - или $k$-клеток при $k \neq k_{1}$, так как в этом случае возникли бы $(r, t)$ - или $(r, k)$-кольца вопреки лемме $6.1(5,6)$. Аналогично, в $S$ нет $Q$-клеток по лемме $6.1(3)$, ибо в пути $p_{Y}$ нет 
$Q$-ребер. В таком случае метки клеток полосы $S$ - это слова в порождающих группы $A_{4}$. Поэтому слово $Y$ можно заменить словом $Y^{\prime}$, отрезая от $\Delta$ полосу $S$ вместе с поддиаграммой $\delta(S)$. Но такое уменьшение типа диаграммы противоречит выбору слова $Y$.

Таким образом, в пути $p_{Z}$ не меньше чем $N_{r} r$-ребер, откуда $|Z|_{s, r} \geqslant 60|Z|_{r} \geqslant$ $60 N_{r} \geqslant|Y|_{a}$.

(2) $N_{r}<\frac{1}{60}|Y|_{a}$

В этом случае из леммы 11.2 выводим, что не более 8 -ребер пути $p_{Y}$ могут принадлежать клеткам одной $r$-полосы, а общее число $a$-ребер пути $p_{Y}$, лежащих на границах $(r, Q)$-клеток, меньше $\frac{1}{7}|Y|_{a}$.

Пусть $\mathscr{T}$ - множество $a$-ребер пути $p_{Y}$, входящих в границы некоторых дисков. Мы установили уже, что сard $\mathscr{T}>\frac{6}{7}|Y|_{a}$.

Если некоторые ребра множества $\mathscr{T}$ входят в границу одного диска $D$, то они не могут быть разделены в $\partial D t$-ребрами. Для обоснования нужно рассмотреть поддиаграмму $\delta(D)$, заключенную между $D$ и путем $p_{Y}$. В ней нет дисков по леммам $9.4,3.2$, так как в пути $p_{Y}$ нет $t$-ребер. Поэтому начатая с $t$-ребра диска $D$ $t$-полоса не могла бы закончиться.

Таким образом, $a$-ребра пути $p_{Y}$ входят не более чем в два $\Sigma$-участка диска $D$. Поэтому (и ввиду неравенства $2 L-1 \geqslant 29$ ) для диска $D$ можно построить овал $p$ в графе $\Gamma_{t}(\Delta)$ такой, что никакое лежащее на диске $a$-ребро пути $p_{Y}$ не попадает в область $O(p)$, определенную этим овалом. В таком случае заданная овалом $p$ тень диска $D$ содержится в пути $p_{Z}$, поскольку в пути $p_{Y}$ нет $t$-ребер. Для метки $Z_{p}$ этой тени по лемме 11.3 справедливо неравенство

$$
\left|Z_{p}\right|_{s, r} \geqslant 3|\Sigma|_{s} \geqslant 3|\Sigma|_{a} \geqslant \frac{3}{2} n_{D}
$$

где $n_{D}$ - число $a$-ребер границы диска $D$, лежащих на пути $p_{Y}$.

Неравенство $2 L-1 \geqslant 29$ позволяет выбрать овал $p$ так, что любая $Г$-правильная дуга $x$ с концом на обшем $a$-ребре для $\partial D$ и $p_{Y}$ является 2-отделенной от овала $p$. Тогда овалы $p_{1}$ и $p_{2}$, построенные таким образом для двух дисков $D_{1}$ и $D_{2}$, имеюших общие граничные ребра $e_{1}$ и $e_{2}$ с $p_{Y}$, будут расходяшимися, поскольку точки на $e_{1}$ и $e_{2}$ всегда можно соединить подходящей $Г$-правильной дугой $x$. (Напомним, что эти ребра лежат на одном пути $p_{Y}$ без $t$-ребер.)

По лемме 3.6 овалы $p_{1}$ и $p_{2}$ не пересекаются во внутренних точках графа $\Gamma_{t}(\Delta)$. Поскольку дуга $x$ находится вне областей $O\left(p_{1}\right), O\left(p_{2}\right)$, эти области также не пересекаются. Значит, не пересекаются и тени дисков $D_{1}$ и $D_{2}$. Поэтому можно просуммировать неравенства вида (12.2) по всем дискам, имеющим общие ребра границы с путем $p_{Y}$ и получить:

$$
|Z|_{s, r} \geqslant \frac{3}{2} \sum n_{D} \geqslant \frac{3}{2} \frac{6}{7}\left|p_{Y}\right|_{a} \geqslant|Y|_{a}
$$

что и требовалось.

Если группа $G$ и функция $l$ заданы в теореме 2 , то сначала группу $G$ можно вложить в рекурсивно определяемую группу $R$ по лемме 2.2 и теореме 1 , так что функция $g \mapsto|g|_{R}$ эквивалентна функции $l$. Доказанное неравенство (12.1) и лемма 10.1 
означает эквивалентность функций длин в произвольной рекурсивно определенной конечно порожденной группе $R$ и построенной по ней большей группе $B_{6}$, которая может быть задана конечным множеством соотношений по лемме 4.7. Полагая $H=B_{6}$, завершаем доказательство теоремы 2 .

Чтобы перейти от неравенства (12.1) к равенству в теореме 3 , можно увеличить конечную систему порождающих подгруппы $R_{u}$, включив в нее, например, все элементы длины $\leqslant 60$ от порождающих $u_{1}, \ldots, u_{m}$. М. В. Сапир предложил автору другой прием.

Именно, введем новые буквы $r_{i j}$, где $i \in I, j=1, \ldots, 60$, и зададим группу $H$ теми же соотношениями, которьми определена группа $B_{6}$ в $\S 4$, но с заменой каждого из порождаюших $r_{i}$ во всех соотношениях на $\prod_{j=1}^{60} r_{i j}$. Из определения ясно, что корректно определен гомоморфизм $\psi: B_{6} \rightarrow H$, тождественный на всех порождающих кроме $r_{i}$, а $\psi\left(r_{i}\right)=\prod_{j=1}^{60} r_{i j}$. Левым обратным для него будет каждый из гомоморфизмов $\varepsilon_{j}, j=1, \ldots, 60$, тождественный на всех порождающих кроме $r_{i k}$, а $\varepsilon_{j}\left(r_{i j}\right)=r_{i}$ и $\varepsilon_{j}\left(r_{i k}\right)=1$ при $k \neq j$. Поэтому $\psi$ является изоморфным вложением групшы $B_{6}$ в $H$.

Очевидно, что для каждого элемента $g$ группы $R_{u}$ его длина относительно выделенной системы порождающих $\mathscr{B}$ группы $H$ не превосходит длины в порождающих $u_{1}, \ldots, u_{m}$, ибо $u_{1}, \ldots, u_{m} \in \mathscr{B}$. Допустим, что наоборот, $\left|Z_{0}\right|_{\mathscr{B}}<|U|_{u}$ для некоторого кратчайшего слова $U$, представляющего элемент $g \in R_{u} \leqslant H$, и некоторого слова $Z_{0}$ над алфавитом $\mathscr{B}$, представляющего тот же элемент. Выберем тогда такой индекс $j$, что обшее число вхождений в $Z_{0}$ всех букв из множества $\mathscr{R}_{j}=\left\{r_{i j}\right\}_{i \in I}$ не превосходит $\frac{1}{60}$ доли вхождений всех $r$-букв в слово $Z_{0}$. Очевидно тогда, что

$$
\left|Z_{0}\right|_{u}+\left|Z_{0}\right|_{b}+\left|Z_{0}\right|_{s}+60\left|Z_{0}\right|_{j} \leqslant\left\|Z_{0}\right\|<\|U\|
$$

где $\left|Z_{0}\right|_{j}$ - число $r_{i j}$-букв в $Z_{0}, i \in I$. Но тогда, применяя гомоморфизм $\varepsilon_{j}$, мы получим для образа $Z$ слова $Z_{0}$ равенство $U=Z$ в группе $B_{6}$, а из неравенства (12.3) - неравенство $|Z|_{u, b, s, r}<\|U\|$ вопреки неравенству (12.1).

Итак, построенное изоморфное вложение группы $R_{u} \cong R$ в конечноопределенную группу $H$ сохраняет длины элементов, и теорема 3 полностью доказана.

Для доказательства теоремы 4 достаточно сформулированное в ней свойство обеспечить для всех конечно-определенных групп $H$ ввиду теоремы 3 . Конечно-определенные группы вместе с выделенными в них непересекаюшимися системами порождающих можно эффективно перенумеровать: $\left(H_{1}, \mathscr{A}_{1}\right),\left(H_{2}, \mathscr{A}_{2}\right), \ldots$ Пусть $F$ - их свободное произведение с системой порождающих $\mathscr{A}=\left\{a_{1}, a_{2}, \ldots\right\}$, где $\mathscr{A}=\bigcup_{i=1}^{\infty} \mathscr{A}_{i}$. Определим группу $K$, добавляя к естественному копредставлению группы $F$ три порождающих $x, y, z$ и соотношения

$$
z^{-1} x^{-i} y x^{i} z=x^{-i} y x^{i} a_{i}, \quad i=1,2, \ldots
$$

Очевидно, что $K$ - конечно-порожденная рекурсивно определимая группа. Ее с сохранением длин можно вложить по теореме 3 в конечно-определенную группу $U$. Естественные гомоморфизмы групп $H_{j}$ в $K$ инъективны, ибо $K$ является в силу 
соотношений (12.4) HNN-расширением с базой $F *\langle x\rangle *\langle y\rangle$ и сопряженньми с помощью $z$ свободными подгруппами. Поэтому для доказательства теоремы 4 достаточно проверить, что длина любого элемента из $H_{j}$ в порождающих $\mathscr{A}_{j}$ не превосходит его длины в порождающих $x, y, z$.

С этой целью рассмотрим некоторое слово $W$ в порождающих $x, y, z$, представляющее некоторый элемент из подгруппы $H_{j}$ в $K$. Пусть $V$ - слово в порождающих системы $\mathscr{A}_{j}$ такое, что диаграмма $\Delta$ равенства $W=V$ имеет минимально возможное число клеток, отвечающих соотношениям группы $F$ и соотношениям (12.4). Достаточно показать, что $\|V\| \leqslant\|W\|$.

Граница диаграммы $\Delta$ имеет вид $p_{V} p_{W}^{-1}$, где $\varphi\left(p_{V}\right) \equiv V, \varphi\left(p_{W}\right) \equiv W$. При этом, как и в лемме 10.3 , путь $p_{V}$ простой и не имеет общих ребер с $a$-клетками, отвечающим соотношениям группы $H_{j}$. Как и в лемме 10.3 , каждое его ребро либо входит также в путь $p_{W}$, либо для него строится $z$-полоса из клеток типа (12.4) с торцом на пути $p_{W}$. Отсюда получается нужное неравенство $\|V\| \leqslant\|W\|$. Однако для полной аналогии с доказательством леммы 10.3 нужно объяснить еще, почему в минимальной диаграмме нет $z$ - и $(y, z)$-колец.

Последние свойства следуют из стандартных аргументов леммы 6.1. В частности, из них вытекает (см. доказательства свойств (9) или (11) в лемме 6.1), что гипотетическое $(y, z)$-кольцо должно состоять только из двух угловых клеток. Эти клетки дают сократимую пару, так как если они отвечали бы разным индексам $i$ из списка (12.4), то ограниченная медианой $(y, z)$-кольца поддиаграмма дала бы единичное в $K$ слово с ненулевой по $x$ суммой показателей. Но такое слово неединично даже в ретракте $\langle x\rangle$ группы $K$.

Теорема 4 доказана.

\section{Список литературы}

1. Olshanskii A. Yu. Distortion functions for subgroups // Proc. Conf. on Geometric Group Theory, Canberra, July 1996: Walter de Gruyter, 1997 (to appear).

2. Gromov M. Asymptotic invariants of infinite groups. Geometric group theory, № 2 // London Math. Soc. Lecture Note Ser. 1993. V. 182. P. 1-125.

3. Gersten $S$. Dehn functions and $l_{1}$-norms of finite presentations // Algorithms and computations in combinatorial group theory / ed. G. Baumslag, C. Miller. № 23: MSRI Publ., Springer, 1993.

4. Bridson $M$. Fractional isoperimetric inequalities and subgroup distortion // Preprint, 1996.

5. Birget J.-C. Time complexity of the word problem for semigroups and the Higman embedding theorem // LITP 95/26, Institut Blaise Pascal-4, Place Jusseu - 75252 Paris Cedex 05 .

6. Rotman J. An introduction to the theory of groups. 3rd ed: Allyn \& Bacon, 1984.

7. Aanderaa S. A proof of Higman embedding theorem using Britton extentions of groups // In Word problems, decision problems and the Burnside problem in group theory. Amsterdam: North-Holland, 1973. P. 1-18.

8. Birget J.-C., Rips E., Sapir M. V. Dehn functions of groups // Preprint, 1997.

9. Линдон Р. и IIynп П. Комбинаторная теория групп. М.: Мир, 1980.

10. Ольшанский А. Ю. Геометрия определяющих соотношений в группах. М.: Наука, 1989.

Московский государственнњй

Поступила в редакцию

университет им. М.В. Ломоносова

01.04 .1997 